\title{
BIOPHYSICAL OVERVIEW, SIGNIFICANT, SENSITIVE AND DISTURBANCE FEATURES \\ OF THE EAGLE BUTTE SENSITIVE AREA
}

\author{
Prepared for: \\ Resource Data Division \\ Alberta Environmental Protection \\ Edmonton, Alberta
}

Prepared by:

D.A. Bradshaw, A. Saxena and I.D. Macdonald

GEOWEST Environmental Consultants Ltd.

Bedford Square

Suite 203, 4209 - 99 Street

Edmonton, Alberta T6E 5 V7

March, 1996 
Digitized by the Internet Archive in 2017 with funding from University of Alberta Libraries

https://archive.org/details/biophysicaloverv1996brad 


\section{EXECUTIVE SUMMARY}

The Eagle Butte Sensitive Area is one of eight sensitive areas identified in southern Alberta by Alberta Fish and Wildlife Services. It is located in the southeastern corner of Alberta and encompasses approximately $326 \mathrm{~km}^{2}$ of native vegetation as well as dissected and slumping slopes of the Cypress Hills, supporting a mosaic of grassland and forest vegetation types. It includes portions of Township 7 - Ranges 3 and 4, and Township 8 - Ranges 1 through 4, all W4M. Cypress Hills Provincial Park is located immediately adjacent the study area. The hamlet of Elkwater is located immediately outside the study area, along the south shore of Elkwater Lake. The hamlet of Manyberries is situated approximately $13 \mathrm{~km}$ to the southwest. Northern portions of the Eagle Butte Sensitive Area extend to the Alberta-Saskatchewan border. The Canada - United States border is approximately $58 \mathrm{~km}$ south of the study area. The Manyberries Badlands Sensitive Area is located approximately 3 kilometres to the southwest.

Increased petroleum exploration activity in southeastern Alberta and the resulting surface disturbances have put pressure on sensitive areas within the region. GEOWEST Environmental Consultants Ltd. was contracted to undertake a study, commissioned by Fish and Wildlife Services, Lethbridge, to identify the vegetation communities as well as significant, sensitive and disturbance features present in the Eagle Butte Sensitive Area. The intent of the study is to serve as an initial source for use by the petroleum industry, and which will help identify areas where detailed site-specific study may be necessary. Specific objectives of the project were:

- to undertake a field inventory and assessment of the Eagle Butte Sensitive Area using existing and new information, and to produce a report and five thematic maps for the following: 
- vegetation communities

- significant features

- disturbance features
- sensitive features

- slope classes

Thirteen native vegetation community types were identified, with classification based on landscape attributes which influence vegetation community development. One other type encompassing cultivated areas was also mapped. The Native Grassland community type is the most extensive in the area, with communities associated with the eroding slopes to the Cypress Hills Plateau and with hummocky moraine areas also comprising a significant part of the study area. The study area encompasses the flanks of the Cypress Hills, with the vegetation in these areas reflecting the relatively greater moisture regime found there. Native grassland communities are transitional between mixed grass prairie at lower elevations and rough fescue grassland at higher elevations. Forests dominated by aspen, lodgepole pine and white spruce occur at higher elevations, extending into grassland areas of lower elevations via sheltered, north-facing drainage valleys. These forest communities share more similarities with western Cordilleran forests of southwestern Alberta than with boreal forests of central and northern Alberta.

The Eagle Butte Sensitive Area contains a proportionately greater number of rare plant and animal species than many other areas in the surrounding Mixed Grassland Natural Subregion. Northern leopard frog (Rana pipiens), burrowing owl (Athene cunicularia), Baird's sparrow (Ammodramus bairdii) and swift fox (Vulpes velox) are among the notable fauna which occur within the study area. The study area also comprises a major component of key areas for elk (Cervus elaphus), moose (Alces alces), mule deer (Odocoileus hemionus) and white-tailed deer (O. virginianus). The Eagle Butte Sensitive Area may contain up to 21 species of rare or uncommon plants or species with restricted distribution in Alberta, plus three species representing range extensions. Of these, 14 species confirmed in the study area by GEOWEST personnel and previous researchers. Such species include marsh butterweed 
(Senecio foetidus), rare in Canada, and tall meadow rue (Thalictrum dasycarpum), American pellitory (Parietaria pensylvanica), pine-drops (Pterospora andromedea) to name a few, all rare in Alberta.

The Eagle Butte Sensitive Area possesses some relatively unique physical and topographic features, including the flanks of the Cypress Hills and parts of the unglaciated Cypress Hills Plateau. The area is geologically diverse, with five bedrock formations exposed in the study area. As well, numerous springs of good quality potable water discharge in the study area. Six significant areas were mappable in the study area, ranging from the flanks and plateau of the Cypress Hills within the study area, to riparian and floodplain areas within Medicine Lodge Coulee which provide critical summer and winter ranges for mule deer and whitetailed deer. Three of the six significant areas were rated as having national significance and one other was rated as provincially significant.

Slopes within the Eagle Butte Sensitive Area are highly variable and consist of both simple and complex slopes, with complex slopes predominant. The Eagle Butte Sensitive Area predominantly consists of extensive areas with strong to rugged topography. Much of the upland areas in western portions of the study area are bedrock controlled, exhibiting rolling to ridged topography with complex slopes usually spanning a wide range of slope classes. The eroding and slumping slopes common on the flanks of the Cypress Hills, Medicine Lodge Coulee, Manyberries Creek valley and the many tributary drainages of these areas consist of complex slopes ranging from moderate to steep. The Eroding Slopes and Shrub Draw community types possess the strongest relief in the study area, with slopes generally ranging from extreme to steep, with some eroding slope areas having very steep slopes (greater than 100 percent slope). Complex slopes are also prevalent within the Hummocky Grassland community type in the northern portion of the study area. Slopes here range from moderate to strong, becoming relatively more subdued in northeastern portions of the study area, with slopes ranging from gentle to moderate. In addition, the Riparian community type possesses 
complex slopes due to the many small fans, terraces and meander scars found within these areas. Slope gradient and complexity has implications with regards to vegetation community establishment in terms of their effects on moisture regimes and on the intensity of aspect effects. Both of these factors affect, in part, the type of vegetation community found at a given site. Implications also exist with regards to the potential for erosion within a given unit, which is a factor influencing the inherent sensitivity of an area to disturbance.

The Eagle Butte Sensitive Area, as the name suggests, is considered sensitive as a whole, due to physical and climatic attributes of the area. A significant portion of the Eagle Butte Sensitive Area has been classed as having very high or high sensitivity to disturbance. Community types often corresponding to these areas include the Eroding Slopes, Shrub Draw and forested community types. These are areas of complex slopes with strong relief. Erosional effects subsequent to physical disturbance are expected to be severe, and reclamation success is anticipated to be low. Numerous springs are located in these areas, and which provide potable water and support rare plant populations in many cases. The Eroding Slopes community type may also provide habitat for rare flora and fauna. Northern leopard frog habitat areas are also highly sensitive to physical disturbance such as those arising from oil and gas activity or wetland drainage.

Cattle grazing on native prairie is one of the primary land uses of the area. Large scale disturbances have been caused by the active cultivation of land and the creation of tame or "improved" pasture, encompassing approximately 12 percent of the entire study area. Cultivation for the most part has been limited to lands with simple slopes of low relief, as found on the Cypress Hills Plateau in the southeastern portion of the study area, within the valley bottoms of Medicine Lodge Coulee and Manyberries Creek valley, and on localized relatively level areas scattered through the remainder of the study area. Other disturbance features within the study area include wellsites, clay and gravel quarries, water developments such as reservoirs and dugouts, and linear disturbances such as from gravel roads, trails and 
pipelines. These disturbances are largely concentrated in the central and southern portions of the study area.

This study will be best used as an initial source by both industry and government agencies to "flag" potential areas of concern related to any proposed development activity such as for oil and gas exploration and development. Detailed field study may often be necessary to confirm site-specific occurrences of significant flora and fauna within the study area. 


\section{ACKNOWLEDGEMENTS}

GEOWEST would like to acknowledge the contributions of the following people in bringing this project to completion.

GEOWEST Environmental Consultants Ltd. personnel involved in the project included Don Bradshaw as principal author and editor. Wildlife information was compiled and synthesized by Amit Saxena. Field work for the project was undertaken by lan D. Macdonald, Amit Saxena and Veronica Chisholm. Preliminary interpretation was by Jerry Bentz. John Sisson and Della Clish prepared final maps and figures, and Terry Lang provided clerical support.

GEOWEST would like to thank Keith Ainsley of Resource Information Division, Alberta Environmental Protection, for project coordination. As well, Dean Wetzel and Mark Woodhouse of Alberta Community Development, Cultural Facilities and Historical Resources Division, Resource Management Programs Unit, Edmonton, provided valuable input concerning the archaeological and historical resources of the study area. 


\section{TABLE OF CONTENTS}

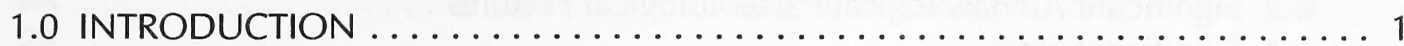

2.0 STUDY AREA DESCRIPTION $\ldots \ldots \ldots \ldots \ldots \ldots \ldots \ldots \ldots \ldots \ldots \ldots \ldots \ldots \ldots \ldots \ldots$

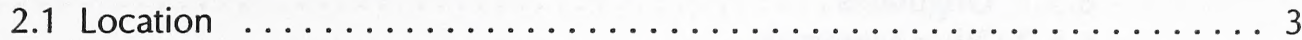

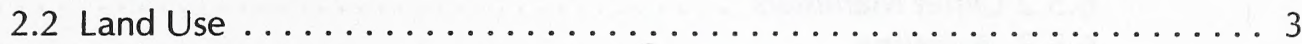

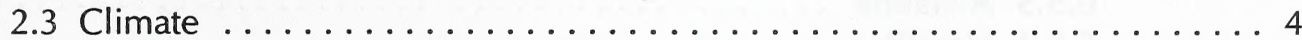

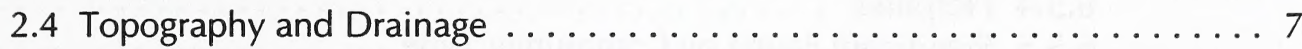

2.5 Bedrock Geology . . . . . . . . . . . . . . . . . . . . 9

2.6 Surficial Geology ............................ 11

2.7 Soils . . . . . . . . . . . . . . . . . 12

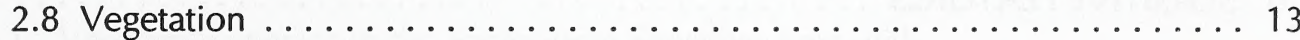

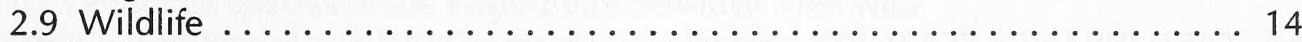

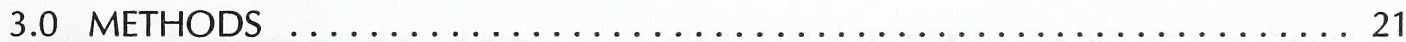

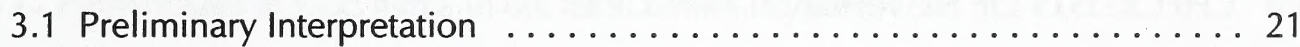

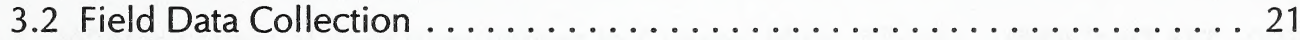

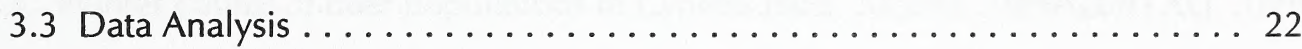

4.0 VEGETATION COMMUNITY TYPES ..................... 26

4.1 Native Grassland . . . . . . . . . . . . . . . . . . . 28

4.2 Native Grass/Shrub Complex. . . . . . . . . . . . . . . . . . . . 34

4.3 Coulee Slopes. . . . . . . . . . . . . . . . . . . . 37

4.4 Eroding Slopes. ............................... 44

4.5 Alluvial Fans/Floodplains . . . . . . . . . . . . . . . . 50

4.6 Hummocky Grassland ... . . . . . . . . . . . . . . . . . . . . 54

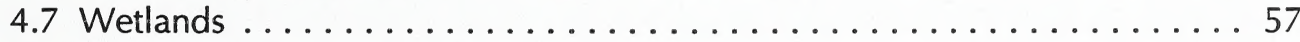

4.7.1 Permanent Wetland ........................ 57

4.7.2 Ephemeral Wetland ...................... 59

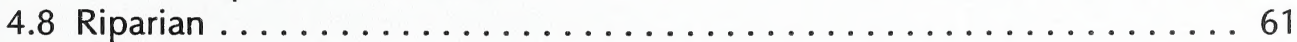

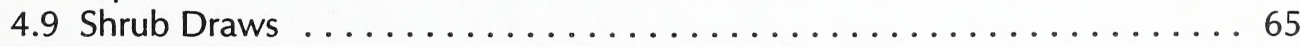

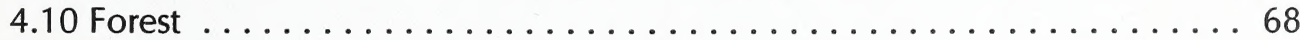

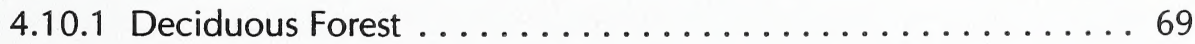

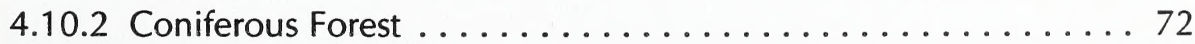

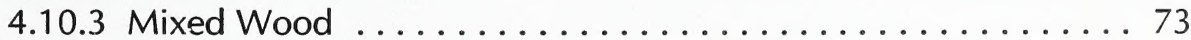

4.11 Agricultural . . . . . . . . . . . . . . 76

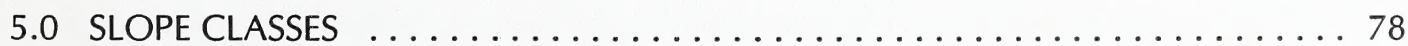




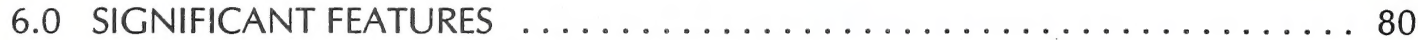

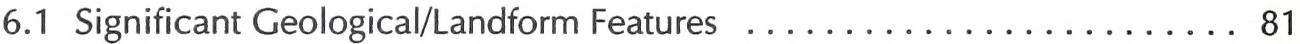

6.2 Significant Hydrological Features . . . . . . . . . . . . . . . . 82

6.3 Significant Archaeological/Palaeotological Features . . . . . . . . . . . 84

6.4 Significant Flora . . . . . . . . . . . . . . . . . . . 85

6.5 Significant Fauna . . . . . . . . . . . . . . . . . . 86

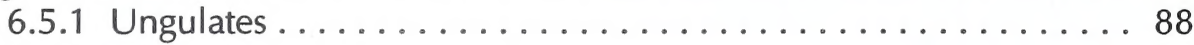

6.5.2 Other Mammals ....................... 95

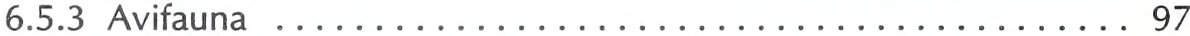

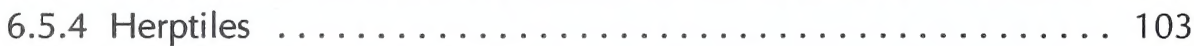

6.5.5 Significant Fauna by Community Type . . . . . . . . . . . 105

6.6 Significant Areas . . . . . . . . . . . . . . . . . . 113

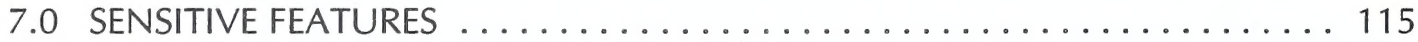

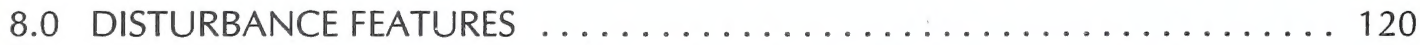

9.0 CHECKLISTS OF SIGNIFICANT FEATURES $\ldots \ldots \ldots \ldots \ldots \ldots \ldots \ldots \ldots \ldots$

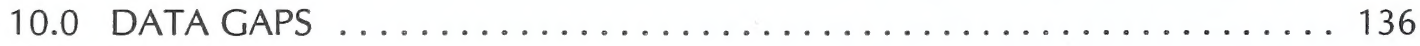

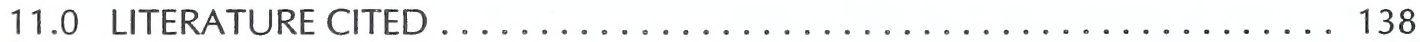

PERSONAL COMMUNICATIONS ........................ 148

APPENDIX A - Significant Floral Features of the Eagle Butte Sensitive Area

APPENDIX B - Plates 


\section{LIST OF FIGURES}

Figure 1. Location of the Eagle Butte Sensitive Area ............. 6

\section{LIST OF TABLES}

Table 1. Vertebrate species in the Eagle Butte Sensitive Area with affinities to Boreal and Cordilleran faunal elements ............. 18

Table 2. Percentages of food items in elk fecal pellets, Cypress Hills . . . . . . . . . 90

Table 3. Winter counts of deer populations in Cypress Hills, Alberta, 1971-1991 . . . 93

Table 4. Significant species of forest-dwelling birds in the Cypress Hills . . . . . . 101

Table 5. Significant fauna and habitat use by vegetation community type . . . . . 112 


\subsection{INTRODUCTION}

The settlement of the Canadian prairies and the subsequent cultivation of grasslands for agricultural purposes has resulted in very few areas of native prairie remaining in Canada which are contiguous and relatively large. The Eagle Butte Sensitive Area is located within one such area.

The Eagle Butte Sensitive Area is one of a number of sensitive areas identified as providing critical or important habitat for various threatened and endangered wildlife species, such as northern leopard frog (Rana pipiens) and Baird's sparrow (Ammodramus bairdii). The study area also provides important winter and summer range for elk (Cervus elaphus), moose (Alces alces), mule deer (Odocoileus hemionus) and white-tailed deer (Odocoileus virginianus). In addition, the Eagle Butte Sensitive Area also contains plant species which are rare in Alberta or rare in Canada.

In recognition of the fragmentation and development pressures facing native prairie ecosystems in Canada, initiatives such as the Prairie Conservation Action Plan were developed to influence policies and attitudes concerning native prairie in order to conserve the biological diversity of native prairie ecosystems (World Wildlife Fund Canada 1988). Provincial government agencies are currently working to implement the goals of the Prairie Conservation Action Plan in the management of public land and resources in Alberta (Anonymous 1992, Public Lands Division 1990).

Increased petroleum exploration activity in southeastern Alberta and the resulting surface disturbances have put pressure on sensitive areas within the region. In some cases, exploration companies are required to prepare a land surface management plan to ensure that adverse effects in sensitive areas are minimized. Plan development requires the identification of significant and sensitive physical and biological features which may be affected by the 
proposed development, with mitigative measures then proposed to minimize adverse effects to these features from petroleum development activities.

The purpose of this study, as commissioned by Fish and Wildlife Services, Lethbridge, was to use existing and new information to identify the vegetation communities as well as significant, sensitive and disturbance features present in the Eagle Butte Sensitive Area. The intent is for the study to serve as an initial source for use by industry as land surface management plans are developed, and which will help identify areas where detailed sitespecific study and other investigations may be necessary. As well, it is anticipated that government agencies may also use this report as an initial source during the course of referrals concerning the study area, with the report serving as an aid to "flag" areas of potential concern to the particular agency as related to any proposed land disposition.

The specific objectives of this project were:

- to undertake a field inventory and assessment of the Eagle Butte Sensitive Area, and - to produce a report and five thematic maps for the following:

- vegetation communities

- significant features

- disturbance features
- sensitive features

- slope classes

The results of this study provide a source for both industry and government agencies to identify potential areas of concern related to any proposed development activity such as oil and gas exploration and development. Detailed field study may often be necessary to confirm site-specific occurrences of significant flora and fauna within the study area. 


\subsection{STUDY AREA DESCRIPTION}

\subsection{Location}

The Eagle Butte Sensitive Area is found in the southeastern corner of Alberta and encompasses approximately $326 \mathrm{~km}^{2}$ of native vegetation as well as dissected and slumping slopes of the Cypress Hills, supporting a mosaic of grassland and forest vegetation types. It includes portions of Township 7 - Ranges 3 and 4, and Township 8 - Ranges 1 through 4, all W4M. (Figure 1). Cypress Hills Provincial Park is located immediately adjacent the study area. The hamlet of Elkwater is located immediately outside the study area, along the south shore of Elkwater Lake. The hamlet of Manyberries is situated approximately $13 \mathrm{~km}$ to the southwest. Northern portions of the Eagle Butte Sensitive Area extend to the AlbertaSaskatchewan border. The Canada - United States border is approximately $58 \mathrm{~km}$ south of the study area. The Manyberries Badlands Sensitive Area is very near, located approximately 3 kilometres to the southwest.

\subsection{Land Use}

Slightly more than half of the study area is titled land. The remainder is public land, most of which is under grazing lease. The study area predominantly consists of native prairie utilized for cattle production, though cultivation is somewhat frequent, concentrated largely in relatively level areas. Petroleum development is minor in the study area. Quarrying for clay and gravel is relatively common in the study area.

A recreational lease on public land exists for NE 21 and SE 28 - Twp. 7 - Rge. 3 - W4M. This lease land is currently under consideration as a candidate Natural Area.

Cypress Hills Provincial Park is located immediately adjacent to the study area. 


\subsection{Climate}

The climate of the prairies of southeastern Alberta is characterized by long, hot and dry summers and cold winters, with high evaporation rates as a result of strong winds and low humidity. The Cypress Hills, however, provide a unique "biological-climatological" environment due to an "oasis effect" of cooler and moister conditions resulting from the relatively rapid elevational change of the Cypress Hills Plateau above the surrounding prairie (Holmes 1970, 1969). Wind flow patterns over the Cypress Hills have a pronounced effect on moisture and vegetation distribution by creating areas of enhanced or reduced rainfall, drifted snow, evaporation enhancement and air drainage (Holmes 1970, 1969). Moisture deficits occur at lower elevations, promoting the distribution of grasslands. This moisture deficit is severe on south-facing slopes and particularly on southwest-facing slopes which are exposed to hot and dry prevailing winds. In contrast, a moisture surplus exists at higher elevations, particularly on sheltered, north-facing slopes, promoting the existence of forest communities in these areas (Breitung 1954).

In terms of the climatically-based ecoregion classification system of Strong (1992), the study area is transitional between the Mixed Grass Ecoregion and the Montane Ecoregion, with lower elevations of the study area classified within the Mixed Grass Ecoregion and higher elevations to the Cypress Hill Plateau classified within the Montane Ecoregion. Climate data is lacking for the Cypress Hills specifically. For the Mixed Grass Ecoregion in general, average annual precipitation totals $326 \mathrm{~mm}$, with approximately 66 percent of this $(214 \mathrm{~mm})$ occurring during the growing season of May through September (Strong 1992). June is the wettest month. Average annual temperature is $5.3^{\circ} \mathrm{C}$, with a monthly mean of $-6.3 \mathrm{C}$ in January and an average temperature of $14.4^{\circ} \mathrm{C}$ during the growing season of May through September. Mean growing degree days for the ecoregion are 1442.

In contrast, generalization of climate data for the Montane Ecoregion shows an average 
annual precipitation of $440 \mathrm{~mm}$, with approximately 59 percent of this $(259 \mathrm{~mm}$ ) occurring during May through September (Strong 1992). June and August are the wettest months. Average annual temperature is $3.5^{\circ} \mathrm{C}$, with a monthly mean of $-6.6 \mathrm{C}$ in January and an average temperature of $11.4^{\circ} \mathrm{C}$ during May through September. Mean growing degree days for the ecoregion are 773. 
Figure 1: Location of the Eagle Butte Sensitive Area

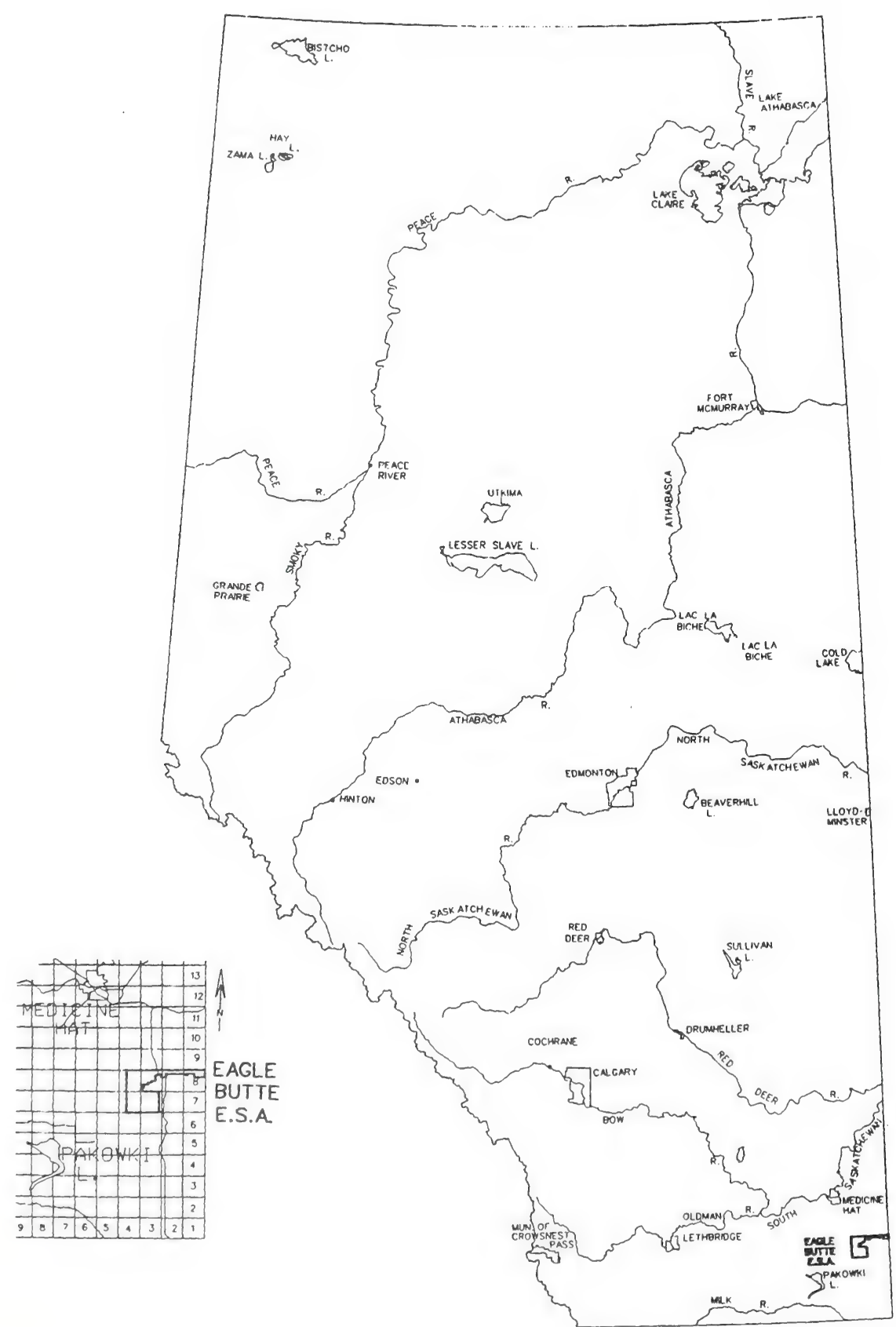




\subsection{Topography and Drainage}

The study area was part of a preglacial drainage divide which extended from the Cypress Hills to the Sweet Grass Hills (Westgate 1968). The Eagle Butte Sensitive Area now straddles three different drainage systems. Northern portions of the study area are within the South Saskatchewan drainage system, which eventually empties into Hudson Bay. Bullshead Creek is part of this system (Westgate 1968). However, much of the surface water flow north from the Cypress Hills is restricted to internal drainage systems of intermittent streams and alkaline lakes, with relatively little of the water reaching the South Saskatchewan River (Newsome and Dix 1968).

Southwestern portions of the study area drained by Manyberries Creek form part of an internal drainage system centred on Pakowki Lake. The southern portion of the study area is part of the Milk River drainage basin which, in turn is part of the Missouri River drainage system which drains into the Gulf of Mexico. Lodge Creek is part of this system.

Laurentide ice sheets covered the entire area during the Pleistocene epoch. The Elkwater advance was the furthest and the most recent, extending approximately to the present-day location of Great Falls, Montana (Westgate 1968). Within the study area, the Elkwater drift is the surface drift limited to the immediate vicinity of the Cypress Hills. This drift is thin and discontinuous, consisting of sporadic patches of glacial gravel and tills with some large erratics.

The Wild Horse advance was the second-most recent advance, and the Wild Horse drift comprises the surface drift in southern portions of the study area and around the study area perimeter. This drift is generally less than 50 feet thick. Till of the Wild Horse drift is of a clay-loam to loam texture. Glacier flow was influenced by local topography in southeastern Alberta, and the Laurentide ice sheets were forced to the southeast between the Cypress Hills and Sweet Grass Hills. 
The current topography of the area is a result of glacial and postglacial deposition and erosion (Westgate 1968). Topography is highly variable and complex within the Eagle Butte Sensitive Area, depending on the glacial ice features which deposited the parent material, the thickness of parent material, and on subsequent, post-glacial erosional and depositional processes. Topography ranges from undulating to ridged over large portions of upland areas in western and southern portions of the study area, largely controlled by underlying bedrock with till veneers, to hummocky over much of the northern portion of the area below the escarpment of the Cypress Hills. Eroded coulees and dissected topography is extensive in the study area as a result of glacial meltwater channels and post-glacial erosional processes which have exposed the underlying bedrock (Shetsen 1987, Westgate 1968).

As well, slumping is extensive within the study area, particularly within Medicine Lodge Coulee and its tributaries, as well as along northern and western escarpments of the Cypress Hills. This is perhaps the prevalent erosional process currently affecting the study area. This process is attributed to the percolation of precipitation through the porous sand and gravels of the conglomerate of the Cypress Hills Formation. The resulting groundwater, in addition to emerging as numerous springs along valley slopes, also weakens the subsurface clays prevalent along the flanks of the hills, causing extensive slumping of these slopes (Roed n.d.).

Elevations within the study area generally increase from the outer perimeter of the study area towards the Cypress Hills, with the elevational increase becoming rapid approaching the Cypress Hills. The Cypress Hills in general decrease in elevation from western portions in Alberta to eastern portions in Saskatchewan. The median elevation of the study area is approximately 1269 metres above sea level (masl), with elevations ranging from approximately 1082 mas! in the northwest corner of the study area on hummocky moraine, to approximately 1455 masl in central portions of the study area immediately south of Cypress Hills Provincial Park. This represents a significant elevational relief of approximately 373 metres over a relatively short linear distance. 
Groundwater flow is away from the Cypress Hills, flowing generally toward the north, with glacial drift serving as the main aquifer (Borneuf 1976). Thalwegs, or buried bedrock channels, which comprise branches of the preglacial Medicine Hat bedrock channel exist under Bullshead Creek and Gros Ventre Creek, trending to the north. Also, a thalweg exists under Manyberries Creek, beginning approximately at the southern boundary of the study and trending to the southwest, eventually turning north and entering the Medicine Hat thalweg north of Pakowki Lake.

\subsection{Bedrock Geology}

The Cypress Hills are an erosional remnant capped by the Cypress Hills Formation, the youngest formation in the region, formed during the Tertiary Period and consisting of a conglomerate of pebbles and cobbles within a matrix of calcareous sandstone, with minor sandstone lenses (Borneuf 1976). The Cypress Hills area formed part of a preglacial drainage divide extending from the Cypress Hills to the Sweet Grass Hills (Westgate 1968). Medicine Lodge Coulee cuts across this drainage divide, exposing numerous bedrock formations within the study area along the flanks of the Cypress Hills and of Eagle Butte. Eagle Butte itself are believed to be remnants of the Flaxville Plain, gravel-capped and lithologically similar to the Cypress Hills but younger (Westgate 1968). The following formations are discussed in order of increasing age.

The Ravenscrag Formation is of Tertiary age, lying beneath the Cypress Hills Formation. This non-marine formation is exposed along the upper edge of the Cypress Hills escarpment, and consists of sandstones, clays, coal seams and bentonitic layers (Westgate 1968). Within the study area the formation occurs primarily in the northeastern third of Township 7 - Range 3 W4M, and also covers a small portion of the study area in the vicinity of Sections $33-36$, Twp. 8 - Rge 2 and Section 31 - Twp. 8 - Rge 1 - W4M (Borneuf 1976). 
Strata of the Frenchman Formation are also confined to the Cypress Hills. A few outliers of this formation occur in the study area in the vicinity of Eagle Butte and Fly Lake (Westgate 1968). The formation originated during the latter part of the Cretaceous Period and is composed of massive sandstones with some siltstones and shales.

The Battle Formation is a relatively thin formation consisting of uniform dark clays and bentonitic shales. This formation tends to occur in conjunction with the Whitemud Formation immediately below. Both formations are discontinuous, having been eroded away in places prior to the deposition of the Frenchman Formation (Westgate 1968).

The Whitemud Formation itself is a relatively thin formation, occurring on the north and south slopes of the Cypress Hills, with outcrops occurring in the study area primarily west of the Cypress Hills in the vicinity of Eagle Butte and Fly Lake (Westgate 1968). The formation consists of non-marine sandstones, white clay, kaolinised sandstones, carbonaceous shales and lignite. This formation is the best clay unit in the area, and is mined at clay quarries, such as Quarry No. 4 north of Fly Lake, to supply industrial ceramic plants in Medicine Hat (Roed n.d.). Several abandoned clay pits are located along the sides of Eagle Butte.

The sandstone strata of the Eastend Formation are situated below the Whitemud Formation. This thick, non-marine formation is confined to the Cypress Hills area, occurring in a belt at the foot of the Cypress Hills escarpment. The formation is dissected by Medicine Lodge Coulee, which has also downcut into the underlying Bearpaw Formation (Borneuf 1976).

The Bearpaw Formation is marine in origin and underlies an extensive region flanking the Cypress Hills (Borneuf 1976). The formation is essentially comprised of dark grey and brownish grey shale, with occasional thick sandstone beds. Spheroidal ironstone concretions commonly occur in the formation (Westgate 1968). 
A series of northwest- to southeast-aligned thrust faults extend into the extreme northwest corner of study area in the vicinity of Section 33-Twp. 8-Rge. 4-W4M (Westgate 1968). Bedrock beds in this area are highly disturbed, with shale of the Alberta Formation brought to the surface. The Alberta Formation is extremely old, dating to the Lower Cretaceous Epoch. Displacement of this formation to the surface may be due to the occurrence of an igneous body at depth, likely related to the igneous intrusions of the Sweet Grass Hills (Westgate 1968).

\subsection{Surficial Geology}

The Eagle Butte Sensitive Area is generally overlain by a thin mantle of glacial material, generally no more than 15 metres in thickness (Westgate 1968). However, those portions of the study area which are part of the Cypress Hills Plateau were unglaciated. These unglaciated areas largely consist of the eastern portion of Township 7 - Range 3 - W4M. Parent materials in these areas consist of loess deposits generally less than 1.5 metres thick.

Much of the remainder of the study area consists of thin till veneers over bedrock uplands. Such areas extend west and north of Medicine Lodge Coulee, and also extend in a relatively narrow band along the base of the north slopes of the Cypress Hills to the AlbertaSaskatchewan border (Westgate 1968).

Medicine Lodge Coulee is an ice-marginal meltwater channel. Ice-marginal channels are cut into glacial drift and often also cut into the underlying bedrock (Westgate 1968). This is the case for Medicine Lodge Coulee, which cuts across a preglacial drainage divide. Terraces of glacio-fluvial sand and gravel can be found within this coulee. As well, numerous small meltwater channels extend along the base of the Cypress Hill Plateau. The meltwater channels in the study area have served to sharply define the northern and western escarpments of the Cypress Hills (Beaty 1975). 
The northern margin of the study area consists of hummocky disintegration moraine, formed near the ice margin, creating a "knob-and-kettle" topography of weak to strong surface expression consisting of relatively low, circular or oval mounds with shallow central depressions (Westgate 1968). Minor areas of end moraine occur in the study area north of the Cypress Hills Plateau (Westgate 1968), manifested in the study area largely as aligned linear ridges.

Recent fluvial deposits can be found in Medicine Lodge Coulee and other drainages, deposited by present-day creeks. Lacustrine deposits occur within permanent and ephemeral wetlands in the study area.

\subsection{Soils}

The study area is transitional between the Dark Brown and Black soil zones which are manifested over a relatively short distance as elevation increases to the Cypress Hills Plateau. Dark Brown soils are common in those portions of the study area at lower elevations. With increased elevation and corresponding increases in precipitation and moisture regimes, Black soils become common.

Chernozemic soils are predominant within the study area (Wyatt et al. 1941, Greenlee 1981, Leskiw 1990). Lower elevations in this area are commonly Dark Brown Chernozems which become transitional to Black Chernozems as elevation increases to the Cypress Hills Plateau. Textures are predominantly loam, with some sandy loams and shallower soil profiles at higher elevations.

Soils within forested areas can be Chernozemic, exhibiting evidence that these soils developed under grassland vegetation, and include Orthic Dark Gray Chernozems. Orthic Gray and Dark Gray Luvisols are also possible. 
Humic Gleysols can be found on poorly drained sites. Orthic Regosols and Rego Gleysols can be found in riparian areas.

Solonetzic soils can be found primarily in southern portions of Medicine Lodge Coulee due to the influence of the marine Bearpaw Formation. Shallow, eroded pits, often referred to as "blowouts", are common where Solonetzic soils occur. The solonetzic B horizon can be exposed over extensive areas.

Orthic Regosols and Rego Chernozems can be expected on eroding slopes.

\subsection{Vegetation}

Native vegetation of the Eagle Butte Sensitive Area is highly variable, encompasses communities typical of Dark Brown soil zones of the Mixed Prairie (Coupland 1961, 1950), communities typical of rough fescue grasslands within the Black soil zone (Moss and Campbell 1947, Moss 1944, Coupland and Brayshaw 1953, Breitung 1954), and forested communities sharing many similarities with western cordilleran forests (Newsome and Dix 1968, Breitung 1954). The influence of regional climate and, most importantly in the Cypress Hills, local climate is reflected in the vegetation by which extensive grasslands predominate at lower elevations, with deciduous and coniferous forests occurring at higher elevations of the Cypress Hills. Tree and extensive shrub growth is also able to occur in moist, sheltered locations at lower elevations, such as spring-fed coulees and riparian areas. The study area straddles both the Mixedgrass Subregion of the Grassland Natural Region and the Montane Subregion of the Rocky Mountain Natural Region of Achuff (1994). Vegetation in the study area can therefore be expected to be transitional between the two ecoregions, as described above.

Prevalent grass species within the study area include western porcupine grass (Stipa curtiseta) 
June grass (Koeleria macrantha) and northern wheat grass (Agropyron dasystachyum) at lower elevations, and rough fescue (Festuca scabrella), California oat grass (Danthonia californica) and Hooker's oat grass (Helictotrichon hookeri) at higher elevations. Grassland communities transitional between Stipa-dominated and Festuca-dominated communities are common.

Prevalent tree species in forest communities in the study area include aspen (Populus tremuloides), lodgepole pine (Pinus contorta) and white spruce (Picea glauca).

The actual community and species representation at any given site will be influenced by numerous factors, including elevation and its effects on annual precipitation, as well as microsite variability in terms of aspect, slope, moisture regime and drainage, salinity and other soil properties, and grazing intensities.

Detailed discussion of the vegetation of the study area is presented in Sections 4.0 and 6.4 and Map 1.

\subsection{Wildlife}

The Eagle Butte Sensitive Area is characterized by a broad diversity of wildlife species, owing to its highly variable topography and resultantly variable microclimatic and vegetational conditions. Faunal species presence, abundance, and distribution is well documented for habitats in and around Cypress Hills Provincial Park (for example, Lombard North Group 1973, Lewis and Johnson 1980, Western Ecological Services 1980). The location of the Eagle Butte Sensitive Area on the slopes of the Cypress Hills represents a major source of both diversity and uniqueness among biotic communities of the Sensitive Area and allows close correlations to be drawn from the Cypress Hills faunal assemblage to those in the Eagle Butte Sensitive Area. 
The Cypress Hills are isolated erosional remnants that rise from 454 to 758 metres above the surrounding plain. Cooler temperatures and greater precipitation result in a lower rate of evaporation, thus allowing more moisture for plant growth and providing conditions which favor the establishment of forest vegetation. Resultantly, the vegetation, as described previously in Sections 2.8 and 4.0, is a mixture of prairie vegetation, characteristic of the surrounding plains, and montane vegetation, characteristic of the Rocky Mountain foothills. The moister, cooler climate of the elevated hills has created a diversity of characteristically montane habitats that are not found anywhere else in the dry mixed grassland region of Canada (Holmes 1969).

Many theories have been presented in attempts to explain the presence of montane habitats and wildlife species in the Cypress Hills and Eagle Butte areas. McCorquedale (1965) provided the following potential explanations for the isolated Cordilleran flora of the area:

(a) there was a forest connection between the mountains and the Cypress Hills area in early post-glacial times;

(b) some of the forest species that followed the retreating glacier remained in the Cypress Hills area; or,

(c) an interglacial flora of this area survived the Wisconsin glaciation because the ice did not override the higher portions of the plateau.

The latter premise that the elevated plateaus of the Cypress Hills escaped landscape and climatological changes associated with the glaciation, allowing pre-glacial life forms to persist in the hills is no longer deemed valid. Although it is true that a small part of the plateau was not covered by ice, the climate which existed there during glaciation would have been drastically affected by the presence of such a large ice mass and vascular plant life 
would likely not have survived (Downs 1981). However, in the period following glacial recession from this area, the climate was suitable for forest growth and a forested connection likely existed between the Rocky Mountain foothills and the Cypress Hills. Shifts to hotter and drier climates are reported by Downs (op cit) to have caused the destruction of this connection, except in areas where the climate retained enough rainfall and cool enough temperatures to support the existing forest vegetation. Due to its elevation, regions surrounding the Cypress Hills and Eagle Butte were such areas.

Native prairie wildlife habitats are widely acknowledged as representing some of the most severely threatened wildlife habitats in the arid and semi-arid regions of the world, due largely to human activity (Johnson et al. 1985, World Wildlife Fund Canada 1988, Epp 1991, Anonymous 1992). The present, post-glacial fauna of the Eagle Butte Sensitive Area, as with other prairie habitats in Canada, is characterized by an assemblage severely impacted by human habitation and use of the region. Risser (1988) recognized the most striking feature of the prairie in its historical state as being its vast array of native herbivores. Historic estimates of 60 million plains bison made this species the most significant herbivore on the plains, however its disappearance from the Cypress Hills by 1880 marked the species' extirpation from one of its last strongholds on the Canadian prairies. Excessive and uncontrolled hunting in the Cypress Hills and Eagle Butte area also contributed to the extirpation from the region of the plains grizzly bear (Ursus arctos) and the bobcat (Lynx americanus) by 1890 , elk by 1909, cougar (Felis concolor) by 1914, gray wolf (Canis lupus) by 1925 , and trumpeter swans by the 1950s (Ressler n.d., Halladay 1965, Scace 1978, Downs 1981, MacAuley 1995).

In the decades following the 1920s, human modification of the faunal assemblage of the Eagle Butte area turned from depletion and use to protection and introductions. The elk populations which had been extirpated from the area by 1909 were re-established through introduction efforts begun in 1938 in the Saskatchewan portion of the Cypress Hills (Lombard 
North Group 1973). Elk are now fairly abundant in the Eagle Butte area, with critical wintering ranges occurring both within Cypress Hills Provincial Park and within the Eagle Butte Sensitive Area. Non-native species have also since been introduced to the area, as ruffed grouse (Bonasa umbellus) were introduced to the Provincial Park area in 1922, ten red squirrels (Tamiasciurus hudsonicus) were introduced to the Saskatchewan side of the Cypress Hills in 1950, moose (Alces alces) were released on the Alberta side of the Cypress Hills in 1956, and Merriam's wild turkey (Meleagris gallopavo) were released in the park area in 1962 (Ecoplans Ltd. 1982, MacAuley 1995).

The significance of the present-day fauna of the Eagle Butte Sensitive Area is found in the fact that it is here where biota with a distinctly southern accent reaches northward across the 49th parallel. In stark contrast, the Eagle Butte Sensitive Area also houses biota which exhibit strong affinities to boreal and cordilleran faunal elements characteristic of northern Alberta and the Rocky Mountains, respectively. Species such as swift fox (Vulpes velox), Richardson's ground squirrel (Spermophillus richardsoni), bobolink (Dolichonyx oryzivorus), Baird's sparrow (Ammodramus bairdii), and upland sandpiper (Bartramia longicauda) are found in open grassland habitats which surround the forested plateau. Boreal and cordilleran faunal elements are represented in the Eagle Butte Sensitive Area by species such as longtailed vole (Microtus longicaudus), ermine (Mustela erminea), ring-necked duck (Aythya collaris), poor-will (Phalaenoptilus nuttallii), dusky flycatcher (Empidonax oberholseri), tree swallow (Tachycineta bicolor), and red-breasted nuthatch (Sitta canadensis). Overall, MacAuley (1995) identified 55 vertebrate species with affinities to northern Boreal and/or western Cordilleran habitats (Table 1). 


\section{Table 1: Vertebrate species in the Eagle Butte Sensitive Area with} affinities to Boreal and Cordilleran faunal elements

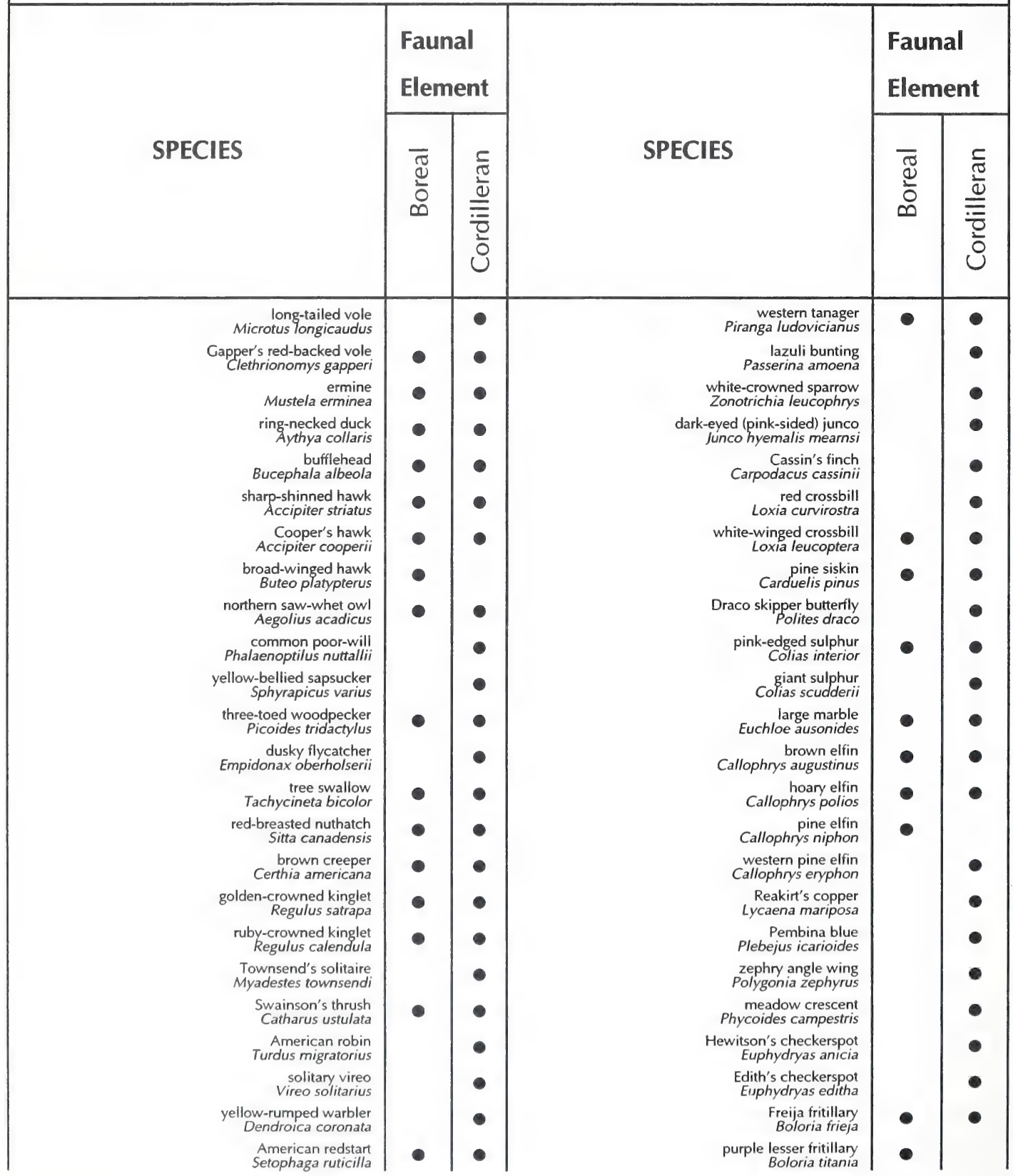




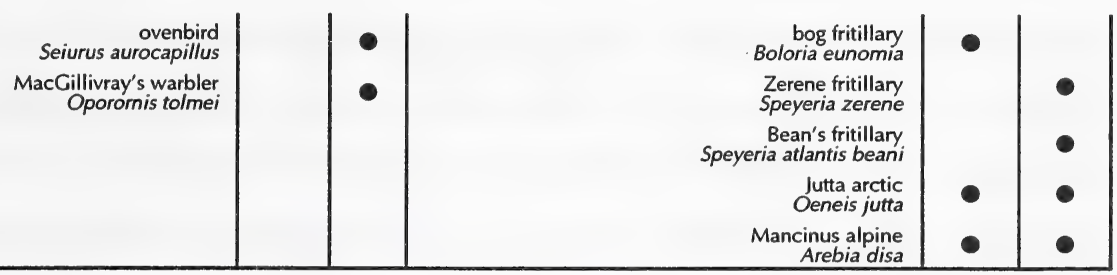

Lombard North Group (1973) reported 111 bird species in the Cypress Hills, while MacAuley (1995) reported sightings of over 200 species, 90 of which were thought to be nesting in the Cypress Hills area. Numerous bird species, including the house sparrow (Passer domesticus), the ring-necked pheasant (Phasianus colchicus), European starlings (Sturnus vulgaris), ruffed grouse, and Merriam's turkey, have been introduced to the area by early European settlers. Uncommon and/or threatened avian species such as the upland sandpiper, Baird's sparrow, and sharp-tailed grouse can also be found in appropriate habitats in the Eagle Butte Sensitive Area. The Mearn's, or pink-sided, race of the dark-eyed junco is common in the study area, but is not found anywhere else in Canada (Wallis and Wershler 1980).

Hunter (1990) recognized that the composition and structure of floral habitats is reflected by the distribution and abundance patterns of avifauna species. In addition, factors such as water, nutrients, sediments, and organic matter in vegetated riparian areas result in the development of extremely diverse, heterogenous, and productive biotic communities. Therefore, increased abundance and diversity of breeding birds can be witnessed in both riparian woodlands and in coniferous forests of the plateau lands.

Wetlands within the study area are areally limited, most occuring along the northern stretch of the study area, bordering Cypress Hills Provincial Park. Many of these wetlands, however, are ephemeral in nature and correlate with areas of relatively heavy snow accumulation during winter. As such, the capability of the study area to support significant populations of waterfowl and shorebirds is fairly minimal. Waterfowl are generally restricted to the larger, permanent lakes and reservoirs such as Michel Reservoir and Elkwater Lake. However, 
herpetofaunal species rely on wetland and aquatic habitats for critical life requisites and some wetlands in the study area may be disproportionately important compared to their size for such species. Beaver (Castor canadensis) pond habitats located along Sexton Creek provide abundant habitat for northern leopard frog (Rana pipiens), a species which has suffered a severe decline in its provincial range.

Four species of ungulates - mule deer (Odocoileus hemionus), white-tailed deer (Odocoileus virginianus), moose, and elk - regularly occur in the study area at varying levels of abundance. In concert with the large numbers of domestic livestock in the study area, these herbivores are the dominant faunal species in the study area, exerting influences on open grassland and riparian ecosystems. Use of habitats within the study area by these ungulates is largely seasonal and is dictated by availability of appropriate browse and forage. 


\subsection{METHODS}

\subsection{Preliminary Interpretation}

A series of 1992 1:30,000 black and white panchromatic (June 8,1992) aerial photographs were used to identify vegetation types, slope classes, special features and disturbance features, with the initial lines then transferred on to a digitally produced 1:20,000 base map.

An extensive literature review was also undertaken to determine special features previously identified in the study area and in adjacent areas. As well, a file review and examination of maps from the Cultural Facilities and Historical Resources Division, Resource Management Programs Unit office in Edmonton was completed.

Seven primary sources of information were obtained that assisted in the identification of vegetation community types. These included the Ecology of Mixed Prairie in Canada by Coupland (1950); A Reconsideration of Grassland Classification in the Northern Great Plains of North America by Coupland (1961); A botanical survey of the Cypress Hills by Breitung (1954); The forests of the Cypress Hills, Alberta and Saskatchewan, Canada by Newsome and Dix (1968); The fescue grassland of Alberta by Moss and Campbell (1947); The fescue grassland in Saskatchewan by Coupland and Brayshaw (1953); and A study of the ecology of grassland communities and the effect of grazing in the Cypress Hills Provincial Park, Alberta by Lewis and Johnson (1980). These reports identify a number of community types common to the Cypress Hills area.

\subsection{Field Data Collection}

Field work was undertaken during the summer of 1995, conducted during various periods between mid-August to the end of September. One hundred and fifty (150) sites were surveyed with data being recorded for slope, aspect, landform, percent exposed soil, 
ecological moisture regime, soil surface texture and plant species composition. Vegetation sampling consisted of a sample line 25 meters long and 0.5 meters wide situated within homogeneous stands, oriented across the slope. The sample line was subdivided into five contiguous blocks of 5 meters by 0.5 meters, creating five sampling rectangles of $2.5 \mathrm{~m}^{2}$ each. The percentage of herbaceous plant foliar cover was then visually estimated. Percent foliar cover of shrubs was visually estimated in five circular plots, each being 5 meters in diameter. Within forested communities, percentage foliar cover of tree species was visually estimated within two circular plots at each site, each with a radius of five meters. Vegetation data was summarized on Vegetation Description Form LISD 14B (Rev. 3/93). Site information was collected as per the Ecological land survey site description manual (Alberta Environmental Protection 1994) and recorded on Site Description Form LISD 15B (Rev. 3/93).

Pictures were also taken at each site to illustrate the physiographic and physiognomic characteristics. Notes were made regarding wildlife habitat and wildlife observations.

\subsection{Data Analysis}

Vegetation community classification for the Eagle Butte Sensitive Area was not done on the basis of plant species dominance in the community. To do so would imply a level of sampling intensity which allowed species dominance to be determined and mapped throughout the study area. This sampling intensity did not exist for this study. Vegetation community types were thus defined and delineated on the basis of landscape features and physiognomic characteristics mappable from aerial photography. Parameters used for the classification included slope, aspect, ecological moisture regime, nutrient regime, parent material, soil texture, topographic position, drainage and salinity. Vegetation patterns arising from the above parameters were identified and are described in this report. 
Slope classes follow those defined in the physical land classification methodology of Kocaoglu (1990). Mapping conventions are summarized within the slope class map legend (Map 2) and will not be elaborated on here. Slope class polygons were delineated through aerial photograph interpretation, and checked during the course of field sampling.

The parameters which defined the slope classification were used in conjunction with the consideration of additional edaphic and biotic factors to determine and map classes of sensitivity to physical disturbance. Additional edaphic factors considered included physiography, drainage and salinity, among others. Biotic factors included the actual or potential occurrence of important plant or animal habitats within the unit.

The identification of significant features, particularly of rare flora and fauna, relied heavily on review of existing information as time constraints precluded the undertaking of intensive surveys required to identify populations in the field. Extrapolation was often required of data from studies in areas adjacent to the Eagle Butte Sensitive Area. Detailed field study will be required in many cases to confirm the occurrence of significant flora and fauna specifically within the study area. The significance of sites within the Eagle Butte Sensitive Area was based on a consideration of 12 criteria as outlined by Eagles (1984), including:

- areas which perform a vital environmental, ecological or hydrological function such as aquifer recharge

- $\quad$ areas which contain rare or unique geological or physiographic features - $\quad$ areas which contain significant, rare or endangered plant or animal species

- $\quad$ areas which are unique habitats with limited representation in the region or are a small remnant of once large habitats which have virtually disappeared - $\quad$ areas which contain an unusual diversity of plant and/or animal communities due to a variety of geomorphological features and microclimatic effects 
- $\quad$ areas which contain large and relatively undisturbed habitats and provide sheltered habitat or species which are intolerant of human disturbance

- $\quad$ areas which provide an important linking function and permit the movement of wildlife over considerable distances, including migration corridors and migratory stopover points

- $\quad$ areas which contain plants, animals or landforms which are unusual or are of local, regional, provincial, national or international significance

- $\quad$ areas that are excellent representatives of one or more ecosystems, habitats or landscapes

- areas with intrinsic appeal due to widespread community interest or the presence of highly valued features or wildlife species valued for hunting

- $\quad$ areas which perform a vital function for wildlife in the area

- $\quad$ areas with cultural, historical or archaeological significance.

The significance of each site was categorized according to Eagles (1984) as follows:

International - features which are unique in the world

National - features which are limited in distribution at a national level or which are the best or only representatives in Canada

Provincial - features which are of limited distribution or are the best examples of a feature in the province

Regional - features which are of limited distribution or are the best examples of a feature in the region 
Local - features which are of limited distribution or are the best examples of a feature in the study area.

Disturbance features within the study area were identified from aerial photograph interpretation and field investigations. Three classes of roads were mapped: major allweather roads, including all gravelled secondary highways; minor gravelled roads; and vehicle trails on ungravelled mineral soil. Active oil and gas well sites were not distinguished from each other, but were simply mapped as "well site". Reclaimed well sites of either type were simply mapped as "reclaimed well site". Pipelines and flow lines were all mapped as "pipeline". Fencelines, usually consisting of barbed wire fences, were not delineated, as insufficient time was available for field checking to determine whether contrasts observed on aerial photography were the result of fencelines or of reverting native prairie subsequent to past cultivation - a common occurrence in the vicinity of the Cypress Hills. Other disturbance features identified in the study area included cultivated areas, water reservoirs and dugouts, quarries (mapped as "pit"), farm sites, drainage canals, a cemetery and a bible camp.

Polygons and map codes for all mapping themes were digitized using Intergraph Microstation software to produce .dgn files, which were then converted to .EOO files using ARC/INFO software.

Five thematic maps at 1:20 000 have been produced to accompany this report. These maps include:

- vegetation communities

- significant features

- disturbance features
- slope classes

- sensitive features 


\subsection{VEGETATION COMMUNITY TYPES}

In addition to the influence of annual precipitation, vegetation community development is influenced by microsite variability in terms of aspect, slope, moisture regime and drainage, salinity and other soil properties, and grazing intensities. The relatively rapid elevational change within Eagle Butte Sensitive Area due to its location on the flanks of the Cypress Hills influences many of the above parameters, resulting in a mosaic of mixed prairie grassland associations, rough fescue grasslands and coniferous and deciduous forest vegetation which is unique from the surrounding mixed grass prairie.

The "oasis" effect of the Cypress Hills on local climate, as described by Holmes $(1969,1970)$, creates a situation of higher moisture effectiveness resulting from lower surface temperatures and greater precipitation than the surrounding plains (Coupland 1961, Breitung 1954). Rough fescue (Festuca scabrella) dominated grasslands, described as the Festuca scabrella Association by Moss and Campbell (1947), Coupland and Brayshaw (1953), Coupland (1961) and Breitung (1954), occur at higher elevations, becoming prevalent at approximately 1200 meters above sea level. Mixed grass prairie (the Stipa-Agropyron Faciation of the Mixed Prairie association of Coupland (1961) or the Agropyron-Stipa Association of Breitung (1954)), comprise the grasslands at lower elevations. Grassland communities transitional between rough fescue grasslands and mixed grass prairie occur at intermediate elevations.

A close relationship also is evident between vegetation and topography (Breitung 1954), with site characteristics such as slope, aspect and subsequent effects on ecological moisture regime reflected, among other factors, in the plant communities occurring at a given site. For example, grassland communities typical of subxeric to xeric sites in southeastern Alberta occur in the study area where such conditions exist, such as on slopes with southern aspects. Conversely, mesic conditions as found on north-facing slopes, for example, support woody vegetation ranging from shrub to forest communities. 
The numerous springs which discharge in the study area along the flanks of the Cypress Hills also influence vegetation patterns, supporting plant communities adapted to these moist conditions.

A detailed, intensive vegetation inventory was beyond the purview of this study. As such, the identification of vegetation communities based on plant species names, such as a Stipa comata - Bouteloua gracilis community type, was not considered appropriate as this would imply a level of inventory or sampling intensity which did not exist for this study. Vegetation community types were thus defined and delineated on the basis of landscape features and physiognomic characteristics which are mappable from aerial photography.

Fourteen community types were identified and delineated as a result of aerial photo interpretation and field work, and are outlined as follows:

1. Native Grassland

2. Native Grass/Shrub Complex

3. Coulee Slopes

4. Eroding Slopes

5. Alluvial Fans/Floodplains

6. Hummocky Grassland

7. Wetland
a. Permanent Wetland
b. Ephemeral Wetland

8. Riparian

9. Shrub Draws

10. Forested
a. Deciduous Forest
b. Coniferous Forest
c. Mixed Wood

11. Agricultural

The Native Grassland community type was, in areal terms, the most extensive type within the study area. 
Similar community types have previously been described by Coupland (1961, 1950), Coupland and Brayshaw (1953), Moss (1944), Moss and Campbell (1947), Breitung (1954), Newsome and Dix (1968), Lewis and Johnson (1980) and Bradshaw et al. (1995, 1994). Vegetation community types of the Eagle Butte Sensitive Area are presented in Map 1.

The vegetation communities identified above are described below with reference to plant species composition, topographic position and site characteristics.

\subsection{Native Grassland}

The Native Grassland community type occurs on well drained upland areas within the study area. Topography in the unit is highly variable, ranging from rolling to ridged at bedrock controlled areas at lower elevations, to gently undulating with gentle (2 - $9 \%$ slopes) on the Cypress Hills Plateau. Parent materials vary within the unit, being predominantly morainal in origin, but with occurrences of glacio-fluvial and eolian veneers and blankets as well. Soils are predominantly Chernozems, ranging from Dark Brown at lower elevations to Black at higher elevations (Greenlee 1981, Leskiw 1990, Wyatt et al. 1941). Soil textures are commonly sandy loam to sandy clay loam. Ecological moisture regime ranges from subxeric to xeric at lower elevations, to mesic to subxeric at higher elevations. Nutrient regime generally is mesotrophic.

Grasses are the dominant plants in this community type. The Native Grassland community type fundamentally consists of two different vegetation associations, one typical of mixed grass prairie (specifically, the Stipa-Agropyron Faciation of the Mixed Prairie association of Coupland (1961) or the Agropyron-Stipa Association of Breitung (1954)), and the Festuca scabrella Association as described by Coupland (1961), Coupland and Brayshaw (1953), Moss and Campbell (1947) and Breitung (1954). Inventory sampling intensity was insufficient to differentiate these plant associations during mapping, however, the general 
pattern of occurrence of the plant associations within the Native Grassland community type are described below.

Mixed Prairie vegetation typical of the Stipa-Agropyron Faciation of the Mixed Prairie association of Coupland (1961) (the Agropyron-Stipa Association of Breitung (1954)), occurs on Dark Brown soils in the study area, primarily at lower elevations. Ecological moisture regimes are subxeric or drier. Western needle grass (Stipa curtiseta) is commonly the predominant grass, with June grass (Koeleria macrantha) usually comprising an important proportion of the community to the point where Lewis and Johnson (1980) named the grassland community on similar sites as the Stipa-Koeleria community type. However, June grass is an increaser under grazing within Mixed Prairie communities, and the prevalence of June grass may likely be an expected response given the grazing history of the area. Other grasses commonly present include northern wheat grass (Agropyron dasystachyum), blunt sedge (Carex obtusata), low sedge (Carex stenophylla), rough hair grass (Agrostis scabra), thread-leaved sedge (Carex filifolia) and needle-and-thread (Stipa comata). The prevalence of these species at a given site will vary according topographic position and associated effects on moisture regime, as well as from the effects of grazing intensities. Western needle grass is predominant on excellent condition range. Western needle grass decreases under higher grazing intensities, with June grass increasing and prairie selaginella (Selaginella densa) becoming more prominent.

As elevations increase and as moisture regimes increase, rough fescue grassland communities typical of the Festuca scabrella Association described by Coupland (1961) and others (see above) occur on Black, predominantly Chernozem, soils. This association generally becomes widespread in the Cypress Hills at approximately 1220 meters above sea level and higher (Breitung 1954). Ecological moisture regime at these sites ranges from submesic to mesic. Rough fescue grassland communities in the study area tend to represent disclimax stages of the Festuca scabrella Association, where California oat grass (Danthonia californica) 
comprises an important component of the stand at some sites, as well as communities transitional between rough fescue-dominated communities and western needle grassdominated communities of lower elevations. Western needle grass becomes abundant as moisture regimes become drier as a result of elevation or physiographic factors such as aspect and slope (Coupland 1961). These transitional communities are the most common communities found in the Native Grassland community type within the study area.

Plant species often occurring along with rough fescue and which reflect mesic moisture regimes include slender wheat grass (Agropyron trachycaulum), mat muhly (Muhlenbergia richardsonis), Hooker's oat grass (Helictotrichon hookeri), northern bedstraw (Galium boreale), golden bean (Thermopsis rhombifolia) and silvery perennial lupine (Lupinus argenteus). As well, shrubby cinquefoil (Potentilla fruticosa) can occur as a frequent shrub within rough fescue communities. Shrubby cinquefoil is characteristic of the Festuca scabrella Association in the Cypress Hills and differentiates the association within the Cypress Hills from the Festuca scabrella Association where it occurs in aspen groveland areas in eastcentral portions of Alberta (Coupland and Brayshaw 1953).

At sites subject to prolonged, heavier grazing pressure, rough fescue decreases markedly and plant species which increase to comprise an important proportion of the community include California oat grass, low sedge (Carex stenophylla), June grass and, under prolonged heavy grazing pressure, Kentucky bluegrass (Poa pratensis).

Shrubs occur as occasional on xeric sites, or relatively frequent, with patchy distribution on sites reflecting a relatively greater moisture regime such as in minor depressions and draws, or north-facing slopes. On xeric sites, shrubs such as Nuttall's atriplex (Atriplex nuttallii) are occasional. Prairie rose (Rosa arkansana) and common wild rose (R. woodsii) can occur as occasional on open grassland areas. On moister sites as described above, shrub patches commonly consist of buckbrush (Symphoricarpos occidentalis) or various species of rose. 
The Native Grassland community type is the most common type on upland areas and on open plateau areas. The community type is commonly associated with the Coulee Slopes, Eroding Slopes and the various forested community types, of which the Native Grassland community type is often adjacent on the uplands above the slope breaks, or the Native Grass/shrub Complex community type, with which it may intergrade on upland areas. The Shrub Draw community type often dissect the Native Grassland type as well.

The following is a list of plants found within this community type based upon recorded data from 35 sites.

Species

Shrub: $\quad$ Atriplex nuttallii

Potentilla fruticosa

Rosa acicularis

Rosa arkansana

Rosa woodsii

Symphoricarpos occidentalis

Forb: $\quad$ Achillea millefolium

Agoseris glauca

Allium cernuum

Allium textile

Anemone cylindrica

Anemone multifida

Anemone patens

Antennaria aprica

Antennaria parvifolia

Arabis divaricarpa

Arabis hirsuta

Arenaria congesta

Artemisia campestris

Artemisia dracunculus

Artemisia frigida

Artemisia ludoviciana

Aster ciliolatus

Aster ericoides

Aster falcatus

\section{Common name}

Nuttall's atriplex

shrubby cinquefoil

prickly rose

prairie rose

common wild rose

buckbrush

common yarrow

yellow false dandelion

nodding onion

prairie onion

long-fruited anemone

cut-leaved anemone

prairie crocus

low everlasting

small-leaved everlasting

purple rock cress

hairy rock cress

rocky-ground sandwort

plains wormwood

dragonwort

pasture sagewort

prairie sagewort

Lindley's aster

tufted white prairie aster

creeping white prairie aster 
Aster laevis

Astragalus dasyglottis

Astragalus striatus

Besseya wyomingensis

Campanula rotundifolia

Cerastium arvense

Cirsium undulatum

Chenopodium album

Comandra umbellata

Crepis runcinata

Descurainia pinnata

Dodecatheon conjugens

Draba nemorosa

Erigeron caespitosus

Erigeron glabellus

Fragaria virginiana

Gaillardia aristata

Galium boreale

Gentianella amarella

Geum triflorum

Grindelia squarrosa

Gutierrezia sarothrae

Haplopappus spinulosus

Hedysarum alpinum

Hedysarum boreale

Heterotheca villosa

Heuchera richardsonii

Hieracium umbellatum

Hymenoxys richardsonii

Lathyrus ochroleucus

Linum lewisii

Lupinus argenteus

Lygodesmia juncea

Orthocarpus luteus

Oxytropis sericea

Oxytropis splendens

Penstemon procerus

Phlox hoodii

Potentilla argentea

Potentilla concinna

Potentilla gracilis

Potentilla hippiana smooth aster

Drummond's milk vetch

ascending purple milk vetch

kittentails

harebell

field mouse-ear chickweed

wavy-leaved thistle

lamb's-quarters

bastard toadflax

scapose hawk's-beard

green tansy mustard

mountain shooting star

annual whitlow-grass

tufted fleabane

smooth fleabane

wild strawberry

gaillardia

northern bedstraw

felwort

three-flowered avens

gumweed

broomweed

spiny ironplant

alpine hedysarum

northern hedysarum

golden aster

Richardson's alumroot

narrow-leaved hawkweed

Colorado rubber plant

cream-colored vetchling

wild blue flax

silvery perennial lupine

skeletonweed

owl-clover

early yellow locoweed

showy locoweed

slender blue beardtongue

moss phlox

silvery cinquefoil

early cinquefoil

graceful cinquefoil

woolly cinquefoil 
Potentilla pensylvanica

Ranunculus cardiophyllus

Ratibida columnifera

Rhinanthus minor

Selaginella densa

Senecio canus

Silene drummondii

Sisyrinchium montanum

Solidago missouriensis

Solidago rigida

Sphaeralcea coccinea

Stellaria longifolia

Taraxacum officinale

Thermopsis rhombifolia

Tragopogon dubius

Vicia americana

Viola nuttallii

Zigadenus elegans

Zigadenus venenosus

Zizia aptera

Graminoid: Agropyron albicans

Agropyron dasystachyum

Agropyron smithii

Agropyron trachycaulum

Agrostis scabra

Bouteloua gracilis

Bromus inermis

Carex aenea

Carex filifolia

Carex obtusata

Carex pensylvanica

Carex praegracilis

Carex rostrata

Carex stenophylla

Danthonia californica

Festuca saximontana

Festuca scabrella

Helictotrichon hookeri

Juncus balticus

Koeleria macrantha

Muhlenbergia richardsonis prairie cinquefoil

heart-leaved buttercup

prairie coneflower

yellow rattle

prairie selaginella

prairie groundsel

Drummond's cockle

common blue-eyed grass

low goldenrod

stiff goldenrod

scarlet mallow

long-leaved chickweed

common dandelion

golden bean

common goat's-beard

wild vetch

yellow prairie violet

white camas

death camas

heart-leaved Alexanders

awned northern wheat grass

northern wheat grass

western wheat grass

slender wheat grass

rough hair grass

blue grama

awnless brome

silvery-flowered sedge

thread-leaved sedge

blunt sedge

sun-loving sedge

graceful sedge

beaked sedge

low sedge

California oat grass

Rocky Mountain fescue

rough fescue

Hooker's oat grass

wire rush

June grass

mat muhly 


$\begin{array}{ll}\text { Phleum pratense } & \text { timothy } \\ \text { Poa arida } & \text { plains bluegrass } \\ \text { Poa compressa } & \text { Canada bluegrass } \\ \text { Poa interior } & \text { inland bluegrass } \\ \text { Poa juncifolia } & \text { alkali bluegrass } \\ \text { Poa pratensis } & \text { Kentucky bluegrass } \\ \text { Poa sandbergii } & \text { Sandberg bluegrass } \\ \text { Stipa columbiana } & \text { Columbia needle grass } \\ \text { Stipa comata } & \text { needle-and-thread } \\ \text { Stipa curtiseta } & \text { western porcupine grass } \\ \text { Stipa viridula } & \text { green needle grass }\end{array}$

A plant species of note is death camas (Zigadenus venenosus), which contains alkaloids concentrated particularly in the bulbs of the plant and which are toxic to livestock, though cattle are less affected than sheep (U.S.D.A. 1937, Johnston et al. 1975). As well, various species of locoweed (Oxytropis sericea and O. splendens), plants which are a narcotic to cattle and causes locoism, occur at some sites within the unit (Johnston et al. 1975).

Plant species of value to wildlife include common wild rose and buckbrush, both of which are good browse species for deer, and small mammals, songbirds and grouse feed on rose hips (Stubbendieck et al. 1986, U.S.D.A. 1937). Nuttall's atriplex (Atriplex nuttallii) provides valuable winter browse for all classes of livestock, and scarlet mallow (Sphaeralcea coccinea) offers excellent forage value for deer (Stubbendieck et al. 1986). Rough fescue is a grass with high forage value for ungulates such as elk and mule deer, and June grass and northern wheat grass, along with rough fescue, provide valuable fall and winter forage (Stubbendieck et al. 1986, Looman 1983, U.S.D.A. 1937).

\subsection{Native Grass/Shrub Complex}

The Native Grass/Shrub Complex community type is, as its name suggests, a complex of native grassland and shrub communities, with shrubs comprising a significant portion of the unit, occupying 40 percent or more of the area. The community type is largely located on 
upland areas in the western half of the study area, often on inclined or rolling to ridged terrain. The Native Grass/Shrub Complex type is of relatively minor occurrence in the study area. Soils are predominantly Chernozems, ranging from Dark Brown at lower elevations or drier sites to Black at higher elevations or moister sites (Greenlee 1981, Leskiw 1990, Wyatt et al. 1941). Soil textures are commonly sandy loam. Nutrient regime is generally mesotrophic.

Plant species composition of the Native Grass/Shrub Complex community type is subject to the same controlling factors as described for the Native Grassland community type in Section 4.1 , specifically, physiography, elevation and the associated effects on microclimate and moisture regime, and grazing. However, the unit is generally no drier than submesic. As such, grassland portions of the unit tend to support grassland communities transitional to the Festuca scabrella Association as described by Coupland and Brayshaw (1953) and others. Grassland portions on relatively drier sites tend to be dominated by western porcupine grass with secondary amounts of rough fescue. Rough fescue becomes prevalent on moister sites. Shrub communities commonly consist of buckbrush (Symphoricarpos occidentalis), common wild rose (Rosa woodsii) and shrubby cinquefoil (Potentilla fruticosa) in various combinations and patterns. Associated forbs are numerous, and often include perennial silvery lupine, stiff goldenrod (Solidago rigida) and common yarrow (Achillea millefolium). Non-native species such as Kentucky bluegrass (Poa pratensis), timothy (Phleum pratense), awnless brome (Bromus inermis), common dandelion (Taraxacum officinale) and tall buttercup (Ranunculus acris), a noxious weed, can be found on sites disturbed by past cultivation or prolonged heavy grazing.

The following is a list of plants found within this community type based upon recorded data from two sites. 
$\underline{\text { Species }}$

Shrub: $\quad$ Potentilla fruticosa

Rosa arkansana

Rosa woodsii

Symphoricarpos occidentalis

Forb: $\quad$ Achillea millefolium

Agoseris glauca

Anemone cylindrica

Anemone patens

Antennaria parvifolia

Artemisia dracunculus

Artemisia frigida

Artemisia ludoviciana

Aster falcatus

Aster laevis

Astragalus dasyglottis

Campanula rotundifolia

Cerastium arvense

Fragaria virginiana

Galium boreale

Gentianella amarella

Geranium viscosissimum

Geum triflorum

Hedysarum boreale

Lathyrus ochroleucus

Lupinus argenteus

Penstemon procerus

Potentilla gracilis

Ranunculus acris

Sisyrinchium montanum

Solidago rigida

Solidago spathulata

Taraxacum officinale

Thalictrum venulosum

Thermopsis rhombifolia

Tragopogon dubius

Vicia americana

Zizia aptera

Graminoid: Agropyron dasystachyum Agropyron trachycaulum
Common name

shrubby cinquefoil

prairie rose

common wild rose

buckbrush

common yarrow

yellow false dandelion

long-fruited anemone

prairie crocus

small-leaved everlasting

dragonwort

pasture sagewort

prairie sagewort

creeping white prairie aster

smooth aster

Drummond's milk vetch

harebell

field mouse-ear chickweed

wild strawberry

northern bedstraw

felwort

sticky purple geranium

three-flowered avens

northern hedysarum

cream-colored vetchling

silvery perennial lupine

slender blue beardtongue

graceful cinquefoil

tall buttercup

common blue-eyed grass

stiff goldenrod

mountain goldenrod

common dandelion

veiny meadow rue

golden bean

common goat's-beard

wild vetch

heart-leaved Alexanders

northern wheat grass

slender wheat grass 


Agrostis scabra
Bromus inermis
Carex obtusata
Carex pensylvanica
Festuca scabrella
Helictotrichon hookeri
Koeleria macrantha
Muhlenbergia richardsonis
Phleum pratense
Poa interior
Poa pratensis
Stipa curtiseta

rough hair grass awnless brome blunt sedge sun-loving sedge rough fescue Hooker's oat grass June grass mat muhly timothy inland bluegrass Kentucky bluegrass western porcupine grass

The extensive shrub cover found in this unit gives the unit high wildlife value in the provision of food and cover for many types of wildlife species, including ungulates, small mammals and birds.

\subsection{Coulee Slopes}

The Coulee Slopes community type is essentially a grassland community type with inclusions of minor shrub draws or with shrubs comprising less than 40 percent of the unit. The community type is situated on the slopes leading to the various major coulees in the study area, including Medicine Lodge Coulee, Gros Ventre Creek and Manyberries Creek valleys. Macrotopography is inclined, with slopes up to very strong (31 to 45 percent slope). Microtopography is highly variable due to past, extensive slumping which has modified the coulee slopes since glaciation, though active slumping is currently infrequent within the unit. Plant species composition is highly variable as a result. Slope and aspect influences are of major importance in controlling the plant species composition at any given site within the community type. Ecological moisture regime ranges from subxeric to subhygric, depending on aspect, slope position, or both. South-facing slopes are often the driest, being subxeric due to their greater exposure to sunlight and, on southwest-facing slopes, exposure to prevailing winds at the hottest portion of the day. However, lower slopes and valley bottoms 
of any aspect are often submesic to subhygric due to the influence of subsurface water flow or of non-saline and saline springs. Nutrient regime in the unit is generally mesotrophic, becoming permesotrophic on some lower slope positions and in valley bottoms. The unit is generally well drained.

Drier sites such as found on southwest-facing sites or south-facing upper slopes support plant species more typical of western porcupine grass-dominated communities as described in Section 4.1. Moister submesic to mesic sites common on north-facing slopes or on other middle and lower slopes support plants species more typical of rough fescue-dominated communities also described earlier. Often, the plant community at a given site is transitional between the two types of communities. Subhygric sites, as found at seepage sites at the base of lower slopes, support communities dominated by species such as rough hair grass (Agrostis scabra), wire rush (Juncus balticus) and common yarrow. The community type tends to support numerous forb species. Shrubs which occur in minor shrub draws and minor depressions often include saskatoon (Amelanchier alnifolia), choke cherry (Prunus virginiana), round-leaved hawthorn (Crataegus rotundifolia), common wild rose, buckbrush and northern gooseberry (Ribes oxyacanthoides). Shrubs on drier, south-facing slopes can include silver sagebrush (Artemisia cana), rabbitbrush (Chrysothamnus nauseosus) and creeping juniper (Juniperus horizontalis). Occasional trees, such as white spruce (Picea glauca) can be found where the Coulee Slopes community type intergrades with forested community types.

The Coulee Slopes community type is often associated with the Native Grassland community type, usually present on upland areas above the Coulee Slopes type; the Eroding Slopes community type which occurs on steeper, actively eroding coulee slopes; the various forested community types which can occur at higher elevations and northern aspects on similar terrain to the Coulee Slopes type; and the Alluvial Fans/Floodplains community type often occurring on valley bottoms below the Coulee Slopes type. 
The following are lists of plants found within this community type based upon recorded data from 25 sites.

\section{Species}

Tree: $\quad$ Picea glauca

Shrub: $\quad$ Artemisia cana

Chrysothamnus nauseosus

Crataegus rotundifolia

Juniperus horizontalis

Potentilla fruticosa

Prunus virginiana

Ribes oxyacanthoides

Rosa arkansana

Rosa woodsii

Symphoricarpos occidentalis

Forb: $\quad$ Achillea millefolium

Agoseris glauca

Androsace septentrionalis

Anemone multifida

Anemone patens

Antennaria parvifolia

Arabis hirsuta

Arabis holboellii

Arenaria congesta

Artemisia campestris

Artemisia frigida

Artemisia ludoviciana

Aster ascendens

Aster ericoides

Aster falcatus

Aster laevis

Astragalus bisulcatus

Astragalus dasyglottis

Astragalus pectinatus

Astragalus striatus

Campanula rotundifolia

Cerastium arvense

Chenopodium album

Cirsium flodmanii

\section{Common name}

white spruce

silver sagebrush

rabbitbrush

round-leaved hawthorn

creeping juniper

shrubby cinquefoil

choke cherry

northern gooseberry

prairie rose

common wild rose

buckbrush

common yarrow

yellow false dandelion

northern fairy candelabra

cut-leaved anemone

prairie crocus

small-leaved everlasting

hairy rock cress

reflexed rock cress

rocky-ground sandwort

plains wormwood

pasture sagewort

prairie sagewort

western aster

tufted white prairie aster

creeping white prairie aster

smooth aster

two-grooved milk vetch

Drummond's milk vetch

narrow-leaved milk vetch

ascending purple milk vetch

harebell

field mouse-ear chickweed

lamb's-quarters

Flodman's thistle 
Cirsium vulgare

Collomia linearis

Comandra umbellata

Crepis runcinata

Dodecatheon pulchellum

Epilobium angustifolium

Erigeron caespitosus

Eriogonum flavum

Erysimum inconspicuum

Fragaria virginiana

Gaillardia aristata

Galium boreale

Gentianella amarella

Geranium viscosissimum

Geum triflorum

Glycyrrhiza lepidota

Grindelia squarrosa

Gutierrezia sarothrae

Haplopappus spinulosus

Hedeoma hispidum

Heracleum lanatum

Heterotheca villosa

Heuchera richardsonii

Lathyrus ochroleucus

Liatris punctata

Lithospermum ruderale

Lupinus argenteus

Medicago sativa

Orthocarpus luteus

Oxytropis sericea

Penstemon procerus

Phlox hoodii

Polygonum arenastrum

Polygonum douglasii

Potentilla argentea

Potentilla concinna

Potentilla gracilis

Potentilla hippiana

Potentilla pensylvanica

Ratibida columnifera

Rhinanthus minor

Selaginella densa bull thistle

narrow-leaved collomia

bastard toadflax

scapose hawk's-beard

saline shooting star

common fireweed

tufted fleabane

yellow umbrella-plant

small-flowered rocket

wild strawberry

gaillardia

northern bedstraw

felwort

sticky purple geranium

three-flowered avens

wild licorice

gumweed

broomweed

spiny ironplant

pennyroyal

cow parsnip

golden aster

Richardson's alumroot

cream-colored vetchling

dotted blazingstar

woolly gromwell

silvery perennial lupine

alfalfa

owl-clover

early yellow locoweed

slender blue beardtongue

moss phlox

common knotweed

Douglas knotweed

silvery cinquefoil

early cinquefoil

graceful cinquefoil

woolly cinquefoil

prairie cinquefoil

prairie coneflower

yellow rattle

prairie selaginella 
Senecio canus

Silene drummondii

Sisyrinchium montanum

Smilacina stellata

Solidago gigantea

Solidago missouriensis

Sphaeralcea coccinea

Stellaria longifolia

Taraxacum officinale

Thalictrum venulosum

Thermopsis rhombifolia

Tragopogon dubius

Vicia americana

Viola adunca

Graminoid: Agropyron albicans

Agropyron dasystachyum

Agropyron pectiniforme

Agropyron smithii

Agropyron trachycaulum

Agrostis scabra

Bromus carinatus

Bromus ciliatus

Bromus inermis

Bouteloua gracilis

Calamagrostis montanensis

Calamovilfa longifolia

Carex aenea

Carex filifolia

Carex obtusata

Carex pensylvanica

Carex praegracilis

Carex stenophylla

Danthonia californica

Deschampsia cespitosa

Festuca scabrella

Helictotrichon hookeri

Hordeum jubatum

Juncus balticus

Juncus confusus

Koeleria macrantha

Muhlenbergia richardsonis prairie groundsel

Drummond's cockle

common blue-eyed grass

star-flowered Solomon's-seal

late goldenrod

low goldenrod

scarlet mallow

long-leaved chickweed

common dandelion

veiny meadow rue

golden bean

common goat's-beard

wild vetch

early blue violet

awned northern wheat grass

northern wheat grass

crested wheat grass

western wheat grass

slender wheat grass

rough hair grass

keeled brome

fringed brome

awnless brome

blue grama

plains reed grass

sand grass

silvery-flowered sedge

thread-leaved sedge

blunt sedge

sun-loving sedge

graceful sedge

low sedge

California oat grass

tufted hair grass

rough fescue

Hooker's oat grass

foxtail barley

wire rush

few-flowered rush

June grass

mat muhly 


$\begin{array}{ll}\text { Phleum pratense } & \text { timothy } \\ \text { Poa arida } & \text { plains bluegrass } \\ \text { Poa compressa } & \text { Canada bluegrass } \\ \text { Poa interior } & \text { inland bluegrass } \\ \text { Poa juncifolia } & \text { alkali bluegrass } \\ \text { Poa sandbergii } & \text { Sandberg bluegrass } \\ \text { Poa palustris } & \text { fowl bluegrass } \\ \text { Poa pratensis } & \text { Kentucky bluegrass } \\ \text { Stipa columbiana } & \text { Columbia needle grass } \\ \text { Stipa comata } & \text { needle-and-thread } \\ \text { Stipa curtiseta } & \text { western porcupine } \\ \text { Stipa viridula } & \text { green needle grass } \\ \text { Spartina gracilis } & \text { alkali cord grass }\end{array}$

Plant species found on submesic to mesic north-facing slopes include:

Species

Tree: $\quad$ Picea glauca

Shrub: $\quad$ Amelanchier alnifolia

Crataegus douglasii

Crataegus rotundifolia

Elaeagnus commutata

Prunus virginiana

Ribes oxyacanthoides

Rosa woodsii

Rubus idaeus

Shepherdia canadensis

Symphoricarpos occidentalis

Forb: $\quad$ Achillea millefolium

Artemisia ludoviciana

Aster hesperius

Aster laevis

Cerastium arvense

Cirsium arvense

Disporum trachycarpum

Fragaria virginiana

Galium boreale

Geum aleppicum

Glycyrrhiza lepidota

Lithospermum ruderale
Common name

white spruce

saskatoon

Douglas hawthorn

round-leaved hawthorn

silverberry

choke cherry

northern gooseberry

common wild rose

wild red raspberry

Canada buffaloberry

buckbrush

common yarrow

prairie sagewort

western willow aster

smooth aster

field mouse-ear chickweed

Canada thistle

fairybells

wild strawberry

northern bedstraw

yellow avens

wild licorice

woolly gromwell 
Osmorhiza depauperata

Perideridia gairdneri

Ranunculus macounii

Rumex triangulivalvis

Smilacina stellata

Solidago gigantea

Stachys palustris

Taraxacum officinale

Thlaspi arvense

Urtica dioica

Vicia americana

Viola canadensis

Graminoid: Agropyron trachycaulum

Bromus carinatus

Bromus ciliatus

Bromus inermis

Calamagrostis stricta

Carex aenea

Carex praegracilis

Carex rostrata

Carex sprengelii

Cinna latifolia

Deschampsia cespitosa

Glyceria striata

Phleum pratense

Poa interior

Poa palustris

Poa pratensis

Stipa viridula spreading sweet cicely

squawroot

Macoun's buttercup

narrow-leaved dock

star-flowered Solomon's-seal

late goldenrod

marsh hedge-nettle

common dandelion

stinkweed

common nettle

wild vetch

western Canada violet

slender wheat grass

keeled brome

fringed brome

awnless brome

narrow reed grass

silvery-flowered sedge

graceful sedge

beaked sedge

Sprengel's sedge

drooping wood-reed

tufted hair grass

fowl manna grass

timothy

inland bluegrass

fowl bluegrass

Kentucky bluegrass

green needle grass

Pennyroyal (Hedeoma hispidum), rare in Alberta, was found in the Coulee Slopes community type on an east-facing, well-drained, submesic lower slope of Medicine Lodge Coulee during 1995 field investigations. 
Rough fescue is a grass with high forage value for ungulates such as elk and mule deer, and June grass and northern wheat grass, along with rough fescue, provide valuable fall and winter forage (Stubbendieck et al. 1986, Looman 1983).

Other plant species of note include scarlet globemallow, which provides excellent forage value for deer, and blue grama and western wheatgrass which provide good to excellent forage value for grazing wildlife (Stubbendieck et al. 1986).

Two-grooved milk vetch (Astragalus bisulcatus) is considered an indicator of the presence of selenium in soils, and Cretaceous shales which contain selenium occur around the periphery of the Cypress Hills. This is an important management concern to livestock producers as the occurrence of selenium and its accumulation in some plant species, including Astragalus bisulcatus, can result in livestock losses (Johnston et al. 1975, Looman 1983).

\subsection{Eroding Slopes}

The Eroding Slopes community type occurs on slopes of the major coulees and tributary drainages within the study area, often in association with the Coulee Slopes community type. Active slumping and mass wasting is common in the Eroding Slopes community type, and slopes are usually extreme (45 to 70 percent slope) or greater. Microtopography is complex and highly variable. Exposed mineral soil, and occasionally bedrock, is common. The effects of salinity can be pronounced within the southern portion of the study area underlain by the marine Bearpaw Formation, and Solonetzic soils can be expected here, with slopes less steep than much of the unit, often less than very strong (31 to 45 percent slope). The effects of water erosion are particularly manifested in these areas, with sheet and rill erosion usually transporting material to the Alluvial Fans/Floodplain community type immediately downslope. 
Soil textures are variable ranging from sandy loam or sandy clay loam to silty clay. The unit is predominantly well drained. Soils are expected to include Orthic Regosols and non-soils and weakly developed Dark Brown Chernozems on more gentle slopes, with Solonetzic soils likely in southern portions of the study area as described above. Ecological moisture regime is variable, ranging from very xeric to subxeric on southern aspects, to submesic and mesic on northern aspects. Lower slopes and valley bottoms of any aspect are often mesic to subhygric from the influence of subsurface water flow and of non-saline and saline springs. Nutrient regime is variable, commonly mesotrophic but can be submesotrophic on very xeric sites. The Eroding Slopes community type excludes forested vegetation types which can occur on similar terrain at higher elevations.

Vegetation within the Eroded Slopes community type is highly variable as a result of the factors described above. Vegetation on very xeric south-facing slopes consists of species such as creeping juniper, silver sagebrush, needle-and-thread (Stipa comata), thread-leaved sedge (Carex filifolia) and sand grass (Calamovilfa longifolia). Conversely, submesic northeast-facing slopes can support communities consisting of rough fescue, buckbrush, common wild rose, smooth aster (Aster laevis) and northern bedstraw (Galium boreale).

The following are lists of plants found within this community type based upon recorded data from 22 sites.

\section{Species}

Shrub:
Amelanchier alnifolia

Artemisia cana

Crataegus rotundifolia

Elaeagnus commutata

Juniperus communis

Juniperus horizontalis

Potentilla fruticosa

Prunus virginiana

Ribes oxyacanthoides

\section{Common name}

saskatoon

silver sagebrush

round-leaved hawthorn

silverberry

ground juniper

creeping juniper

shrubby cinquefoil

choke cherry

northern gooseberry 
Rosa arkansana

Rosa woodsii

Shepherdia canadensis

Spiraea betulifolia

Symphoricarpos occidentalis

Forb: $\quad$ Achillea millefolium

Allium cernuum

Androsace septentrionalis

Anemone cylindrica

Anemone multifida

Anemone patens

Antennaria parvifolia

Arabis hirsuta

Arenaria congesta

Artemisia campestris

Artemisia frigida

Artemisia ludoviciana

Aster ericoides

Aster falcatus

Aster hesperius

Aster laevis

Astragalus dasyglottis

Astragalus drummondii

Astragalus gilviflorus

Astragalus spatulatus

Astragalus striatus

Astragalus tenellus

Astragalus vexilliflexus

Besseya wyomingensis

Campanula rotundifolia

Cerastium arvense

Chenopodium album

Cirsium arvense

Collomia linearis

Comandra umbellata

Crepis runcinata

Descurainia pinnata

Disporum trachycarpum

Draba nemorosa

Epilobium paniculatum

Erigeron caespitosus prairie rose

common wild rose

Canada buffaloberry

white meadowsweet

buckbrush

common yarrow

nodding onion

northern fairy candelabra

long-fruited anemone

cut-leaved anemone

prairie crocus

small-leaved everlasting

hairy rock cress

rocky-ground sandwort

plains wormwood

pasture sagewort

prairie sagewort

tufted white prairie aster

creeping white prairie aster

western willow aster

smooth aster

purple milk vetch

Drummond's milk vetch

cushion milk vetch

tufted milk vetch

ascending purple milk vetch

loose-flowered milk vetch

few-flowered milk vetch

kittentails

harebell

field mouse-ear chickweed

lamb's-quarters

Canada thistle

narrow-leaved collomia

bastard toadflax

scapose hawk's-beard

green tansy mustard

fairybells

annual whitlow-grass

annual willow-herb

tufted fleabane 
Erigeron glabellus

Eriogonum flavum

Erysimum inconspicuum

Fragaria virginiana

Gaillardia aristata

Galium boreale

Gentianella amarella

Geranium viscosissimum

Geum aleppicum

Geum triflorum

Glycyrrhiza lepidota

Grindelia squarrosa

Gutierrezia sarothrae

Hackelia floribunda

Hedysarum alpinum

Hedysarum boreale

Helianthus nuttallii

Heracleum lanatum

Heterotheca villosa

Heuchera richardsonii

Hymenoxys richardsonii

Lathyrus ochroleucus

Liatris punctata

Linum lewisii

Lithospermum ruderale

Lomatium macrocarpum

Lupinus argenteus

Melilotus alba

Melilotus officinalis

Monarda fistulosa

Osmorhiza depauperata

Oxytropis sericea

Penstemon procerus

Petalostemon purpureum

Phlox hoodii

Potentilla argentea

Potentilla concinna

Potentilla hippiana

Potentilla pensylvanica

Polygonum arenastrum

Polygonum douglasii

Potentilla gracilis smooth fleabane

yellow umbrella plant

small-flowered rocket

wild strawberry

gaillardia

northern bedstraw

felwort

sticky purple geranium

yellow avens

three-flowered avens

wild licorice

gumweed

broomweed

large-flowered stickseed

alpine hedysarum

northern hedysarum

common tall sunflower

cow parsnip

golden aster

Richardson's alumroot

Colorado rubber plant

cream-colored vetchling

dotted blazingstar

wild blue flax

woolly gromwell

long-fruited wild parsley

silvery perennial lupine

white sweet-clover

yellow sweet-clover

wild bergamot

spreading sweet cicely

early yellow locoweed

slender blue beardtongue

purple prairie-clover

moss phlox

silvery cinquefoil

early cinquefoil

woolly cinquefoil

prairie cinquefoil

common knotweed

Douglas knotweed

graceful cinquefoil 
Ratibida columnifera

Salsola kali

Sedum lanceolatum

Selaginella densa

Senecio canus

Silene drummondii

Sisyrinchium montanum

Smilacina stellata

Solidago gigantea

Solidago missouriensis

Solidago rigida

Solidago spathulata

Taraxacum officinale

Thermopsis rhombifolia

Tragopogon dubius

Urtica dioica

Vicia americana

Viola adunca

Zizia aptera

Graminoid: Agropyron dasystachyum Agropyron smithii Agropyron trachycaulum Agrostis scabra Bouteloua gracilis

Calamovilfa longifolia

Carex aenea

Carex filifolia

Carex obtusata

Carex pensylvanica

Carex sprengelii

Carex stenophylla

Danthonia californica

Elymus canadensis

Festuca saximontana

Festuca scabrella

Helictotrichon hookeri

Juncus balticus

Koeleria macrantha

Muhlenbergia richardsonis

Oryzopsis hymenoides

Phleum pratense prairie coneflower

Russian thistle

lance-leaved stonecrop

prairie selaginella

prairie groundsel

Drummond's cockle

common blue-eyed grass

star-flowered Solomon's-seal

late goldenrod

low goldenrod

stiff goldenrod

mountain goldenrod

common dandelion

golden bean

common goat's-beard

common nettle

wild vetch

early blue violet

heart-leaved Alexanders

northern wheat grass

western wheat grass

slender wheat grass

rough hair grass

blue grama

sand grass

silvery-flowered sedge

thread-leaved sedge

blunt sedge

sun-loving sedge

Sprengel's sedge

low sedge

California oat grass

Canada wild rye

Rocky Mountain fescue

rough fescue

Hooker's oat grass

wire rush

June grass

mat muhly

Indian rice grass

timothy 
Poa arida

Poa compressa

Poa interior

Poa juncifolia

Poa pratensis

Poa sandbergii

Stipa columbiana

Stipa comata

Stipa curtiseta

Stipa viridula plains bluegrass

Canada bluegrass

plains bluegrass

alkali bluegrass

Kentucky bluegrass

Sandberg bluegrass

Columbia needle grass

needle-and-thread

western porcupine grass

green needle grass

North-facing lower slopes and valley bottom sites can support the following plant species characteristic of moist sites:

Species

Tree:

Picea glauca

Populus balsamifera

Populus tremuloides

Shrub: $\quad$ Amelanchier alnifolia

Cornus stolonifera

Prunus virginiana

Ribes oxyacanthoides

Rosa acicularis

Rubus idaeus

Symphoricarpos occidentalis

Forb: $\quad$ Achillea millefolium

Artemisia ludoviciana

Aster falcatus

Aster laevis

Cerastium arvense

Cirsium arvense

Cirsium flodmanii

Cirsium vulgare

Collomia linearis

Erysimum cheiranthoides

Fragaria virginiana

Galium boreale

Geranium viscosissimum

\section{Common name}

white spruce

balsam poplar

aspen

saskatoon

red-osier dogwood

choke cherry

northern gooseberry

prickly rose

wild red raspberry

buckbrush

common yarrow

prairie sagewort

creeping white prairie aster

smooth aster

field mouse-ear chickweed

Canada thistle

Flodman's thistle

bull thistle

narrow-leaved collomia

wormseed mustard

wild strawberry

northern bedstraw

sticky purple geranium 
Geum aleppicum

Hieracium umbellatum

Lactuca pulchellum

Lathyrus ochroleucus

Mentha arvensis

Monarda fistulosa

Osmorhiza depauperata

Smilacina stellata

Solidago gigantea

Solidago missouriensis

Taraxacum officinale

Thalictrum dasycarpum

Urtica dioica

Vicia americana

Viola canadensis

Graminoid: Agropyron trachycaulum

Bromus ciliatus

Bromus inermis

Calamagrostis inexpansa

Carex sprengelii

Poa compressa

Poa interior

Poa pratensis

yellow avens
narrow-leaved hawkweed
common blue lettuce
cream-colored vetchling
wild mint
wild bergamot
spreading sweet cicely
star-flowered Solomon's-seal
late goldenrod
low goldenrod
common dandelion
tall meadow rue
common nettle
wild vetch
western Canada violet
slender wheat grass
fringed brome
awnless brome
northern reed grass
Sprengel's sedge
Canada bluegrass
inland bluegrass
Kentucky bluegrass

yellow avens

narrow-leaved hawkweed

common blue lettuce

cream-colored vetchling

wild mint

wild bergamot

spreading sweet cicely

star-flowered Solomon's-seal

late goldenrod

low goldenrod

common dandelion

tall meadow rue

common nettle

wild vetch

This community type contains many species which are important as browse, forage and cover for many wildlife species, including mule deer, small mammals and birds. Silver sagebrush, chokecherry and pasture sagewort, to name but a few provide important winter feed for mule deer. Common wild rose provides good browse for mule deer, and small mammals and birds feed on rose hips (Stubbendieck 1986).

\subsection{Alluvial Fans/Floodplains}

The primary defining characteristic of the Alluvial Fans/Floodplains community type is that the unit is a deposition area for mineral material transported by water. Areas where this community type occurs include the wide floodplains present on the valley bottoms of various 
glacial meltwater channels within the study area which now contain underfit streams. Such areas include Medicine Lodge Coulee and its tributary drainages, plus Gros Ventre Creek and Manyberries Creek valleys. Alluvial fans extend into the valley bottoms as well, serving as outwash areas for mineral material eroded from adjacent upland units. Aspects are variable depending on the orientation of the valleys. The Riparian community type is usually located adjacent to and downslope from the unit.

The effects of salinity can be pronounced within Medicine Lodge Coulee and Manyberries Creek valley in the southern portion of the study area underlain by the marine Bearpaw Formation, and Solonetzic soils can be expected here. Parent materials are fluvial, with soil textures generally finer than those of upland areas, ranging from silty clay to silt loam to sandy clay loam. Depth to water table can be shallow. Drainage is variable, ranging from imperfectly to poorly drained on floodplains and some fans, to well to rapidly drained on other fan areas. Ecological moisture regimes are variable as well, from subhygric to xeric depending on soil textures and drainage. Slopes are generally level ( 0 to 0.5 percent slope) to nearly level ( 0.5 to 2.5 percent slope) on floodplains, with slopes on fans ranging up to moderate (10 to 15 percent slope).

The Alluvial Fans/Floodplains community type is essentially a grassland community type. The plant community present at a given site is largely dependent on moisture regime, salinity and disturbance by deposition, grazing or past cultivation. Non-native species such as Kentucky bluegrass, Canada bluegrass (Poa compressa), awnless brome, white sweet-clover (Melilotus alba) and yellow sweet-clover (M. officinalis) are often important components of the community due to grazing history or past cultivation. Foxtail barley (Hordeum jubatum), alkali bluegrass (Poa juncifolia), salt grass (Distichlis stricta) and gumweed (Grindelia squarrosa), along with Kentucky bluegrass, are often found on poorly drained saline sites. On relatively drier areas, the plant community becomes similar to those portions of the Native Grassland community type where western wheat grass is prevalent. Mat muhly 
(Muhlenbergia richardsonis), slender wheat grass (Agropyron trachycaulum), June grass, common yarrow, pasture sagewort (Artemisia frigida), golden aster (Heterotheca villosa) and small-leaved everlasting (Antennaria parvifolia) are some associated species at these sites.

Mousetail (Myosurus aristatus), rare in Alberta, was found within this community type on a subhygric, saline, sandy clay alluvial fan in the vicinity of Bullshead Creek during 1995 field investigations.

The following is a list of plants found within this community type based upon recorded data from 10 sites.

$\underline{\text { Species }}$

Shrub:

Forb:
Artemisia cana

Atriplex nuttallii

Chrysothamnus nauseosus

Rosa woodsii

Sarcobatus vermiculatus

Symphoricarpos occidentalis

Achillea millefolium

Antennaria parvifolia

Arenaria congesta

Artemisia frigida

Artemisia ludoviciana

Aster ascendens

Aster ericoides

Aster falcatus

Aster laevis

Descurainia pinnata

Erigeron compositus

Erigeron glabellus

Eurotia lanata

Gentianella amarella

Geum triflorum

Grindelia squarrosa

Gutierrezia sarothrae

\section{Common name}

silver sagebrush

Nuttall's atriplex

rabbitbrush

common wild rose

greasewood

buckbrush

common yarrow

small-leaved everlasting

rocky-ground sandwort

pasture sagewort

prairie sagewort

western aster

tufted white prairie aster

creeping white prairie aster

smooth aster

green tansy mustard

compound-leaved fleabane

smooth fleabane

winter-fat

felwort

three-flowered avens

gumweed

broomweed 
Haplopappus lanceolatus

Helianthus nuttallii

Heterotheca villosa

Hymenoxys richardsonii

Iva axillaris

Lepidium densiflorum

Linum lewisii

Machaeranthera canescens

Melilotus alba

Melilotus officinalis

Myosurus aristatus

Opuntia fragilis

Orthocarpus luteus

Phlox hoodii

Plantago eriopoda

Polygonum ramosissimum

Potentilla hippiana

Potentilla pensylvanica

Ratibida columnifera

Sedum lanceolatum

Selaginella densa

Solidago missouriensis

Solidago rigida

Sphaeralcea coccinea

Suaeda calceoliformis

Taraxacum officinale

Vicia americana

Graminoid: Agrohordeum macounii Agropyron albicans Agropyron dasystachyum Agropyron smithii Agropyron trachycaulum Agropyron pectiniforme Agrostis scabra Bromus inermis

Carex obtusata

Carex pensylvanica

Carex praegracilis

Carex stenophylla

Deschampsia cespitosa

Distichlis stricta lance-leaved ironplant common tall sunflower

golden aster

Colorado rubber-plant povertyweed

common pepper-grass

wild blue flax

hoary aster

white sweet-clover

yellow sweet-clover

mousetail

brittle prickly-pear

owl-clover

moss phlox

saline plantain

bushy knotweed

woolly cinquefoil

prairie cinquefoil

prairie coneflower

lance-leaved stonecrop

prairie selaginella

low goldenrod

stiff goldenrod

scarlet mallow

western sea-blite

common dandelion

wild vetch

Macoun's wild rye

awned northern wheat grass

northern wheat grass

western wheat grass

slender wheat grass

crested wheat grass

rough hair grass

awnless brome

blunt sedge

sun-loving sedge

graceful sedge

low sedge

tufted hair grass

salt grass 


Helictotrichon hookeri
Hordeum jubatum
Juncus balticus
Juncus confusus
Koeleria macrantha
Muhlenbergia cuspidata
Muhlenbergia richardsonis
Phleum pratense
Poa arida
Poa compressa
Poa interior
Poa juncifolia
Poa palustris
Poa pratensis
Puccinellia nuttalliana
Scirpus pungens
Spartina gracilis
Stipa comata
Stipa curtiseta
Stipa viridula

Hooker's oat grass
foxtail barley
wire rush
few-flowered rush
June grass
plains muhly
mat muhly
timothy
plains bluegrass
Canada bluegrass
inland bluegrass
alkali bluegrass
fowl bluegrass
Kentucky bluegrass
Nuttall's salt-meadow grass
three-square rush
alkali cord grass
needle-and-thread
western porcupine grass
green needle grass

The unit can be valuable for wildlife, particularly due to the sheltered valley bottom locations of the unit, to the availability of forage and to the presence of shrub species valuable as sources of food and cover.

\subsection{Hummocky Grassland}

The Hummocky Grassland community type is predominantly located in the northern portion of the study area northwest and north of the Cypress Hills Plateau. This grassland community type occurs on hummocky disintegration moraine, creating a complex "knob-and-kettle" topography of relatively low, circular or oval mounds with shallow central depressions (Westgate 1968). Slopes are complex, with areas of strong relief having complex slopes ranging from strong (16 to 30 percent slope) to very strong (31 to 45 percent). Areas with more subdued relief have complex slopes from gentle ( 6 to 9 percent) to moderate (10 to 15 percent slope). Soil textures are commonly sandy loam. Soils are expected to be 
Chernozemic, commonly Dark Brown with some occurrences of Black, on knolls, intergrading to imperfectly drained Chernozems to Gleysols depending on moisture regimes.

The Hummocky Grassland community type is quite similar to the Native Grassland community type, but is differentiated by the numerous moist, closed depressions characteristic of "knob-and-kettle" topography, creating a pattern of subxeric hill crests and submesic lower slopes supporting vegetation typical of the Native Grassland community type and subject to the same controlling factors for that type as described in Section 4.1, alternating with vegetation typical of mesic or moister ecological moisture regimes. Shrub thickets frequently consisting of buckbrush and common wild rose are common in these depressions, as are many ephemeral wetlands and occasional permanent wetlands supporting the Ephemeral Wetland and Permanent Wetland community types.

The following is a list of plants found within this community type based upon recorded data from seven sites.

\section{Species}

Shrub: $\quad$ Juniperus horizontalis Rosa arkansana

Rosa woodsii

Symphoricarpos occidentalis

Forb: $\quad$ Achillea millefolium

Androsace septentrionalis

Anemone patens

Antennaria parvifolia

Arenaria congesta

Artemisia campestris

Artemisia dracunculus

Artemisia frigida

Artemisia ludoviciana

Aster ericoides

Aster falcatus

Astragalus dasyglottis

\section{Common name}

creeping juniper

prairie rose

common wild rose

buckbrush

common yarrow

northern fairy candelabra

prairie crocus

small-leaved everlasting

rocky-ground sandwort

plains wormwood

dragonwort

pasture sagewort

prairie sagewort

tufted white prairie aster

creeping white prairie aster

Drummond's milk vetch 
Astragalus striatus

Cerastium arvense

Comandra umbellata

Erigeron caespitosus

Gaillardia aristata

Galium boreale

Geum triflorum

Grindelia squarrosa

Gutierrezia sarothrae

Heterotheca villosa

Liatris punctata

Orthocarpus luteus

Oxytropis sericea

Phlox hoodii

Potentilla concinna

Potentilla hippiana

Potentilla pensylvanica

Ratibida columnifera

Selaginella densa

Senecio canus

Silene drummondii

Solidago missouriensis

Solidago mollis

Taraxacum officinale

Thermopsis rhombifolia

Tragopogon dubius

Vicia americana

Graminoid: Agropyron dasystachyum

Agropyron pectiniforme

Agropyron trachycaulum

Agrostis scabra

Bouteloua gracilis

Carex filifolia

Carex obtusata

Carex stenophylla

Festuca scabrella

Helictotrichon hookeri

Koeleria macrantha

Poa arida

Poa pratensis

Poa sandbergii ascending purple milk vetch

field mouse-ear chickweed

bastard toadflax

tufted fleabane

gaillardia

northern bedstraw

three-flowered avens

gumweed

broomweed

golden aster

dotted blazingstar

owl-clover

early yellow locoweed

moss phlox

early cinquefoil

woolly cinquefoil

prairie cinquefoil

prairie coneflower

prairie selaginella

prairie groundsel

Drummond's cockle

low goldenrod

velvety goldenrod

common dandelion

golden bean

common goat's-beard

wild vetch

northern wheat grass

crested wheat grass

slender wheat grass

rough hair grass

blue grama

thread-leaved sedge

blunt sedge

low sedge

rough fescue

Hooker's oat grass

June grass

plains bluegrass

Kentucky bluegrass

Sandberg bluegrass 
Stipa comata

Stipa curtiseta needle-and-thread

western porcupine grass

Early yellow locoweed (Oxytropis sericea), a plant which is a narcotic to cattle and causes locoism (Johnston et al. 1975), occurs at some sites within the unit.

The Hummocky Grassland community type is expected to be of significant value to deer species in the study area, due to the hummocky topography of the unit providing shelter, and due to plant species present in this type providing valuable forage and browse.

\subsection{Wetlands}

Numerous ephemeral and permanent wetlands occur in the Eagle Butte Sensitive Area. Wetlands in the study area range in size from small potholes to relatively large water bodies created as a result of irrigation projects.

\subsubsection{Permanent Wetland}

The Permanent Wetland community type encompasses plant communities found in association with wetlands in the study area which generally contain standing water yearround, year to year. Many small permanent wetlands are located along the flanks of the Cypress Hills and are fed by springs which discharge along these slopes, often at the base of slump scars and at upper reaches of gullies (Leskiw 1990). Ponds are occasional within the backslopes of some slump blocks. As well, the damming of some drainages, largely for irrigation purposes, has created permanent reservoirs of which Michel Reservoir is the largest. In addition, the western tip of Elkwater Lake extends into the study area.

Plant communities within the Permanent Wetland community type are highly variable depending on the site. Vegetation at some spring-fed wetlands found along the flanks of the 
Cypress Hills within the Eagle's Nest Candidate Natural Area were described by Biota Consultants Ltd. and Komex International (1992), with willow (Salix spp.) occurring along wetland margins and cattail (Typha latifolia var. latifolia), common great bulrush (Scirpus validus), wire rush and various species of sedge found as emergent vegetation. Stagnant beaver ponds can be completely covered with common duckweed (Lemna minor).

The following is a list of plants found within this community type based upon recorded data from one site located adjacent the Michel Reservoir.

Species

Forb: $\quad$ Aster falcatus

Coreopsis tinctoria

Epilobium ciliatum

Grindelia squarrosa

Mentha arvensis

Plantago major

Polygonum monspeliense

Potentilla anserina

Potentilla norvegica

Rumex triangulivalvis

Sonchus arvensis

Graminoid: Beckmannia syzigachne

Deschampsia cespitosa

Eleocharis palustris

Hordeum jubatum

Juncus balticus

Phalaris arundinacea

Phleum pratense

Poa compressa

Poa palustris

Poa pratensis

\section{Common name}

creeping white prairie aster

common tickseed

northern willowherb

gumweed

wild mint

common plantain

knotweed

silverweed

rough cinquefoil

narrow-leaved dock

perennial sow-thistle

slough grass

tufted hair grass

creeping spike-rush

foxtail barley

wire rush

reed canary grass

timothy

Canada bluegrass

fowl bluegrass

Kentucky bluegrass

The Permanent Wetland community type provides important habitat for many wildlife species such as waterfowl, shorebirds and herptiles. Particularly notable are the populations of 
northern leopard frogs supported by permanent wetlands in the study area. The significance of wetlands within the study area is discussed in detail in Section 6.5.5 and Section 9.0 of this report.

\subsubsection{Ephemeral Wetland}

The Ephemeral Wetland community type has been mapped throughout the study area but are most common in association with the Hummocky Grassland community type in the northern portion of the study area, with the Ephemeral Wetland community type found in many of the numerous depressions within the hummocky disintegration moraine which occurs in this part of the study area. These wetlands are temporary in nature, poorly drained, and have a seasonally variable moisture regime, ranging from hydric in the spring to submesic and subxeric in the late summer and fall. These level depressions in the landscape are rather circular in pattern and are areas of snow accumulation during the winter period. Dugouts for livestock watering have been constructed at many locations within this community type. Nutrient status is permesotrophic, slightly more nutrient-rich than the surrounding grassland community types. Soils are expected to be predominantly Gleysolic, formed in morainal or lacustrine parent materials. Soil textures are generally heavy clays. Slopes are level to very gentle ( 0 to 2 percent slope).

Vegetation within the Ephemeral Wetland community type is highly variable within and between each site. Plant communities in the ephemeral wetlands tend to occur more or less in concentric rings. Each ring of vegetation reflects a different ecological moisture regime with a corresponding different plant community. The percentage of exposed mineral soil is highly variable, depending on the amount and timing of water draw-down.

The graminoid vegetation prevalent in the unit provides valuable forage for grazing animals. The community type is also important in providing food and cover to upland bird species 
such as sharp-tailed grouse, with ephemeral wetlands in southeast Alberta often having high value for brood-rearing (J. Clark, pers. comm.).

The following is a list of plants found within this community type based upon recorded data from seven sites.

\section{Species}

Forb: $\quad$ Artemisia biennis

Aster hesperius

Calla palustris

Epilobium ciliatum

Hippuris vulgaris

Myriophyllum exalbescens

Polygonum amphibium

Polygonum coccineum

Potamogeton filiformis

Potamogeton gramineus

Potamogeton richardsonii

Potentilla anserina

Potentilla gracilis

Potentilla norvegica

Ranunculus gmelinii

Rumex occidentalis

Sagitarria cuneata

Stachys palustris

Taraxacum officinale

Trifolium hybridum

Utricularia vulgaris

Graminoid: Agrostis scabra

Beckmannia syzigachne

Calamagrostis stricta

Carex aquatilis

Carex atherodes

Carex lanuginosa

Carex praegracilis

Deschampsia cespitosa

Eleocharis acicularis

\section{Common name}

biennial sagewort

western willow aster water arum (boreal spp. a range ext.)

northern willowherb

common mare's-tail

spiked water-milfoil

water smartweed

water smartweed

thread-leaved pondweed

various-leaved pondweed

clasping-leaf pondweed

silverweed

graceful cinquefoil

rough cinquefoil

yellow water crowfoot

western dock

arum-leaved arrowhead

marsh hedge-nettle

common dandelion

alsike clover

common bladderwort

rough hair grass

slough grass

narrow reed grass

water sedge

awned sedge

woolly sedge

graceful sedge

tufted hair grass

needle spike-rush 
Eleocharis palustris

Glyceria grandis

Hordeum jubatum

Juncus balticus

Juncus longistylis

Phleum pratense

Poa juncifolia

Poa palustris

Poa pratensis

Scirpus validus

Moss: $\quad$ Drepanocladus spp. creeping spike-rush

common tall manna grass

foxtail barley

wire rush

long-styled rush

timothy

alkali bluegrass

fowl bluegrass

Kentucky bluegrass

common great bulrush

brown moss

\subsection{Riparian}

The Riparian community type is a complex of stream channels and adjacent small terraces which occur along the named and un-named creeks and drainages in the study area. Many of these streams are intermittent. This unit is often associated with the Alluvial Fans/Floodplains community type which, where it occurs, is located adjacent to and upslope from the unit.

The unit is level to inclined, with slopes locally variable due to fluvial processes. Parent materials are fluvial in origin, with soil textures generally sandy clay loam. Soils are expected to be Regosolic. Drainage is variable, ranging from imperfectly drained on terraces to poorly drained in the immediate vicinity of stream channels. Ecological moisture regime varies from subxeric to submesic on terraces to hygric on creek beds. This broad ecological moisture regime gives rise to high plant species diversity.

Subhygric areas adjacent stream channels often support shrub communities which can consist of beaked willow (Salix bebbiana), yellow willow (S. lutea), common wild rose, buckbrush, red-osier dogwood (Cornus stolonifera) and silverberry (Elaeagnus commutata). 
In other areas, stream channel vegetation can be dominated by herbaceous vegetation such as water sedge (Carex aquatilis), graceful sedge (C. praegracilis), tufted hair grass (Deschampsia cespitosa), wire rush, creeping spike-rush (Eleocharis palustris), foxtail barley, slender wheat grass, wild mint (Mentha arvense) and seaside arrow-grass (Triglochin maritima). Submesic terraces can support shrub thickets of silverberry and common wild rose. Kentucky bluegrass is common in these areas, perhaps due to the grazing history of these preferred areas by cattle.

The diversity of shrub, forb and graminoid species found within these riparian areas give the vegetation of this community type a well-developed vertical structure not found in the majority of vegetation types comprising the Mixed Grass Ecoregion. As a result, this unit has great habitat value for many faunal species, including passerines, small mammals and ungulates, in the provision of food and cover. As well, northern leopard frogs, a "Red-listed" species in Alberta, were observed in this community type within Medicine Lodge Coulee during 1995 field investigations.

The following is a list of plants found within this community type based upon recorded data from 10 sites.

Species

Tree: $\quad$ Picea glauca (shrub height)

Shrub: $\quad$ Amelanchier alnifolia

Artemisia cana

Betula occidentalis

Elaeagnus commutata

Potentilla fruticosa

Rosa woodsii

Salix bebbiana

Salix lutea

Shepherdia canadensis

Spiraea betulifolia

Symphoricarpos occidentalis
Common name

white spruce

saskatoon

silver sagebrush

water birch

silverberry

shrubby cinquefoil

common wild rose

beaked willow

yellow willow

Canada buffaloberry

white meadowsweet

buckbrush 
Forb: $\quad$ Achillea millefolium Agoseris glauca Anemone canadensis Anemone cylindrica Anemone multifida Antennaria parvifolia Artemisia biennis Artemisia frigida Artemisia ludoviciana Aster ascendens Aster ericoides Aster falcatus Aster hesperius Aster laevis Astragalus dasyglottis Atriplex prostrata Campanula rotundifolia Chenopodium salinum

Cicuta maculata Cirsium arvense Cirsium flodmanii Cirsium vulgare Epilobium ciliatum Epilobium paniculatum Equisetum arvense Equisetum laevigatum Erigeron lonchophyllus Fragaria virginiana Galium boreale Gentiana affinis Geranium viscosissimum Geum aleppicum Geum triflorum Glaux maritima Glycyrrhiza lepidota Grindelia squarrosa Gutierrezia sarothrae Helianthus nuttallii Heterotheca villosa Hippuris vulgaris Lactuca biennis Linum lewisii common yarrow

yellow false dandelion

Canada anemone long-fruited anemone cut-leaved anemone small-leaved everlasting biennial sagewort pasture sagewort prairie sagewort western aster tufted white prairie aster creeping white prairie aster western willow aster smooth aster Drummond's milk vetch prostrate saltbush harebell oak-leaved goosefoot water-hemlock Canada thistle Flodman's thistle bull thistle northern willowherb annual willowherb common horsetail smooth scouring-rush hirsute fleabane wild strawberry northern bedstraw prairie gentian sticky purple geranium yellow avens three-flowered avens sea milkwort wild licorice gumweed broomweed common tall sunflower golden aster common mare's-tail tall blue lettuce wild blue flax 
Medicago sativa

Melilotus alba

Mentha arvensis

Monarda fistulosa

Plantago eriopoda

Plantago major

Polygonum arenastrum

Polygonum coccineum

Polygonum convolvulus

Potentilla anserina

Potentilla gracilis

Ranunculus cymbalaria

Ranunculus macounii

Ranunculus pensylvanicus

Rumex maritimus

Rumex occidentalis

Senecio eremophilus

Smilacina stellata

Solidago gigantea

Solidago rigida

Sonchus arvensis

Stachys palustris

Stellaria longifolia

Taraxacum officinale

Thalictrum venulosum

Thermopsis rhombifolia

Tragopogon dubius

Trifolium hybridum

Triglochin maritima

Urtica dioica

Vicia americana

Viola nephrophylla

Viola nuttallii

Zigadenus elegans

Zizia aptera

Graminoid: Agropyron repens

Agropyron smithii

Agropyron trachycaulum

Beckmannia syzigachne

Bouteloua gracilis

Bromus ciliatus alfalfa

white sweet-clover

wild mint

wild bergamot

saline plantain

common plantain

common knotweed

water smartweed

wild buckwheat

silverweed

graceful cinquefoil

seaside buttercup

Macoun's buttercup

bristly buttercup

golden dock

western dock

cut-leaved ragwort

star-flowered Solomon's-seal

low goldenrod

stiff goldenrod

perennial sow-thistle

marsh hedge-nettle

long-leaved chickweed

common dandelion

veiny meadow rue

golden bean

common goat's-beard

alsike clover

seaside arrow-grass

common nettle

wild vetch

bog violet

yellow prairie violet

white camas

heart-leaved Alexanders

quack grass

western wheat grass

slender wheat grass

slough grass

blue grama

fringed brome 
Bromus inermis

Calamagrostis stricta

Carex aquatilis

Carex praegracilis

Deschampsia cespitosa

Eleocharis palustris

Hordeum jubatum

Juncus balticus

Koeleria macrantha

Muhlenbergia richardsonis

Phleum pratense

Poa interior

Poa juncifolia

Poa palustris

Poa pratensis

Scirpus pallidus

Scirpus pungens

Spartina gracilis

Sphenopholis intermedia

Stipa curtiseta

Stipa viridula awnless brome

narrow reed grass

water sedge

graceful sedge

tufted hair grass

creeping spike-rush

foxtail barley

wire rush

June grass

mat muhly

timothy

inland bluegrass

alkali bluegrass

fowl bluegrass

Kentucky bluegrass

pale bulrush

three-square rush

alkali cord grass

slender wedge grass

western porcupine grass

green needle grass

\subsection{Shrub Draw}

The Shrub Draw community type occurs within ephemeral drainages which dissect many slopes within the study area. The unit can also include mesic, north-facing delayed snowmelt areas with extreme slopes (45 to 70 percent slope). Springs can occur within the draws which characterize the community type, usually discharging in the upper reaches of the draws. Ecological moisture regime is subhygric to submesic.

Shrubby cinquefoil (Potentilla fruticosa), common wild rose, buckbrush, wild red raspberry (Rubus idaeus) and round-leaved hawthorn (Crataegus rotundifolia) are the shrub species often found within these draws, commonly found in dense clumps. Shrub heights generally range between $0.5 \mathrm{~m}$ and $1.0 \mathrm{~m}$, though round-leaved hawthorn stands can reach heights up 
to 5 meters. Numerous forb species occur in this moist community type. Invader species such as Kentucky bluegrass and common dandelion (Taraxacum officinale) are common.

American pellitory (Parietaria pensylvanica), rare in Alberta, was found within the Shrub Draw community type in association with a mesic round-leaved hawthorn (Crataegus rotundifolia) and wild red raspberry (Rubus idaeus) thicket transitional between forest and grassland on north slope of the Cypress Hills, north-east of Reesor Lake during 1995 field investigations.

The following is a list of plants found within this community type based upon recorded data from three sites.

Species

Shrub: $\quad$ Amelanchier alnifolia Crataegus rotundifolia Juniperus horizontalis

Potentilla fruticosa

Prunus virginiana

Ribes oxyacanthoides

Rosa woodsii

Rubus idaeus

Salix bebbiana

Symphoricarpos occidentalis

Forb: $\quad$ Achillea millefolium Anemone cylindrica Anemone multifida

Antennaria parvifolia

Arctium minus

Artemisia ludoviciana

Aster falcatus

Aster hesperius

Aster laevis

Campanula rotundifolia

Cirsium arvense

Cirsium flodmanii
Common Name

saskatoon

round-leaved hawthorn

creeping juniper

shrubby cinquefoil

choke cherry

northern gooseberry

common wild rose

wild red raspberry

beaked willow

buckbrush

common yarrow

long-fruited anemone

cut-leaved anemone

small-leaved everlasting

common burdock

prairie sagewort

creeping white prairie aster

western willow aster

smooth aster

harebell

Canada thistle

Flodman's thistle 
Disporum trachycarpum

Equisetum laevigatum

Fragaria virginiana

Galium boreale

Geranium viscosissimum

Geum aleppicum

Geum triflorum

Glycyrrhiza lepidota

Hackelia americana

Helianthus nuttallii

Heracleum lanatum

Heuchera richardsonii

Hieraceum umbellatum

Lathyrus ochroleucus

Lithospermum ruderale

Lysimachia ciliata

Monarda fistulosa

Osmorhiza depauperata

Oxytropis sericea

Parietaria pensylvanica

Potentilla gracilis

Sanicula marilandica

Smilacina stellata

Solidago canadensis

Solidago gigantea

Solidago rigida

Taraxacum officinale

Thalictrum venulosum

Thermopsis rhombifolia

Trifolium hybridum

Urtica dioica

Vicia americana

Viola adunca

Viola canadensis

Viola nephrophylla

Zizia aptera

Graminoid: Agropyron trachycaulum

Agrostis scabra

Bromus ciliatus

Bromus inermis

Calamagrostis stricta fairybells

smooth scouring-rush

wild strawberry

northern bedstraw

sticky purple geranium

yellow avens

three-flowered avens

wild licorice

nodding stickseed

common tall sunflower

cow parsnip

Richardson's alumroot

narrow-leaved hawkweed

cream-colored vetchling

woolly gromwell

fringed loosestrife

wild bergamot

spreading sweet cicely

early yellow locoweed

American pellitory

graceful cinquefoil

snakeroot

star-flowered Solomon's-seal

Canada goldenrod

late goldenrod

stiff goldenrod

common dandelion

veiny meadow rue

golden bean

alsike clover

common nettle

wild vetch

early blue violet

western Canada violet

bog violet

heart-leaved Alexanders

slender wheat grass

rough hair grass

fringed brome

awnless brome

narrow reed grass 
Carex deweyana

Carex sprengelii

Danthonia californica

Glyceria striata

Juncus balticus

Phleum pratense

Poa palustris

Poa pratensis

Sphenopholis intermedia

Stipa viridula
Dewey's sedge

Sprengel's sedge

California oat grass

fowl manna grass

wire rush

timothy

fowl bluegrass

Kentucky bluegrass

slender wedge grass

green needle grass

The prevalence of important browse species and the shelter provided by the draws, gives this community type relatively significant habitat value within the surrounding grassland areas.

\subsection{Forest}

Forest community types within the Cypress Hills in general are concentrated at higher elevations on the northern and western slopes of the hills. The occurrence of these forests are largely attributed to the climatic effects of the Cypress Hills (Holmes 1970 and 1969, Newsome and Dix 1968, Breitung 1954). Transitions between forest and grassland is usually quite sharp, though tree and shrub communities can extend into the grasslands at lower elevations along ravines and coulees where moisture conditions are favourable due to snow accumulation and springs (Holmes 1970).

Forest communities in the Cypress Hills were studied in detail by Newsome and Dix (1968) and found to have greater similarity to western cordilleran rather than boreal forests, based on the dominant tree species and their understorey components. Dominant tree species in the Cypress Hills include lodgepole pine (Pinus contorta var. latifolia), aspen (Populus tremuloides) and white spruce (Picea glauca). Aspen is widespread throughout the Cypress Hills (Newsome and Dix 1968). White spruce is favoured in cool moist habitats such as at stream margins, spring areas and at the base of north-facing slopes. Lodgepole pine is common at higher elevations. 
Three forest community types were delineated within the Eagle Butte Sensitive Area, namely Deciduous Forest, Coniferous Forest and Mixed Wood. The community types are quite general due to the low level of survey intensity possible within forested areas for this study. These community types are described below. All three community types occur on similar terrain as the Coulee Slopes and Eroding Slopes community types, but at higher elevations.

\subsubsection{Deciduous Forest}

The Deciduous Forest community type consists of forest stands dominated by deciduous tree species, generally aspen. Topography is generally inclined to ridged, with pronounced relief. Slopes are often strong (16 to 30 percent slope) to extreme (45 to 70 percent slope). Ecological moisture regime is mesic, becoming subhygric at lower slope positions. Soil textures are generally sandy loam, but can be clay loam on subhygric sites. Drainage is well to moderately well.

Aspen is the dominant tree species throughout much of the community type, though subhygric sites can be dominated by balsam poplar (Populus balsamifera). White spruce regeneration can be found in the understorey. Common understorey shrubs under aspen include saskatoon, prickly rose (Rosa acicularis) and snowberry (Symphoricarpos albus). Numerous additional shrub species are often present in lesser occurrences. Common forbs include wild strawberry (Fragaria virginiana), thin-leaved ragwort (Senecio pseudaureus), smooth aster (Aster laevis), western Canada violet (Viola canadensis) and wild white geranium (Geranium richardsonii), to name a few. Grasses commonly include purple oat grass (Schizachne purpurascens), slender wheat grass, drooping wood-reed (Cinna latifolia) and bluejoint (Calamagrostis canadensis).

Stands dominated by balsam poplar can be found in valley bottoms on subhygric sites with fine-textured soils. Associated species can include Manitoba maple (Acer negundo), wild 
black currant (Ribes americanum), wild red raspberry, common horsetail (Equisetum arvense), late goldenrod (Solidago gigantea), western Canada violet, cow parsnip (Heracleum lanatum), slender wheat grass, bluejoint and awnless brome. Of particular note is the occurrence of tall meadow rue (Thalictrum dasycarpum), rare in Alberta, at such sites.

The following is a list of plants found within this community type based upon recorded data from four sites.

\section{Species}

Tree: $\quad$ Acer negundo

Populus balsamifera

Populus tremuloides

Picea glauca (regeneration)

Shrub: $\quad$ Amelanchier alnifolia

Clematis occidentalis

Cornus stolonifera

Crataegus rotundifolia

Elaeagnus commutata

Juniperus communis

Prunus pensylvanica

Prunus virginiana

Ribes americanum

Ribes oxyacanthoides

Rosa acicularis

Rubus idaeus

Rubus pubescens

Salix bebbiana

Spiraea betulifolia

Symphoricarpos albus

Symphoricarpos occidentalis

Vaccinium caespitosum

Forb: $\quad$ Actaea rubra

Anemone canadensis

Antennaria parvifolia

Arctostaphylos uva-ursi

Aster ciliolatus
Common Name

Manitoba maple

balsam poplar

aspen

white spruce

saskatoon

purple clematis

red-osier dogwood

round-leaved hawthorn

silverberry

ground juniper

pin cherry

choke cherry

wild black currant

northern gooseberry

prickly rose

wild red raspberry

dewberry

beaked willow

white meadowsweet

snowberry

buckbrush

dwarf bilberry

baneberry

Canada anemone

small-leaved everlasting

common bearberry

Lindley's aster 
Aster conspicuus

Aster laevis

Campanula rotundifolia

Disporum trachycarpum

Epilobium angustifolium

Equisetum arvense

Fragaria virginiana

Galium boreale

Galium triflorum

Geranium richardsonii

Heracleum lanatum

Lathyrus ochroleucus

Moehringia lateriflora

Monarda fistulosa

Orthilia secunda

Osmorhiza depauperata

Pyrola asarifolia

Ranunculus acris

Sanicula marilandica

Senecio pseudaureus

Smilacina stellata

Solidago gigantea

Stachys palustris

Taraxacum officinale

Thalictrum dasycarpum

Thalictrum venulosum

Trifolium hybridum

Urtica dioica

Vicia americana

Viola adunca

Viola canadensis

Viola nuttallii

Graminoid: Agrohordeum macounii

Agropyron trachycaulum

Bromus carinatus

Bromus ciliatus

Bromus inermis

Calamagrostis canadensis

Carex siccata

Cinna latifolia

Elymus glaucus showy aster

smooth aster

harebell

fairybells

common fireweed

common horsetail

wild strawberry

northern bedstraw

sweet-scented bedstraw

wild white geranium

cow parsnip

cream-colored vetchling

blunt-leaved sandwort

wild bergamot

one-sided wintergreen

spreading sweet cicely

common pink wintergreen

tall buttercup

snakeroot

thin-leaved ragwort

star-flowered Solomon's-seal

late goldenrod

marsh hedge-nettle

common dandelion

tall meadow rue

veiny meadow rue

alsike clover

common nettle

wild vetch

early blue violet

western Canada violet

yellow prairie violet

Macoun's wild rye

slender wheat grass

keeled brome

fringed brome

awnless brome

bluejoint

hay sedge

drooping wood-reed

smooth wild rye 
Festuca saximontana

Poa interior

Poa pratensis

Schizachne purpurascens
Rocky Mountain fescue inland bluegrass Kentucky bluegrass purple oat grass

\section{$\underline{4.10 .2}$ Coniferous Forest}

The Coniferous Forest community type consists of forest stands with a tree canopy essentially comprised exclusively of lodgepole pine. Such sites tend to be subxeric, well drained, northfacing mid-and upper-slopes at high elevations in the vicinity of the Cypress Hills Plateau. Soil textures are often sandy clay loam. Topography is generally inclined to ridged, with pronounced relief.

Vertical structure is poorly developed, with shrub layers almost non-existent. Forb cover is not extensive, and the number of species present is relatively few. Pine reed grass (Calamagrostis rubescens) is the dominant grass. Pine-drops (Pterospora andromeda), rare in Alberta, can be found in these stands.

The following is a list of plants found within this community type based upon recorded data from one site.

\section{$\underline{\text { Species }}$}

Tree: $\quad$ Pinus contorta

Shrub: $\quad$ Chimaphila umbellata

Rosa acicularis

Spiraea betulifolia

Forb: $\quad$ Arnica cordifolia

Aster conspicuus

Hieracium albiflorum

Lathyrus ochroleucus

Osmorhiza depauperata

Polygonum spp.

\section{Common Name}

lodgepole pine

prince's-pine

prickly rose

white meadowsweet

heart-leaved arnica

showy aster

white hawkweed

cream-colored vetchling

spreading sweet cicely

knotweed 
Pterospora andromedea

Pyrola chlorantha

Thalictrum venulosum

Graminoid: Calamagrostis rubescens

Oryzopsis asperifolia

Moss: $\quad$ Pleurozium schreberi

Ptilium crista-castrensis

Lichen:

Cladonia spp. pine-drops

greenish-flowered wintergreen

veiny meadow rue

pine reed grass

white-grained mountain rice grass

Schreber's moss

knight's plume moss

lichen

\subsubsection{Mixed Wood}

The Mixed Wood community type is the most extensive forest community type mapped in the study area. The community type consists of aspen- or conifer-dominated mixed wood forest with inclusions of pure deciduous or pure conifer stands. Topography is generally inclined to ridged, with pronounced relief. Slopes are often strong (16 to 30 percent slope) to extreme (45 to 70 percent slope). Ecological moisture regime is mesic, becoming subhygric at lower slope positions. Soil textures are generally sandy loam, but can be clay loam on subhygric sites. Drainage is well to moderately well.

White spruce is an important component of this community type, often co-dominant with aspen. Previous researchers thirty to forty years ago expected white spruce to succeed pioneer species such as lodgepole pine in the absence of disturbance (Newsome and Dix 1968, Breitung 1954). Fire has been strictly controlled during this time, and white spruce now appears to be a common conifer in the study area.

Vertical structure of mixed wood stands are generally well developed, with tall shrub, low shrub, forb and graminoid strata well represented. Understorey woody vegetation can include regenerating white spruce, saskatoon, snowberry, white meadowsweet (Spiraea 
betulifolia), Canada buffaloberry (Shepherdia canadensis), northern gooseberry (Ribes oxyacanthoides) and numerous other shrub species. Numerous forb species occur, including western Canada violet, northern bedstraw, wild strawberry, cow parsnip, various asters, baneberry (Actaea rubra), cream-colored vetchling (Lathyrus ochroleucus) and wild vetch (Vicia americana), among others. Grass species can include smooth wild rye (Elymus glaucus), slender wheat grass, white-grained mountain rice grass (Oryzopsis asperifolia), purple oat grass and bluejoint, to name a few.

The following is a list of plants found within this community type based upon recorded data from 14 sites.

\section{$\underline{\text { Species }}$}

Tree: $\quad$ Picea glauca

Pinus contorta

Populus balsamifera

Populus tremuloides

Shrub: $\quad$ Amelanchier alnifolia

Arctostaphylos uva-ursi

Clematis occidentalis

Cornus stolonifera

Crataegus douglasii

Crataegus rotundifolia

Elaeagnus commutata

Juniperus communis

Prunus pensylvanica

Prunus virginiana

Ribes oxyacanthoides

Rosa acicularis

Rubus idaeus

Salix bebbiana

Shepherdia canadensis

Spiraea betulifolia

Symphoricarpos albus

Symphoricarpos occidentalis

Vaccinium caespitosum

Viburnum edule

\section{Common Name}

white spruce

lodgepole pine

balsam poplar

aspen

saskatoon

common bearberry

purple clematis

red-osier dogwood

Douglas hawthorn

round-leaved hawthorn

silverberry

ground juniper

pin cherry

choke cherry

northern gooseberry

prickly rose

wild red raspberry

beaked willow

Canada buffaloberry

white meadowsweet

snowberry

buckbrush

dwarf bilberry

low-bush cranberry 
Forb: $\quad$ Achillea millefolium

Actaea rubra

Anemone canadensis

Antennaria neglecta

Artemisia ludoviciana

Aster ciliolatus

Aster conspicuus

Aster laevis

Campanula rotundifolia

Cerastium arvense

Chenopodium gigantospermum

Cirsium vulgare

Corallorhiza maculata

Cornus canadensis

Disporum trachycarpum

Epilobium angustifolium

Epilobium ciliatum

Equisetum arvense

Fragaria virginiana

Galeopsis tetrahit

Galium boreale

Galium triflorum

Gentianella amarella

Geranium richardsonii

Geum aleppicum

Hedysarum boreale

Hieracium albiflorum

Hieracium umbellatum

Heracleum lanatum

Lactuca pulchella

Lathyrus ochroleucus

Linnaea borealis

Lithospermum ruderale

Medicago sativa

Moehringia lateriflora

Monarda fistulosa

Osmorhiza depauperata

Orthilia secunda

Parietaria pensylvanica

Pyrola asarifolia

Ranunculus acris

Sanicula marilandica common yarrow

baneberry

Canada anemone

broad-leaved everlasting

prairie sagewort

Lindley's aster

showy aster

smooth aster

harebell

field mouse-ear chickweed

maple-leaved goosefoot

bull thistle

spotted coralroot

bunchberry

fairybells

common fireweed

northern willowherb

common horsetail

wild strawberry

hemp-nettle

northern bedstraw

sweet-scented bedstraw

felwort

wild white geranium

yellow avens

northern hedysarum

white hawkweed

narrow-leaved hawkweed

cow parsnip

common blue lettuce

cream-colored vetchling

twinflower

woolly gromwell

alfalfa

blunt-leaved sandwort

wild bergamot

spreading sweet cicely

one-sided wintergreen

American pellitory

common pink wintergreen

tall buttercup

snakeroot 
Senecio eremophilus

Smilacina racemosa

Smilacina stellata

Solidago gigantea

Taraxacum officinale

Thalictrum venulosum

Trifolium hybridum

Urtica dioica

Vicia americana

Viola adunca

Viola canadensis

Graminoid: Agropyron trachycaulum

Agrostis scabra

Bromus ciliatus

Calamagrostis canadensis

Calamagrostis rubescens

Carex deweyana

Carex rostrata

Carex sprengelii

Cinna latifolia

Elymus glaucus

Festuca scabrella

Oryzopsis asperifolia

Phleum pratense

Poa compressa

Poa interior

Poa pratense

Schizachne purpurascens cut-leaved ragwort

false Solomon's-seal

star-flowered Solomon's-seal

late goldenrod

common dandelion

veiny meadow rue

alsike clover

common nettle

wild vetch

early blue violet

western Canada violet

slender wheat grass

rough hair grass

fringed brome

bluejoint

pine reed grass

Dewey's sedge

beaked sedge

Sprengel's sedge

drooping wood-reed

smooth wild rye

rough fescue

white-grained mountain rice grass

timothy

Canada bluegrass

inland bluegrass

Kentucky bluegrass

purple oat grass

squawroot$$
\text { squawroot }
$$

Lichen: Perideridia gairdneri

\subsection{Agricultural}

The Agricultural community type comprises sites within the study area where native plant communities have been compromised by agricultural activities. Such sites include those currently under cultivation for annual crops, hay, or improved pasture areas seeded to non- 
native forage species. Previously cultivated lands which are now reverting to native prairie have also been included where mappable. 


\subsection{SLOPE CLASSES}

Slopes within the study area are highly variable and consist primarily of complex slopes, with minor areas of simple slopes. Simple slopes are slopes which are relatively uniform, such as those found on alluvial fans or on upland areas with level to undulating topography. In contrast, complex slopes encompass a relatively wide variation of slope gradients within them, such as areas dissected by streams, areas of slumping, bedrock controlled uplands or hummocky topography.

Within the Eagle Butte Sensitive Area, areas largely consisting of simple slopes include the Alluvial Fan\Floodplain community type, such as found in the Manyberries Creek valley and Medicine Lodge Coulee (Map 2). As well, a relatively extensive area of simple slopes can be found where the Native Grassland community type occurs on the Cypress Hills Plateau in portions of the study area south of Cypress Hills Provincial Park. The Ephemeral Wetland community type also consists of simple slopes. Topography within these community types is relatively subdued, with slopes generally ranging from level ( 0 to 0.5 percent slope) to gentle (6 to 9 percent slope).

The Eagle Butte Sensitive Area predominantly consists of extensive areas with strong to rugged topography. Complex slope classes presented on Map 2 serve to give a relative indication of the topographic complexity of the community types associated with these complex slope classes. Much of the upland areas in western portions of the study area are bedrock controlled, exhibiting rolling to ridged topography. Slope polygons in these areas incorporate numerous slope classes, with complex slopes usually spanning a wide range of slope classes. Slope classes often range from very gentle ( 2 to 5 percent slope), gentle (6 to 9 percent) or moderate (10 to 15 percent slope) with subdominant slopes often very strong (31 to 45 percent slope). The eroding and slumping slopes common on the flanks of the 
Cypress Hills, Medicine Lodge Coulee, Manyberries Creek valley and the many tributary drainages of these areas consist of complex slopes ranging from moderate (16 to 30 percent slope) to steep (71 to 100 percent). The Eroding Slopes and Shrub Draw community types possess the strongest relief in the study area, with slopes generally ranging from extreme (45 to 70 percent slope) to steep, with some eroding slope areas having very steep slopes (greater than 100 percent). Complex slopes are also prevalent within the Hummocky Grassland community type in the northern portion of the study area. Slopes here range from moderate to strong, becoming relatively more subdued in northeastern portions of the study area, with slopes ranging from gentle to moderate. In addition, the Riparian community type possesses complex slopes due to the many small fans, terraces and meander scars found within these areas.

Slope gradient and complexity have implications with regard to vegetation community establishment in terms of their effects on moisture regimes and on the intensity of aspect effects, both factors which affect, in part, the type of vegetation community found at a given site. Section 4.0 of this report discusses this in more detail. Implications also exist with regard to the potential for erosion to occur within a given unit, which is a factor influencing the inherent sensitivity of an area to disturbance and which is discussed in Section 7.0. 


\subsection{SIGNIFICANT FEATURES}

Environmentally significant areas (ESAs) are areas which are deemed to be important to society in terms of ensuring the maintenance of ecological processes or by the provision of useful products. As well, these areas are often sensitive to disturbance (Wallis 1991).

The Eagle Butte Sensitive Area lies almost entirely within portions of three different ESAs as identified by Wallis (1991). The Cypress Hills ESA was designated as nationally significant, the Eagle Butte ESA was designated as provincially significant, and the Lodge Creek ESA was classed as regionally significant.

The Cypress Hills ESA includes those portions of the study area north and east of Medicine Lodge Coulee, extending to the Alberta-Saskatchewan border. Significant features identified by Wallis (1991) for the ESA include the unglaciated Cypress Hills Plateau, rough fescue grasslands similar to those found in the foothills of southwestern Alberta, relict montane forests, fresh water springs, extensive marshes and permanent wetlands, numerous rare and disjunct plant and animal species such as flowering quillwort (Lilaea scilloides) and northern leopard frog, breeding bird diversity, key moose, elk and deer habitat, sharp-tailed grouse dancing grounds and snake hibernacula.

The Eagle Butte ESA includes the western portion of the study area. Significant features identified by Wallis (1991) for this ESA include foothills fescue grasslands, relict montane forests, wetlands and riparian shrub areas, rare species such as flowering quillwort (Lilaea scilloides) and numerous disjunct plant and bird species, sharp-tailed grouse dancing grounds and key moose, elk and deer habitat. 
The Lodge Creek ESA extends into the study area within the Lodge Creek portion of Medicine Lodge Coulee. This ESA was considered significant by Wallis (1991) due to its extensive riparian habitats, rugged grassland and badland topography, grouse dancing grounds, breeding bird diversity, and the fact that it is part of the largest and most diverse mixed grassland area in Canada.

Significant features of the Eagle Butte Sensitive Area are summarized below. Significant areas or sites or areas deserving special mention are also identified here, with a detailed discussion of each being presented in Section 9.0 and in Map 3.

\subsection{Significant Geological/Landform Features}

The most significant geological or landform feature within the study area is the Cypress Hills, which have a profound effect on the local climate, topographic diversity, geologic diversity, hydrogeology and their collective effects on plant and animal habitat diversity in the area.

The Cypress Hills Plateau remained unglaciated during the Laurentide glacial advances, is capped with Tertiary conglomerate, the Cypress Hills Formation, which provides economically important gravel resources which have been exploited to some degree. Lithologically similar gravels occur on Eagle Butte (Westgate 1968). Precipitation falling on the plateau percolates through the conglomerate, becoming groundwater which discharges as springs along the flanks of the Cypress Hills (Roed n.d.).

The rapid elevational relief created by the Cypress Hills have created an "oasis effect" on the local climate, as described by Holmes $(1970,1969)$. Increased moisture regimes under the local climate of the Cypress Hills support disjunct vegetation communities including rough fescue-dominated grasslands and forests which are more typical of areas hundreds of miles 
distant, separated from the Cypress Hills populations by semi-arid Mixed Grass Prairie of the Northern Great Plains.

Numerous bedrock formations are exposed along the flanks of the Cypress Hills within the study area, providing geologic diversity. As well, the Whitemud Formation provides economically important clays used in industrial ceramic production.

\subsection{Significant Hydrological Features}

Hydrological features of significance within Eagle Butte Sensitive Area include springs of good quality potable water, riparian areas, wetlands and Elkwater Lake.

Numerous fresh water springs discharge along the flanks of the Cypress Hills, with groundwater recharged from precipitation falling on the Cypress Hills Plateau and percolating through the conglomerate of the Cypress Hill Formation which caps the plateau (Roed n.d.). As such, development activities on the Cypress Hills Plateau could have implications with regard to their effect on groundwater quality. The springs include two major springs with measurable flow rates mapped in the study area by Borneuf (1976). One major spring is located in Section 31-Twp. 8-Rge. 4-W4M has been measured at a flow rate of 25 imperial gallons per minute (114 litres per minute), while a major spring in Section 21-Twp.7-Rge. 3W4M has been measured at a flow rate of 9 imperial gallons per minute (41 litres per minute) (Borneuf 1976). Major springs with a measurable flow rate are rare, even in the Cypress Hills. As well, major and minor springs along the Cypress Hill provide habitat for numerous rare or uncommon plant and animal species (Wallis 1990, Ecoplans Ltd. n.d., Biota Consultants Ltd. and Komex International 1992). 
Riparian areas within the study area provide valuable wildlife habitat. Most streams in the study area are intermittent, however, northern leopard frogs were observed in Lodge Creek and in an unnamed drainage to Michel Reservoir during 1995 field investigations. As well, many drainages dissecting the flanks of the Cypress Hills have been dammed by man for water developments, and by beaver. Ponds created by beaver dams in the study area provide important habitat for northern leopard frog populations, providing water deep enough to enable the frogs to overwinter (Biota Consultants Ltd. and Komex International 1992, Wallis 1990). As well, two western painted turtles, endangered in Alberta, were reported in a spring-fed beaver pond within the Eagle's Nest Candidate Natural Area by previous researchers (Biota Consultants Ltd. and Komex International 1992).

Numerous permanent and ephemeral wetlands occur in the study area as a result of beaver damming and man-made reservoir development, as described above, and as a result of the "knob-and-kettle" topography created by the hummocky disintegration moraine located in the northern portion of the study area. Many of these wetlands are valuable in providing habitat for rare and uncommon fauna, such as described above, and for rare and uncommon flora (Wallis 1990, Biota Consultants Ltd. and Komex International 1992, Ecoplans Ltd n.d.). Ephemeral and permanent wetlands in hummocky moraine areas within the sensitive area also provide significant waterfowl and shorebird staging habitats.

The western tip of Elkwater Lake extends into the study area. This lake is spring-fed and is notable as one of the few permanent, major fresh water lakes in southeastern Alberta. Elkwater Lake provides valuable waterfowl habitat, and it also is a major recreational resource for the region. 


\subsection{Significant Archaeological/Palaeontological Features}

The Cypress Hills played an important role in prehistoric and historic human culture (Bonnichsen and Baldwin 1978). During prehistoric time, the Cypress Hills likely were important camping areas due to the relatively greater diversity of resources within a small geographical area, a paramount consideration to the pedestrian hunter-gatherer prehistoric peoples. Native peoples of historic time utilized horses for transportation, particularly during the late historic period dating from approximately A.D. 1800 to 1885, but the Cypress Hills continued to play an important role to the indigenous people of the region. Activities associated with the Cypress Hills during this time included the use of the Cypress Hills for wintering areas, hunting, plant collecting, war scouting, Sun Dance ceremonies and burial sites. As a result, numerous archaeological sites have been found within the Cypress Hills, with 64 sites found to date within Eagle Butte Sensitive Area (Alberta Culture files). Campsites are especially numerous. Other features include tipi rings, effigies, cairns, stone circles, stone rings and scatter sites. The Eagle Butte Sensitive Area lies within an archaeological resource sensitivity zone rated as "High" (M. Woodhouse, pers. comm.).

Historic sites located within the study area include the site of the Medicine Lodge N.W.M.P. post in Medicine Lodge Coulee near Eagle Butte, established in 1894 and abandoned in 1906 (Alberta Culture files). As well, two old coal mines occur in the study area, with one abandoned in 1924 and the other abandoned in 1943.

Fossil leaves from deciduous tree species commonly occur in the Ravenscrag Formation, with fossils of invertebrates such as pelecypods and gastropods found in older beds in the formation (Roed n.d.). The Frenchman Formation is locally rich in vertebrate fauna, including dinosaur species which existed immediately prior to the extinction of dinosaurs 62 million years ago. 
Site specific investigations are currently conducted in conjunction with the referral process for petroleum development applications (D. Wetzel, pers. comm.), and a referral to Resource Management Program Unit, Cultural Facilities and Historical Resources Division, Alberta Community Development would be prudent prior to site-specific development activities in the study area.

\subsection{Significant Flora}

Determination of rare flora occurrences is best done by intensive vegetation survey. Project constraints precluded this. The following discussion is based on the results of 1995 field work within the study area and on literature review.

The unique topographic and geologic features of the Eagle Butte Sensitive Area have, in many cases, led to the occurrence of a greater number of rare plant species than is generally found in the Mixed Prairie. The Eagle Butte Sensitive Area may contain up to 25 species of rare or uncommon plants, many of which were confirmed in the study area during 1995 field work or by previous researchers (Appendix A). Of these, seven species are considered as rare in Canada, 11 species are rare in Alberta, three species have restricted or local distribution in Alberta, and three species can be considered as range extensions (Argus and Pryer 1990, Packer and Bradley 1984, Wallis 1990, Moss 1983, Wallis et al. 1987).

A species list of significant flora as described above that were identified in, or likely to be found in, the Eagle Butte Sensitive Area is presented in Appendix A.

\subsection{Significant Fauna}


For the purpose of this report, wildlife species will be considered as significant if they have:

(a) been designated as such by the Committee on the Status of Endangered Wildlife in Canada (COSEWIC 1995);

(b) been designated as such by Alberta Natural Resources Service (AFWD 1991);

(c) been cited as being locally rare or uncommon in the province; or

(d) been cited as being of relatively high abundance in the Eagle Butte Sensitive Area.

COSEWIC determines the national status of wild species, sub-species, and separate populations in Canada. All native fish, amphibians, reptiles, birds, and mammals are included; invertebrate animals are not. COSEWIC has identified five risk categories for Canadian wildlife species, as follows (COSEWIC 1995):

Extinct - A species formerly indigenous to Canada that no longer exists anywhere.

Extirpated- A species no longer existing in the wild in Canada but existing elsewhere.

Endangered - A species threatened with imminent extinction or extirpation throughout all or a significant portion of its Canadian range.

Threatened - A species likely to become endangered in Canada if the factors affecting its vulnerability are not reversed. 
Vulnerable - A species particularly at risk because of low or declining populations, small range, or for some other reason, but not a threatened species.

Provincial populations of wildlife species in Alberta have also been evaluated by Alberta Natural Resources Service (AFWD 1991) and their status has been determined and classified as follows:

Red - species whose populations are nonviable or at immediate risk of declining to non-viable levels in Alberta. They have, or will be considered for, designation as Endangered Species in Alberta.

Blue - species which are also at risk but the threats they face are less immediate. Particularly vulnerable to noncyclical declines in population or habitat, or to reductions in provincial distribution.

Yellow - sensitive species which are not at risk. They may require special management to address concerns related to low natural populations, limited provincial distribution, or particular biological characteristics.

Green-species not considered to be at risk. Their populations are healthy and often widespread and key habitats are generally secure.

Based on the aforementioned criteria, the following species or species assemblages have been identified as being significant in the Eagle Butte Sensitive Area. 


\subsubsection{Ungulates}

Ungulates are likely the most well-known mammalian species occuring in the study area. The present ungulate assemblage in the Eagle Butte and Cypress Hills region is considerably depauperate relative to historical ungulate habitation. Prior to their extirpation from the Canadian prairies, plains bison (Bison bison athabascae) were reported to frequent the Cypress Hills and Dickinson (1991) identified wallow sites that are still visible on the benchlands. Pronghorn antelope (Antilocapra americana) also suffered a fate similar to plains bison on the prairies. The 19th century naturalist Ernest Thompson Seton estimated a North American antelope population of 45,000,000 in 1868 - a population which, by the early 1900s, verged on extinction. Advancing settlement across western Canada, coupled with overhunting and severe winter weather, contributed to the decline of the species over most of its range.

The recovery of the pronghorn antelope was spurred in southern Alberta and Saskatchewan by the move to set aside reserves, or antelope parks, where hunting and other disturbances were prohibited. Three such reserves were established as legislated National Parks in areas surrounding the Cypress Hills - Nemiskam National Park and Wawaskesy National Park in Alberta and Menissawok National Park in Saskatchewan. Pronghorn antelope populations increased under sanctuary conditions and, therefore, all three national parks were eventually abolished, having "performed their role and outlived their usefulness" (Lothian 1987). Menissawok National Park was abolished by Orders-in-Council in 1930, Wawaskesy in 1935, and Nemiskam in 1947. Presently, pronghorn antelope do not make extensive use of open grasslands in the Eagle Butte area but individual stragglers have been recorded in plateau grasslands in summer (Dickinson 1991). 
Four species of ungulates - white-tailed deer, mule deer, elk, and moose - now occur regularly in the Cypress Hills, with elk and moose being largely limited to forested uplands and wooded, riparian habitats. Both deer species, although exhibiting partitioned habitat preferences in some instances, occur over much more of the study area, as they occupy open grasslands and shrublands as well. Ungulate use of appropriate habitats in the Eagle Butte Sensitive Area has not been adequately assessed to date, however, intermittent information has been collected by Alberta Natural Resources Service (formerly Alberta Fish and Wildlife Division and Alberta Parks Service) on elk, moose, and deer in Cypress Hills.

Elk were extirpated from the study area around the turn of the century, with the last elk being shot in the Saskatchewan portion of the Cypress Hills in 1909. They were, subsequently, reintroduced to the Hills in 1937 and recent surveys conducted by Alberta Fish and Wildlife (Gudmundson 1995) reveal populations of approximately 350 elk in the Alberta portion of the Cypress Hills and adjacent areas. Gunson (1992) estimates a late winter provincial population of 12,840 elk on provincial lands and an additional 4,100 elk wintering in Waterton Lakes, Banff, Jasper, and Elk Island national parks.

Because elk are widely recognized as an adaptable and opportunistic species, it is difficult to specify their habitat requirements over a broad geographic area. Elk distribution and habitat selection in the Eagle Butte Sensitive Area is determined by a combination of many factors including topography, vegetation structure, forage quality and quantity, tradition and habitual behaviour, weather conditions, predators, and human activities. The primary characteristic of elk habitat is the requirement for interspersion of forage, escape, and cover resources. Foraging areas for elk are usually in the open, thermal cover is best provided by coniferous forested stands, and dense forests with well-developed understories provide optimal predator relief. Thus, elk are an ecotonal, or multitypic, species, favoring high 
contrast forest edges which provide an abundance of forage in close proximity to escape and thermal cover.

Food habits of elk have been extensively reviewed for foothill and northern forest habitats (see Morgantini and Russell 1983, Nietfeld 1983, Fargey and Hawley 1989, and Renecker and Hudson 1992) but relatively little is known about their food habits in the Cypress Hills and Eagle Butte areas, save Dickinson (1969), Keith (1977), Lee (1979), and Hunt and Wiltse (1980). The diets of elk are extremely variable and largely dependent upon local forage availability. While Kufeld (1973) found that 159 forbs, 59 grasses, and 95 shrubs have been reported as elk forage, Lee (1979) found that, in the Cypress Hills, grasses are preferred forage in biomass throughout the year and are consumed in both succulent and dry seasons (Table 2).

Snow depth and condition are major determining factors of elk diets on winter ranges. Skovlin (1982) refers to snow depth as the factor most limiting to elk distribution and movement, as snow depths of 41 to $71 \mathrm{~cm}$ have caused elk to switch from grazing to browsing, and depths of $76 \mathrm{~cm}$ are considered detrimental to travel and movement (Nietfeld et al. 1984). In response to hunting patterns and variable environmental conditions, elk in the Eagle Butte Sensitive Area exhibit a migratory pattern, linking the winter, intermediate, and summer ranges together. Such spring and summer altitudinal movements are closely tied to the tracking of palatable plant phenology while the fall migration to lower altitudes is a response to snow accumulations at higher elevations. Therefore, sites protected by topography or vegetation from strong winds, snow crusting, and snow drifting are selected in winter and early spring. In the Eagle Butte Sensitive Area, major wintering areas for elk include the Reesor Hills (north of Reesor Lake and Battle Creek), the hills immediately north of Spruce Coulee Reservoir, and the east-facing slopes surrounding Medicine Lodge Coulee and Creek. 


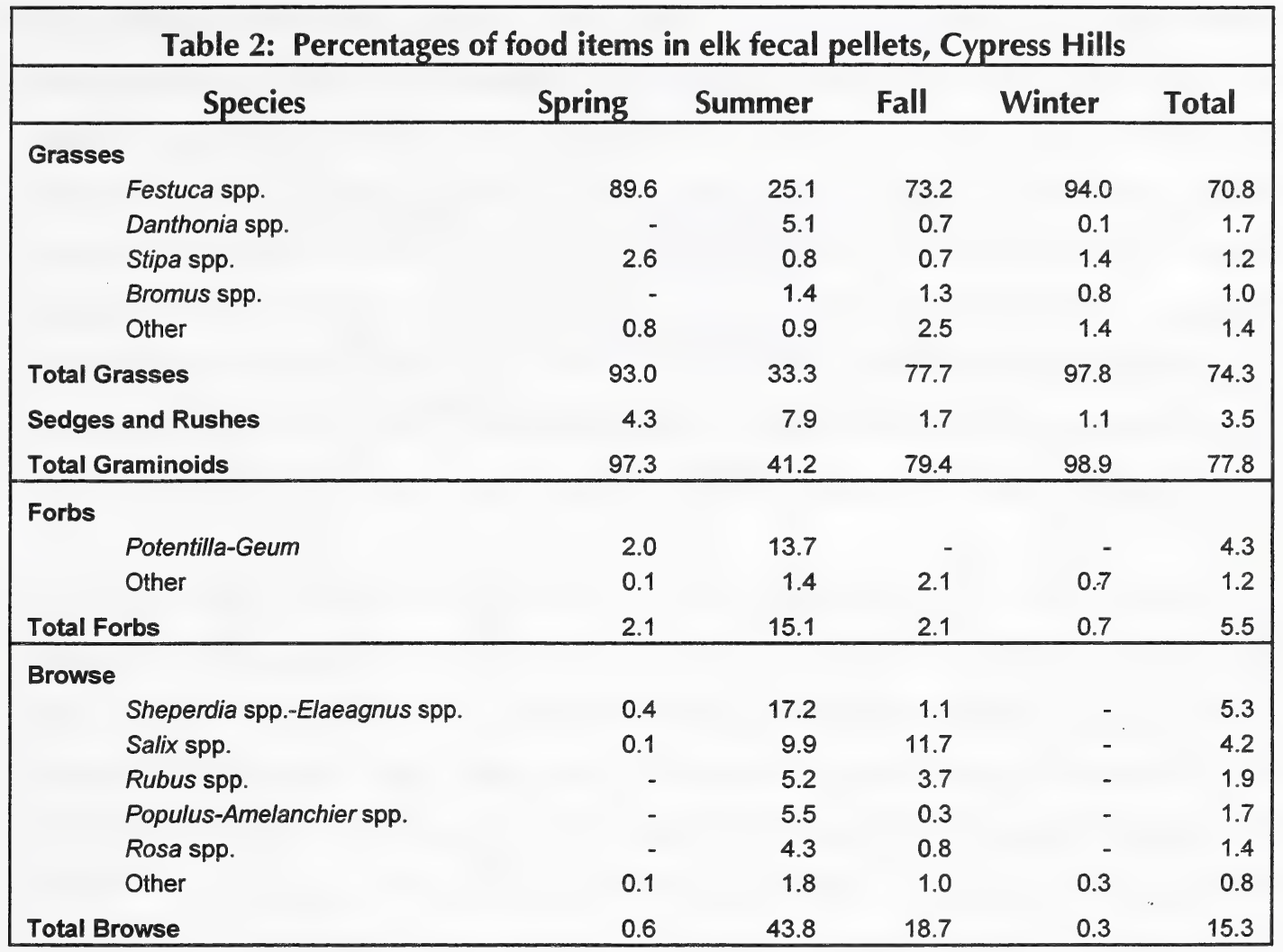

Commonly noted as a species of the northern boreal forest, moose also occur in the Eagle Butte Sensitive Area as a disjunct population. The distribution of moose throughout their range is closely related to the range of northern trees and shrubs. The presence of relict boreal and cordilleran plant species in the Eagle Butte and Cypress Hills area, however, has attracted a relict fauna as well, one of which is the moose. Dickinson (1968) cited historical accounts that moose antlers were found in the Cypress Hills in the early 1900s and other unconfirmed reports that moose were in the area prior to European settlement. There exists, however, considerable debate as to whether or not moose were indigenous to the Cypress Hills area. If the species was native to the Hills, it was very rare and was extirpated by the 
1920s. In 1956, two female and two male moose were translocated from Elk Island National Park and released in Cypress Hills immediately east of Elkwater Lake and subsequently spread throughout the area. Ressler (n.d.) gives a present population estimate of between 120 and 150 moose in Cypress Hills Provincial Park, although aerial survey results synthesized by Alberta Fish and Wildlife Division (Gudmundson 1993) indicate that this may be an overestimation. The Fish and Wildlife Division has found totals of 27 to 53 moose in their survey area, which includes portions of the Cypress Hills and Eagle Butte areas. Smith (1993) also reports that local residents have cited moose populations to have severely declined in the Eagle Butte area and that the species is now very rarely observed.

Moose eat a variety of plants, ranging from mosses to tree bark, but this forage diversity is greatly reduced in winter, when they must feed only on woody browse that extends above the snow. Coady (1982) identifies the availability of these winter forage species as one of the major limiting factors of moose populations throughout their range. The moose populations in the Eagle Butte Sensitive Area and in Cypress Hills, however, are unique in that they are also limited by a lack of suitable summer range. Since the study area is relatively depauperate of forested wetlands, increased pressure is put on resources which normally provide mostly winter browsing opportunities. As a result, such flora is extensively overbrowsed. In addition, moose are affected by a lack of dispersal opportunities in the area; no other suitable moose habitat exists anywhere near the Cypress Hills and Eagle Butte regions.

Mule deer and white-tailed deer are sympatric over much of their range in Alberta, however, the two species have differing habitat preferences. While white-tailed deer prefer interspersed forested habitats, mule deer frequent semi-arid, open forests and shrublands associated with steep, broken, or rough terrain. Both species do inhabit summer ranges 
within the Eagle Butte Sensitive Area, however the study area is critical to these species due to its provision of critical winter habitat (Table 3 ).

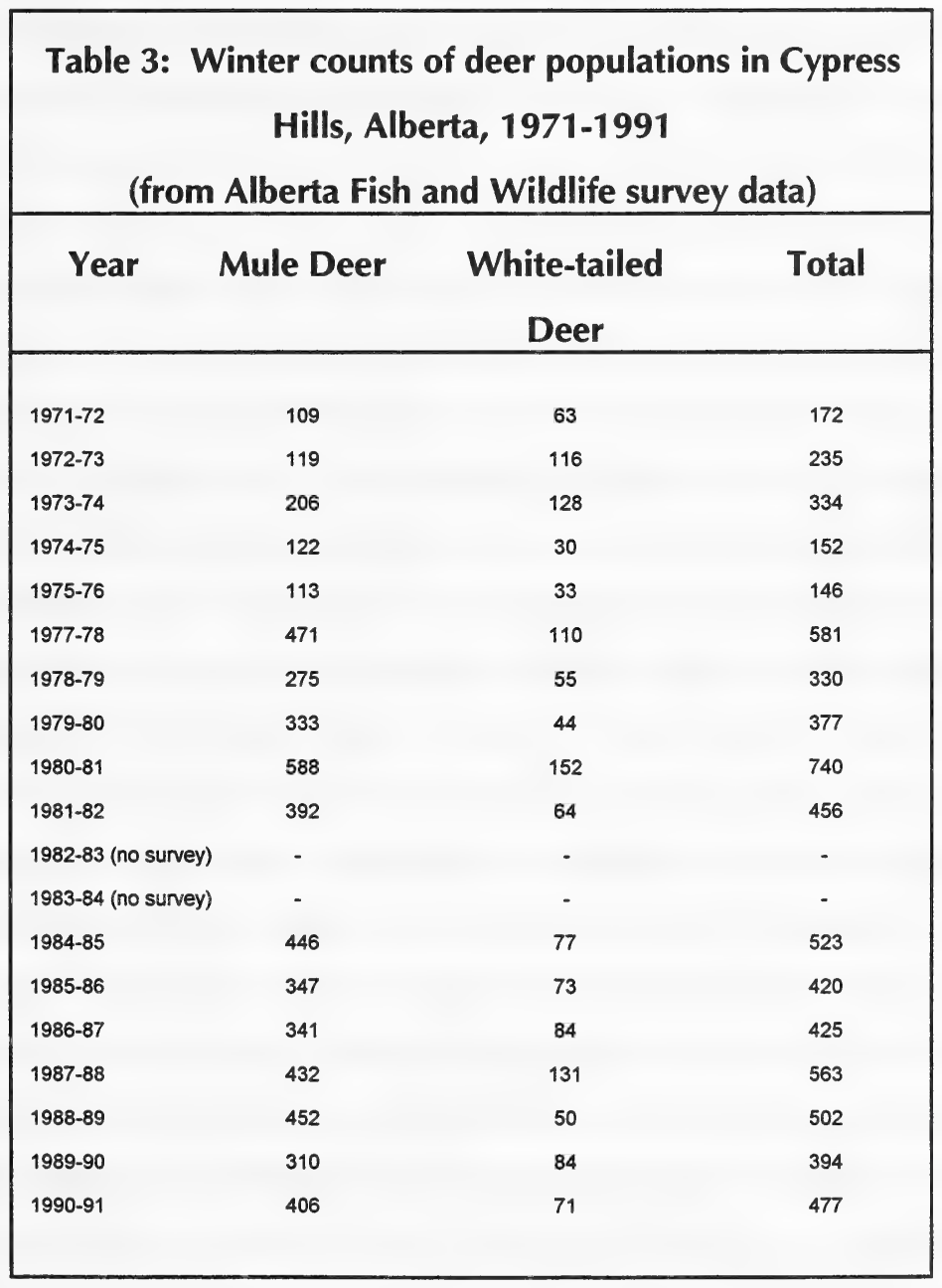

The insular nature of moose and, to a certain extent, elk populations in the Eagle Butte Sensitive Area are in stark contrast to populations of deer in the study area. While elk and 
moose have fairly stringent habitat requirements related to forage and cover resources, both species of deer venture onto open grassland habitats fairly regularly. Within the Cypress Hills and Eagle Butte areas, Lee (1979) found that deer made some use of all canopy cover types and vegetation associations with the exception of poplar wetlands. Lee's counts indicated a preference for mixedwood forests and for closed canopy coniferous stands with adjacent grasslands.

Migration of deer from summer ranges in the Cypress Hills to winter ranges in riparian shrublands and coulee bottoms is instigated by seasonal variations in weather conditions. Kramer (1971) recorded the movement of white-tailed deer out of the Cypress Hills and onto farmlands in winter in response to snow conditions and forage availability. Edwards (1974) reported that snow conditions, rather than snow depth, are largely responsible for high mule deer mortality during the winters in Cypress Hills Provincial Park. He cited high wind speeds and temperatures above freezing as factors which led to the formation of crusted or hardpacked areas, effectively impeding movement and feeding patterns. However, Kramer (op cit) found that while home ranges for mule deer and white-tailed deer overlap most during the spring, they overlap the least during the winter. Mule deer tend to overwinter within and near forest cover in both the Cypress Hills and Eagle Butte areas. Smith (1993), for example, reported mule deer winter concentrations on grassy slopes near woodlands on the summit of Eagle Butte. Deer populations at northern latitudes appear to be largely regulated by low temperatures and duration of snow cover rather than by range condition (Wishart 1986), thus emphasizing the importance of maintaining winter habitats such as are provided in the Eagle Butte Sensitive Area. 


\subsubsection{Other Mammals}

Apart from the ungulates, several other notable mammals occur, or potentially occur, in the Eagle Butte Sensitive Area. Westward expansion of human settlements in the Cypress Hills region, and the resultant land-use conflicts, are largely implicated in the loss of large carnivores from the region at the turn of the century. Presently, the smaller carnivores such as bobcat, red fox, coyote, and the mustelids are the only members of this group regularly inhabiting the Eagle Butte Sensitive Area, however, recent evidence of cougar has been cited by Alberta Fish and Wildlife Division (D. Eslinger, personal communication). As well, during field work conducted in 1995, notable mammal occurences were recorded in areas adjacent to the study area, within Cypress Hills Provincial Park, including black bear (Ursus americanus) sign (scat and tracks) as well as the confirmed sighting of a swift fox (Vulpes velox), both on the Cypress Hills plateau. In addition, numerous residents within the Eagle Butte Sensitive Area have confirmed sightings of, and attempted den establishment by, swift foxes in recent years.

Swift fox is designated provincially by the Alberta Wildlife Act as an Endangered Animal and nationally by COSEWIC as an extirpated species (AFWD 1991, COSEWIC 1995). The last confirmed Canadian sighting of this species was made in 1938 near Manyberries (Soper 1964) and the species has since been re-introduced to its native range on the Canadian prairies, with efforts initiated by the Canadian Wildlife Service in 1983. Currently, an estimated population of 200 swift foxes inhabit southeastern Alberta as a result of this reintroduction program but sufficient data is not yet available to accurately assess the reestablishment success of the population (L. Carbyn, personal communication).

Some researchers have correlated swift fox establishement with the sympatric coexistence of other species such as badger (Taxidea taxus) and ground squirrels (Herrero et al. 1991). 
Where locally abundant, it is anticipated that both species would provide numerous burrows which swift foxes would eventually occupy. Residents in the study area, however, cite attempted den establishment by swift foxes in association with numerous anthropogenic structures such as under cattle guards and inside drainage culverts.

Small mammal diversity is potentially high in the Eagle Butte Sensitive Area due to varying landforms and vegetation cover. One of the most common and most impacting small mammals in the study area is the northern pocket gopher (Thomomys talpoides), a relict member of the Great Basin Faunal Element, a group of species with affinities to sagebrush or rock outcrops. Northern pocket gophers reach their greatest densities on friable, coarsetextured soils which support good forage production (e.g., deep-rooted species such as Calamovilfa spp.). Portions of the grasslands in the western portions of the study area, characterized by soil textures ranging from light loam to fine sandy loam, and the riparian areas, characterized by Regosolic soils of varying textures, support pocket gophers in the study area and surrounding lands. Burrow systems developed by pocket gophers in some areas have resulted in severe soil disturbance, particularly on upland areas such as those surrounding Read Creek. Case (1983) reported as many as 200 soil mounds per gopher per year and up to 180 meters of tunnels in some habitats.

The small mammal community of the Eagle Butte Sensitive Area also consists of numerous other species, including disjunct populations of least chipmunk (Tamias minimus), southern red-backed vole (Clethrionomys gapperri), ermine (Mustela erminea), mink (Mustela vison), and snowshoe hare (Lepus americanus), all of which represent Boreal and/or Cordilleran Faunal Elements. The red squirrel (Tamiasciurus hudsonicus) was re-introduced to the Cypress Hills in a post-fire environment in 1886 and has become very abundant in pine- and spruce-dominated forests throughout the Cypress Hills and Eagle Butte areas. Still other species, such as white-tailed jack rabbit (Lepus townsendii), Richardson's ground squirrel 
(Spermophilus richardsonii), and thirteen-lined ground squirrel (Spermophilus tridecemlineatus) are representatives of a Campestrian Faunal Element, with ranges centered around semi-arid grassland environments of the Great Plains.

\subsubsection{Avifauna}

Numerous authors have estimated the number of bird species occuring in the Cypress Hills and Eagle Butte area at approximately 200 species, half of which breed in the region and half of which are seasonal migrants or winter residents (Halladay 1965, Wallis and Wershler 1980, Downs 1981, Biota Consultants Ltd. and Komex International 1992, Wallis 1992). Resident bird fauna of the Eagle Butte Sensitive Area is reflective of a complex habitat mosaic provided by the elevational gradients of the Cypress Hills and Eagle Butte. The higher portions of the Cypress Hills and Eagle Butte plateaus are in marked contrast to the semi-arid, rolling, treeless plains characteristic of much of the study area. The relatively cool and moist coniferous forests that occur atop Eagle Butte and Cypress Hills have been described by Wallis (1992) as "the only significant coniferous woodland habitats within the semi-arid mixed grassland area between south-central Manitoba and the Rocky Mountain foothills in Alberta."

The avifauna of the Eagle Butte Sensitive Area is characterized by an assemblage of species with affinities to both boreal, forested habitats as well as to semi-arid grassland habitats. This diverse bird community is able to persist in the Eagle Butte Sensitive Area due to the interspersion and gradation of habitats, ranging from forests to grasslands. The Native Grassland, Hummocky Grassland, and Native Grass/Shrub Complex vegetation communities described in Section 4.0 provide habitat for a variety of grassland birds such as savannah sparrow (Passerculus sandwichensis), vesper sparrow (Pooecetes gramineus), western meadowlark (Sturnella neglecta), horned lark (Eremophila alpestris), chestnut-collared 
longspur (Calcarius ornatus), McCown's longspur (Calcarius mccownii), lark bunting (Calamospiza melanocorys), sharp-tailed grouse (Tympanuchus phasianellus), and the endangered Baird's sparrow (Ammodramus bairdii).

The Baird's sparrow is a migratory bird which was designated as a "threatened" species by COSEWIC in 1989 due to population declines credited to habitat loss (DeSmet and Miller 1989). The species has recently been removed by COSEWIC (1996) from the Canadian Species At Risk list, indicating a level of recovery sufficient to warrant a delisting. There is currently a paucity of information on both historical and current population levels of the species, however, historical references found in Lane (1968) indicate that Baird's sparrows were abundant on the Great Plains prior to 1880 and that increased agricultural activity since the turn of the century have been a serious detriment to the species. Based solely on the rate of loss of grassland habitats, the overall population of Baird's sparrows is assumed to have been reduced by over 95 percent since historical times (DeSmet and Miller 1989). Recently, Wershler (1990) estimated a population of over 9,300 adult males in Alberta and this estimate has been used by AFWD (1991) to designate the species as "red-listed" in the province due to declining populations throughout its range, a trend which COSEWIC (1996) has indicated is reversing.

Preferred breeding habitat of Baird's sparrows is native grassland, particularly "extensive, idle, or lightly-grazed tracts of mixed grass prairie and local pockets of wet meadow zone or tallgrass prairie that are situated in lowland areas along the periphery of prairie ponds and lakes or along intermittent streams" (Stewart 1975). Within the Eagle Butte Sensitive Area, sites such as the Eagle Butte plateau, the hummocky moraine grasslands, and portions of the Cypress Hills provide typical nesting habitat for the Baird's sparrow in ungrazed or very lightly grazed fescue grassland dominated by rough fescue or mixed grassland dominated by spear grass. Wershler (1990) reported Baird's sparrow occurrence from numerous 
microhabitats in southern Alberta, including native fescue, mixed grasslands, dry grassy basins, meadow grasses surrounding small drainages, and exotic habitats such as cropland and various crested wheat-brome-alfalfa combinations. Both cattle grazing and the breaking and reseeding of native pastures for cultivar production have contributed to the loss of available habitat for Baird's sparrows. There is strong evidence, presented by Dale (1983), that grazed grasslands support considerably less Baird's sparrows compared with ungrazed areas, despite the fact that grazing itself has resulted in the continued existence of grasslands. Typical grazing practices are not conducive to the maintenance of Baird's sparrow habitat, which includes lush grassland with a well-developed understory. Cropland also is not a suitable replacement for native grassland (DeSmet and Conrad 1989).

Haying of native grassland and tame forage is a widespread agricultural practice in much of the grassland areas of the Eagle Butte Sensitive Area. The cutting of tame forage occurs mainly in June and July, coinciding with the peak nesting period for many bird species (Dale 1993). However, mowing of native cover usually occurs later in the summer, thus allowing birds to raise broods prior to cover removal. Hayfields and tame pastures are generally not as productive as natural habitats for most grassland bird species, including the Baird's sparrow. Since alteration of key prairie habitats is cited by most authors as the primary threat to Baird's sparrow throughout its Canadian range, the maintenance of natural grassland refugia such as those present in the Eagle Butte Sensitive Area is a crucial step towards the recovery and conservation of Baird's sparrows. From a broader conservation perspective, Baird's sparrows also act as an umbrella species, because providing for the needs of this species also postively affects other species such as grasshopper sparrow and Sprague's pipit, which are closely associated with Baird's sparrow in some habitats.

Sharp-tailed grouse are a widely distributed species in the province, inhabiting suitable habitats in numerous Natural Regions of Alberta. It is a fairly common species in the 
Grassland, Central Parkland, and Peace River Parkland regions. In the Eagle Butte Sensitive Area, sharp-tailed grouse have been recorded to inhabit open prairie, coulee, and water course margin habitats. Habitat mosaics of shrubs, grasses, and trees, such as are found in the Riparian Shrubland Draw, Native Grass/Shrub Complex, and Mixed Wood Forested community types in the Eagle Butte Sensitive Area, yield the highest densities of sharp-tailed grouse. The establishment of territories on and surrounding leks is a primary requirement of maintaining populations of sharp-tailed grouse. Territorial males require slightly elevated, open, flat areas which afford the displaying grouse a wide field of vision in all directions. Nest sites of brooding females are generally located in very close proximity to dancing grounds. Nietfeld et al. (1984) reported that nest sites are usually within $1 \mathrm{~km}$ of the dancing grounds, while Alberta Fish and Wildlife Division has confirmed that a zone of $2 \mathrm{~km}$ surrounding leks will be recognized by the current ESSA program as a critical area (D. Eslinger, personal communication; J. Taggart, personal communication). Therefore, conserving adequate habitat for dancing grounds is imperative for continued viability of the species and has been acknowledged as such.

Of the forested communities that occur in the Eagle Butte Sensitive Area, the Forested Mixed Wood community type offers the most diverse habitat for breeding forest birds. Aspen- or conifer-dominated mixedwood forests are characterized by a high degree of vertical heterogeneity, a result of interspersed canopy gaps which allow for the development of prominent shrub, forb, and graminoid understories. This vertical and horizontal heterogeneity, as defined by Hunter (1990), provides differentiated and stratified niches and a relatively large accumulation of biomass which, in turn, supports a diverse food web.

Physiographic variation within the Eagle Butte Sensitive Area - provided by the interspersion of open grassland, mixedwood forests, deciduous forests, and coniferous forests - results in its avian inhabitants displaying considerable variation in migratory patterns (permanent 
residents, short-distance migrants, and long-distance migrants); foraging guilds (granivores, insectivores, and carnivores); and nest site selection (cavity nesters, ground nesters, and brood parasites). Wallis (1992) identifed 20 species of forest-dwelling birds of particular interest inhabiting the Cypress Hills area (Table 4).

\section{Table 4: Significant species of forest-dwelling birds in the Cypress Hills} (adapted from Wallis 1992)

$\begin{aligned} \text { Cooper's hawk } & \text { Accipiter cooperii } \\ \text { northern goshawk } & \text { Accipiter gentilis } \\ \text { sharp-shinned hawk } & \text { Accipiter striatus } \\ \text { broad-winged hawk } & \text { Buteo platypterus } \\ \text { saw-whet owl } & \text { Aegolius acadicus } \\ \text { common poor-will } & \text { Phalaenoptilus nuttalli } \\ \text { red-headed } & \text { Melanerpes erythrocephalus } \\ \text { woodpecker } & \\ \text { red-naped sapsucker } & \text { Sphyrapicus nuchalis } \\ \text { three-toed } & \text { Picoides tridactylus } \\ \text { woodpecker } & \\ \text { pileated woodpecker } & \text { Dryocopus pileatus }\end{aligned}$

$\begin{aligned} \text { Swainson's thrush } & \text { Catharus ustulata } \\ \text { red-breasted nuthatch } & \text { Sitta canadensis } \\ \text { brown creeper } & \text { Certhia familiaris } \\ \text { golden-crowned kinglet } & \text { Regulus satrapa } \\ \text { solitary vireo } & \text { Vireo solitarius } \\ \text { ovenbird } & \text { Seiurus aurocapillus } \\ \text { western tanager } & \text { Piranga ludoviciana } \\ \text { red crossbill } & \text { Loxia curvirostra } \\ \text { white-winged crossbill } & \text { Loxia leucoptera } \\ & \\ \text { Cassin's finch } & \text { Carpodacus cassinii }\end{aligned}$

Of these species cited by Wallis (op cit), only Cooper's hawk is designated by COSEWIC as being a vulnerable species (COSEWIC 1995), while the common poor-will and the redheaded woodpecker are both rare species in Alberta. Salt and Salt (1976) considered the poor-will to breed in the Cypress Hills area, probably the only breeding locale in Alberta, where the species is at the western periphery of its range. Semi-arid sagebrush benchlands and grassy openings of dry, open woodlands provide appropriate breeding habitat in the Cypress Hills and Eagle Butte regions (Semenchuk 1992). 
Turkey vultures (Cathartes aura) are a fairly common species in the Eagle Butte Sensitive Area as well. Numerous individuals were recorded during field work in the summer of 1995. Semenchuk (1992) considered the species to be uncommon in Alberta, where it is at the northern limit of its range. AFWD (1991) reports that the Alberta population of turkey vultures is likely less than 100 pairs and that summer breeding records were concentrated in the Cold Lake and Big Valley areas of the province. While they do not generally nest in trees, turkey vultures' requirements for roosting trees near stable food and water sources necessitates groveland habitats with adjacent open foraging areas. Nesting habitats, which include rocky outcrops and protected caves and crevices in cliffs, are generally limited in the Eagle Butte Sensitive Area, and those that can be identified should be protected from disturbance.

The Cypress Hills and Eagle Butte areas are home to Canada's only population of the pinksided form of dark-eyed junco (Junco hyemalis mearnsi). This race of sparrow is one of four races of juncos that can be found in Alberta, but Mearn's, or pink-sided, race is known only from the Cypress Hills, where it is considered fairly common in mixedwood forest edges.

Waterfowl use is extremely limited in the Eagle Butte Sensitive Area, due to the lack of permanent wetlands throughout most of the study area. Wetland formation and permanence is most pronounced in the hummocky moraine landscape which characterizes the Hummocky Grassland community type in the northern portion of the study area. Both diving and dabbling ducks as well as numerous species of shorebirds and waterbirds have been recorded in this portion of the Eagle Butte Sensitive Area. Extensive fresh-water springs and perched wetlands along the slopes of the Cypress Hills also provide habitat for waterbirds. However, the fluctuating nature of water levels in these wetlands as a result of extensive spring drawdown results in these habitats providing seasonal staging habitats rather than summer nesting habitats. Nesting concentrations of waterbirds are found mostly in the 
northern hummocky moraine, in Michel Reservoir, and in the small portion of Elkwater Lake which extrudes into the study area.

Semenchuk (1992) indicates that at least nine species of waterfowl have been recorded as breeding in the Cypress Hills area, including mallard (Anas platyrhynchos), blue-winged teal (Anas discors), gadwall (Anas strepera), American wigeon (Anas americana), ring-necked duck (Aythya collaris), lesser scaup (Aythya affinis), white-winged scoter (Melanitta fusca), bufflehead (Bucephalus albeola), and ruddy duck (Oxyura jamaicensis). While not all of the above species were verified during field work in the summer of 1995, it is anticipated that many of the diving ducks would be limited largely to the deeper, more permanent waterbodies such as Elkwater Lake and Michel Reservoir. Michel Reservoir was also found to be inhabited by numerous red-necked grebes (Podiceps grisegena), horned grebes (Podiceps auritus) and Canada geese (Branta canadensis).

\subsubsection{Herptiles}

Amphibians are often abundant and functionally critical elements in most terrestrial and many freshwater ecosystems and, therefore, constitute a significant component of the world's biological diversity. However, Bishop and Pettit (1992) and numerous other researchers recognize that there is currently a dearth of knowledge about the health and stability of most populations of amphibians and reptiles worldwide. Despite this paucity, most authors also acknowledge recent global declines in amphibian populations (Barinaga 1990, Blaustein and Wake 1990). As a group, herptiles are also acknowledged as the most endangered group of organisms in prairie Canada (WWFC 1988, Quinn 1991). Causes for declines, both globally and in North America, that have been proposed by D. Seburn (1993) include acid precipitation, airborne pesticides, and ultraviolet light. Clearly, habitat degradation should 
be added to this list, as losses caused by livestock grazing and wetland infilling are major causative factors of declining prairie biodiversity.

The herpetofaunal assemblage of Alberta is generally not regarded as being extensive, with the greatest concentration of species occuring in the arid southeastern corner of the province. A total of eight species of reptile and 10 species of amphibian are known to occur in Alberta but ranges described by Russell and Bauer (1993) indicate that only seven species - tiger salamander (Ambystoma tigrinum), striped chorus frog (Pseudacris triseriata), wood frog (Rana sylvatica), northern leopard frog (Rana pipiens), painted turtle (Chrysemys picta), wandering garter snake (Thamnophis elegans), and plains garter snake (Thamnophis radix) - potentially occur in the study area. Additional species are present in the more arid regions surrounding the elevated Cypress Hills.

Most of the herpetofaunal species present in the study area occur in abundance and their population trends are relatively stable. The notable exception, however, in the study area is the northern leopard frog, a species which has witnessed a considerable decline since the mid-1960s in the U.S. portions of its range and since the late-1970s in Alberta. The northern leopard frog was once the most widespread frog species in North America and was abundant throughout eastern, central and southern Alberta. Roberts (1981) noted the total disappearance of the northern leopard frog from much of its range in 1979 - from sites where the species had not been only present, but abundant, a year earlier. As of 1990, only 32 locations of northern leopard frog were known from Alberta and only 17 of these populations showed evidence of breeding (AFWD 1990).

It is generally accepted that central Alberta populations of northern leopard frog have been extirpated (Roberts 1992) and that all extant populations of the species now occur at or south of the Red Deer River and in the extreme northeastern corner of the province. In the Eagle 
Butte Sensitive Area, the edges of streams and small wetlands and beaver ponds in the study area provide potential habitat for the species and sites such as Sexton Creek in the Eagles Nest Candidate Natural Area have been recognized as providing the most important leopard frog habitat in the province (Biota Consultants Ltd. and Komex International 1992). During field work in the summer of 1995, numerous leopard frogs were observed in three wetland complexes along Sexton Creek and along Lodge Creek and a tributary drainage.

The western painted turtle is a local and very rare species, occurring in Alberta at the very northern limit of its range. There are several records of this species from oxbow lakes and slow moving streams in the Cypress Hills (Wallis 1992). Biota Consultants Ltd. and Komex International (1992) also report the observation of two painted turtles from the Sexton Creek area, however it is not known whether these individuals are indigenous or introduced. Naturally occuring populations of western painted turtles have been recorded from the lower reaches of the Milk River, a northern extension of the Missouri River drainage system. Wallis (1986) and Biota Consultants Ltd. and Komex International (1992) also reported the last local sighting of painted turtles to have been made in the Battle Creek area of Cypress Hills Provincial Park in 1979 and both authors indicate that these populations may be naturally occuring, although there is no data to substantiate such statements.

\subsubsection{Significant Fauna By Community Type}

Biophysical factors considered to directly influence habitat suitability can include vegetation cover type, slope, aspect, and other topographic features. Since vegetation cover type is actually an expression of a variety of biophysical conditions such as soil, aspect, and relief, it generally offers the most current and valid prediction of habitat suitability for most wildlife species. Vegetation composition and structure indicate, both directly and indirectly, the quality of food and cover available for many faunal groups and species. Furthermore, 
vegetation cover is dynamic, being subject to change as a result of fire, flooding, grazing, haying, and/or natural succession. Ultimately, it is the balance between flora and fauna that is responsible for the evolution of behavioral and reproductive patterns in wildlife.

For many wildlife species of management or conservation concern, a determination of the current condition and trend of the available habitat provides a reliable means of monitoring associated wildlife species. With this in mind, the re-interpretation of the previously identified "community types" as "habitat types" will allow for a correlation between vegetation cover and wildlife use. Fourteen vegetation community types have been previously identified in Section 4.0. From the perspective of wildlife habitat, these community types can be described by five broadly delineated habitat types:

1. Native Grassland;

2. Hummocky Grassland;

3. Riparian;

4. Forest; and

5. Wetland

\section{Native Grassland Habitats}

Native grassland habitats in the Eagle Butte Sensitive Area include the previously defined Native Grassland, Native Grass/Shrub Complex, Coulee Slopes, and Eroding Slopes community types. The variation in native grassland habitats in the Eagle Butte Sensitive Area 
is a result, primarily, of the aspect and elevation of the site and, secondarily, of the composition of the vegetation. Fescue grassland occurs on plateau areas and on dry, exposed slopes in the study area. Smith (1993) reported fescue grassland with a significant component of grass cover to be quite restricted in the Eagle Butte area, occuring only on limited plateau areas and on undulating slopes. These lush grasslands with limited shrub cover are preferred habitat for bird species such as Baird's sparrow, Sprague's pipit, clay-colored sparrow, and vesper sparrow. Baird's sparrow, western meadowlark, and savannah sparrow are intolerant of heavily grazed grasslands, however vesper sparrows and Sprague's pipit are found in lightly to moderately grazed grasslands.

Species commonly associated with native grassland habitats in the Eagle Butte Sensitive Area are northern pocket gopher, badger, and white-tailed jack rabbit. Given an abundant small mammal prey base, these fescue grasslands occuring adjacent to forest cover are also preferred foraging areas for predators such as red-tailed hawk, northern harrier, merlin, and kestrel. Elk and mule deer also preferentially feed in fescue grasslands, particularly where adjacent forest cover is present.

Low shrubs such as roses, shrubby cinquefoil, and, more commonly, buckbrush, provide scattered local areas of cover and nesting sites for endemic grassland birds, small mammals, and even ungulates.

\section{Hummocky Grassland Habitats}

Hummocky grassland habitats in the Eagle Butte Sensitive Area are represented by a single previously identified community type - the Hummocky Grassland. This community type, located predominantly in the hummocky moraine area on the north slopes of the Cypress Hills, contains interspersed fescue grassland on upland areas between pockets of small ponds 
which occupy the depressions. This knob-and-kettle topography provides habitat which is closely associated with that of the Riparian community type.

The shrub thickets bordering and interspersed amongst the wetlands provide valuable forage and cover for deer. Numerous sharp-tailed grouse dancing grounds have also been identified in upland areas of such hummocky grasslands.

\section{Riparian Habitats}

Riparian habitats in the Eagle Butte Sensitive Area include habitats from the previously defined Alluvial Fans/Floodplains, Riparian, and Shrub Draws community types. The common link between all of these community types is that they are characterized and defined by the action of water and drainage activity.

The importance of these habitats for wildlife stems from their highly productive nature which allows for the growth of tall shrubbery and, in some cases, the only woody vegetation that is found in large areas otherwise devoid of woody vegetation. Tall shrub growth, including varying mixtures of hawthorn, willow, saskatoon, and choke cherry, house a diverse breeding bird community and provide dispersal and movement corridors as well as cover and forage for ungulates such as elk, deer, and moose. Winter ranges of elk in Cypress Hills Provincial Park have been shown to closely follow the distribution of upland shrubs, particularly hawthorn and silverberry (Ecoplans Limited 1982).

Additionally, bird species such as MacGillivray's warbler (Oporornis tolmei), common yellowthroat (Geothlypis trichas), American redstart (Setophaga ruticilla), Brewer's blackbird (Euphagus cyanocephalus), cedar waxwing (Bombycilla cedrorum), and the American goldfinch (Spinus tristis) all select tall shrubbery habitats in wetland and/or riparian margins. 


\section{Forested Habitats}

Forested habitats in the Eagle Butte Sensitive Area are comprised of the previously defined Deciduous Forest, Coniferous Forest, and Mixed Wood Forest community types. These forested communities are largely concentrated at higher elevations on the northern and western slopes of the Cypress Hills. The gradation between each of these forested habitats is often gradual, resulting in numerous ecotonal zones which are of high value to wildlife.

Coniferous forests in the Eagle Butte Sensitive Area are dominated by pure stands of lodgepole pine, however, some pure stands of white spruce also occur. Both pine and spruce stands produce very little palatable browse or forage material and, therefore, wildlife use of these stands is restricted to the provision of shelter and cover. Shallow winter snow depths in pine stands, attributed to a dense and closed canopy, results in high winter use by ungulates in the area. Immature pine stands are also inhabited by snowshoe hare and bobcat, while older stands provide habitat for species such as red squirrel. Ecoplans Limited (1982) list three bird species - Swainson's thrush, golden-crowned kinglet, and ruby-crowned kinglet - which use spruce forests exclusively. Use of conifer-dominated forests by larger mammals, particularly ungulates, however is seasonal and is most often noted in ecotonal zones.

Deciduous forests in the Eagle Butte Sensitive Area are dominated by aspen and, sometimes, balsam poplar. Such stands have been shown to provide habitat for songbirds, raptors, ungulates, and small mammals. Examples of bird species which rely on heavy deciduous woodlands include ruffed grouse, northern saw-whet owl, red-eyed vireo (Vireo olivaceus), orange-crowned warbler (Vermivora celata), ovenbird, downy woodpecker (Picoides pubescens), and mountain bluebird (Sialia currocoides). Aspen suckers have also been shown to be a preferred browse item of all of the region's ungulates - moose, elk, mule deer, 
and white-tailed deer. Relict populations of snowshoe hare and porcupine are also notable species in deciduous woodlands of Eagle Butte Sensitive Area while microtines are usually more abundant in deciduous stands than in other forested stands because of the high degree of winter insulation associated with heavy snowpacks.

Mixedwood forests are transitional in nature and are found in the middle position of elevational and moisture gradients. Therefore, these forest stands are found on the most types of soils and on a wide variety of aspects and moisture regimes and is the most extensive forest community type in the Eagle Butte Sensitive Area. Mixedwood habitats are the most productive habitats in the Eagle Butte Sensitive Area for wildlife because they provide a great variety of microhabitats and vertically differentiated niches. Moose, white-tailed deer, and mule deer all make extensive use of deciduous saplings and shrubs while elk also make use of these habitats in the face of increasing competition from cattle for grazing resources.

Small mammals such as southern red-backed vole, deer mouse (Peromyscus maniculatus), and long-tailed vole (Microtus longicaudus) as well as red squirrel and least chipmunk (Eutamias minimus) are very common in mixedwood habitats. Seven species of raptors and one owl, including great gray owl (Bubo virginianus), broad-winged hawk, northern goshawk, Cooper's hawk, sharp-shinned hawk, red-tailed hawk, Swainson's hawk, and merlin (Falco columbarius), nest in mixedwood forests and hunt in adjacent forest openings and clearings.

\section{Wetland Habitats}

Wetland habitats are found in the previously defined Permanent Wetland and Ephemeral Wetland community types. Some of these wetlands, particularly those that are permanent, provide habitat for a variety of shorebirds, waterfowl, and other waterbirds and contain some of the very few areas of standing water in the study area. Northern leopard frog and western 
painted turtle occurences have been reported from many of these wetlands as well. Large, permanent wetlands such as Michel Reservoir and Elkwater Lake provide habitat for waterbirds, while permanent wetlands located in wooded habitats are inhabited by beaver (Castor canadensis) and modified by them to the advantage of species such as northern leopard frogs.

All herptiles which occur in the Eagle Butte Sensitive Area are restricted to wetland habitats for all or a portion of their lives. Striped chorus frog, northern leopard frog, wood frog, western painted turtle, tiger salamander, and plains garter snake all have strong affinties to wetland habitats and the wandering garter snake is also most common near, but not restricted to, wetland habitats.

Table 5 on the following page provides a summary of selected significant fauna and their expected and observed occurrence within the previously defined vegetation community types of the Eagle Butte Sensitive Area. 
Biophysical OVerView, Significant, Sensitive and Disturbance

Table 5: Significant Fauna and Habitat Use By Vegetation Community Type

\begin{tabular}{|c|c|c|c|c|c|c|c|c|c|}
\hline & moose & elk & $\begin{array}{l}\text { swift } \\
\text { fox }\end{array}$ & $\begin{array}{l}\text { sharp- } \\
\text { tailed } \\
\text { grouse }\end{array}$ & $\begin{array}{c}\text { dusky } \\
\text { flycatcher }\end{array}$ & $\begin{array}{c}\text { Mearn's } \\
\text { dark- } \\
\text { eyed } \\
\text { junco } \\
\end{array}$ & $\begin{array}{l}\text { Baird's } \\
\text { sparrow }\end{array}$ & $\begin{array}{l}\text { mountain } \\
\text { bluebird }\end{array}$ & $\begin{array}{c}\text { northern } \\
\text { leopard } \\
\text { frog }\end{array}$ \\
\hline Native Grassland & & & $\bullet$ & $\bullet$ & & & $\bullet \bullet \bullet$ & & \\
\hline $\begin{array}{l}\text { Native Grass / } \\
\text { Shrub Complex }\end{array}$ & & $\bullet$ & & $\bullet \bullet \bullet$ & & $\bullet$ & - & & \\
\hline Coulee Slopes & & - & $\bullet$ & $\bullet$ & & & & & \\
\hline Eroding Slopes & & $\bullet$ & & $\bullet$ & & - & & & \\
\hline $\begin{array}{l}\text { Alluvial Fans } \\
\text { /Floodplains }\end{array}$ & & $\bullet$ & & $\bullet$ & • & & & & \\
\hline $\begin{array}{l}\text { Hummocky } \\
\text { Grassland }\end{array}$ & & - & & $\bullet$ & & - & $\bullet$ & & \\
\hline $\begin{array}{l}\text { Permanent } \\
\text { Wetland }\end{array}$ & & & & & & & & & $\bullet \bullet$ \\
\hline $\begin{array}{l}\text { Ephemeral } \\
\text { Wetland }\end{array}$ & $\bullet$ & $\bullet$ & & $\bullet$ & & & & & $\bullet$ \\
\hline Riparian & $\bullet$ & $\bullet$ & & & $\bullet$ & & & & $\bullet$ \\
\hline Shrub Draws & - & $\bullet$ & & $\bullet$ & $\bullet$ & & & $\bullet$ & \\
\hline Deciduous Forest & $\bullet$ & $\bullet \bullet \bullet$ & & & $\bullet \bullet$ & & & $\bullet \bullet$ & \\
\hline Coniferous Forest & $\bullet$ & $\bullet$ & & & & $\bullet$ & & & \\
\hline $\begin{array}{l}\text { Mixed Wood } \\
\text { Forest }\end{array}$ & $\bullet$ & $\bullet$ & & - & $\bullet$ & $\bullet \bullet \bullet$ & & $\bullet \bullet$ & \\
\hline $\begin{array}{l}(\bullet) \text { - indicate } \\
(\bullet \bullet) \text { - indicat }\end{array}$ & $\begin{array}{l}\text { ome } \\
\text { mod }\end{array}$ & $\begin{array}{l}\text { e } \\
\text { ate } u\end{array}$ & & & & & & & \\
\hline
\end{tabular}




\subsection{Significant Areas}

The following is a summary of significant areas identified in the Eagle Butte Sensitive Area which are mappable, as presented in Map 3. Key features of each site are also listed. A detailed discussion of each site can be found in Section 9.0.

Nationally Significant Areas

\begin{tabular}{|c|c|}
\hline Area Name & Key Features \\
\hline $\begin{array}{l}\text { Cypress Hills } \\
\text { (page 124) }\end{array}$ & $\begin{array}{ll}\text { - } & \text { part of one of the few large blocks of native prairie } \\
\text { remaining in Canada } \\
\text { - } & \text { unglaciated and glaciated landscape } \\
\text { - } & \text { geologically diverse } \\
\text { - } & \text { numerous fresh water springs } \\
\text { - } & \text { relict rough fescue grasslands } \\
\text { - } & \text { forests with western Cordilleran affinities } \\
- & \text { numerous rare, restricted and disjunct plant species } \\
- & \text { habitat for burrowing owl and swift fox } \\
- & \text { critical winter habitat for elk, mule deer, white- } \\
\text { tailed deer and moose } \\
\text { sharp-tailed grouse leks } \\
\text { - } \quad \text { hibernacula for red-sided garter snakes } \\
\text { provincially important habitat for northern leopard } \\
\text { frog }\end{array}$ \\
\hline $\begin{array}{l}\text { Eagle Butte Uplands } \\
\text { (page 127) }\end{array}$ & 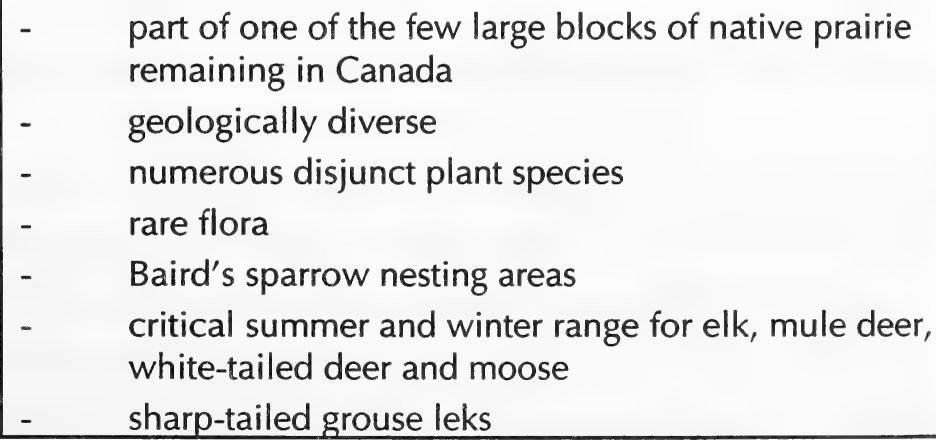 \\
\hline
\end{tabular}




\begin{tabular}{||l|l||}
\hline $\begin{array}{l}\text { Hummocky Moraine } \\
\text { (page 129) }\end{array}$ & $\begin{array}{l}\text { part of one of the few large blocks of native prairie } \\
\text { remaining in Canada }\end{array}$ \\
& $\begin{array}{l}\text { Baird's sparrow nesting areas } \\
\text { numerous ephemeral and permanent wetlands } \\
\text { providing significant waterfowl and shorebird } \\
\text { staging habitats } \\
\text { sharp-tailed grouse leks }\end{array}$ \\
\hline
\end{tabular}

\section{Provincially Significant Areas}

\begin{tabular}{||l|ll||}
\hline \multicolumn{1}{|c||}{ Area Name } & \multicolumn{1}{c||}{ Key Features } \\
\hline \hline $\begin{array}{l}\text { Medicine Lodge Coulee } \\
\text { - Lodge Creek } \\
\text { (page 131) }\end{array}$ & - & $\begin{array}{l}\text { northern leopard frogs } \\
\text { critical summer and winter ranges for mule deer } \\
\text { sharp-tailed grouse leks } \\
\text { pronghorn antelope habitat in extreme southern } \\
\end{array}$ \\
& - & $\begin{array}{l}\text { portion } \\
\text { part of Missouri drainage system }\end{array}$ \\
& - & \\
\hline
\end{tabular}

\section{Regionally Significant Areas}

\begin{tabular}{|c|c|}
\hline Area Name & Key Features \\
\hline $\begin{array}{l}\text { Medicine Lodge Coulee } \\
\text { - Bullshead Creek } \\
\text { (page 133) }\end{array}$ & $\begin{array}{ll}\text { - } & \text { rare flora } \\
\text { - } \quad \text { headwaters of Bullshead Creek } \\
\text { - } \quad \text { extensive disturbance from agricultural development }\end{array}$ \\
\hline
\end{tabular}

Locally Significant Areas

\begin{tabular}{|c|c|}
\hline Area Name & Key Features \\
\hline $\begin{array}{l}\text { Manyberries Creek } \\
\text { (page 135) }\end{array}$ & $\begin{array}{l}\text { - } \quad \text { headwaters of Manyberries Creek } \\
\text { - } \quad \text { extensive disturbance from agricultural development }\end{array}$ \\
\hline
\end{tabular}




\subsection{SENSITIVE FEATURES}

Environmental sensitivity ratings are often used as an evaluation of the performance of a site in response to various land uses or disturbance types (Bentz and Saxena 1993, O'Leary et al. 1993). On lands which have a low sensitivity to disturbance, many disturbances can be easily remedied by standard operating procedures. Conversely, other lands may be more sensitive to disturbance because they possess at least one of the following characteristics (Bentz and Saxena 1993):
- a very high susceptibility to erosion
- severe limitations to revegetation, or
- distinctive, rare or unusual landforms, wildlife populations or plant communities that are regionally, provincially, nationally or internationally important.

The Eagle Butte Sensitive Area possesses all of the above characteristics, to varying degrees. Southeastern Alberta as a region is generally an area of high wind erosion risk (Coote and Pettapiece 1989). The Eagle Butte Sensitive Area itself is located in an area which, on a broad scale, is considered as having moderate to high wind erosion risk, with loam and sandy loam surface soil textures and high wind speeds predominant.

Much of the study area is also within a severe water erosion risk area (Tajek et al. 1985), and the effects of water erosion are prominent in the study area as evidenced by the extensive dissected topography, the Eroding Slopes community type and extensive fan areas within the Alluvial Fan/Floodplain community type. 
The Eagle Butte Sensitive Area can therefore be considered as a physically fragile area in general. However, some areas within the study area can be considered more fragile than others, and this difference is presented in Map 4 as a rating of the sensitivity of sites to physical disturbance, defined here as the degree of anticipated negative effects, usually as caused by wind or water erosion, if the ground cover is disturbed such as by petroleum exploration activities.

Highly sensitive sites are those where disturbance would result in a severe loss of environmental significance or value, or would require the use of major mitigative techniques and very restrictive operating conditions to minimize this loss. Site reclamation would be expected to be very difficult, and the potential for the site to be successfully reclaimed would be anticipated to be low.

Sites with a moderate sensitivity rating could suffer a significant reduction in environmental significance as a result of disturbance, and would require restrictive operating conditions and specialized mitigation and reclamation techniques to maintain the values of the site.

Sites with a low sensitivity are anticipated to experience insignificant negative effects resulting from physical disturbance within normal operating conditions.

Sensitivity ratings for the study area were generally based on slope class and slope type, though other edaphic and biotic factors, as described by Bentz and Saxena (1993) and outlined above, were also considered to derive the ratings.

A significant portion of the Eagle Butte Sensitive Area has been classed as having very high or high sensitivity to disturbance. Areas with a very high sensitivity to disturbance include 
areas of very strong relief, usually the result of erosion by water or by extensive slumping. Community types often corresponding to these areas include the Eroding Slopes, Shrub Draw and forested community types. These are areas of complex slopes with strong relief. Erosional effects subsequent to physical disturbance are expected to be severe, and reclamation success is anticipated to be low. Numerous springs are located in these areas, and which provide potab; e water and support rare plant populations in many cases. The Eroding Slopes community type may also provide habitat for rare flora and fauna.

The Riparian, Permanent Wetland, Ephemeral Wetland, Coulee Slopes, Native Grass/Shrub Complex and portions of the Native Grassland, Alluvial Fans/Floodplain, Hummocky Grassland and forested community types are rated as highly sensitive to disturbance, due to either their physical or biological attributes or both. For example, significant portions of the Hummocky Grassland community type are classed as being highy sensitive to disturbance to very "hummocky" topography with strong, short slopes. Any removal or disturbance to the ground cover in this area could result in accelerated surface erosion. Depressional areas within this topography are poorly drained. Ephemeral wetlands are poorly drained and very easily disturbed by motor vehicles, as are low-lying saline areas in extreme southern portions of the study area, which are poorly vegetated and poorly drained. As well, wetlands within the study area support populations of northern leopard frogs, a "Red-listed" species in Alberta with a declining population. One wetland is also known to support a small population of western painted turtles. Specific wetlands which support northern leopard frog or western painted turtle populations should be considered as very highly sensitive to disturbance. Thus, site specific investigations of permanent and ephemeral wetlands would be prudent prior to any development activities in the vicinity of these areas.

Portions of the Native Grassland, Native Grass/Shrub Complex, Hummocky Grassland and Alluvial Fan/Floodplain community types are rated as having moderate sensitivity to 
disturbance. Topography in these types is relatively subdued. Some of these community types, particularly the Alluvial Fan/Floodplain and depressional areas within the Hummocky Grassland community type, reflect the effects of high water tables or poor drainage to varying degrees. However, subsequent to physical disturbance, areas with a moderate sensitivity rating are expected to have a high reclamation success with the employment of reclamation techniques adapted to regional climatic, soil and vegetation conditions.

Areas classed as having a low sensitivity to disturbance include portions of the Native Grassland community type on well drained, gentle slopes such as found south of Cypress Hills Provincial Park. Significant portions of the Alluvial Fan/Floodplain community type within Medicine Lodge Coulee are also classed as having a low sensitivity to disturbance for similar reasons.

Discussions of sensitivity ratings within the study area are best considered in relation to the prevailing land and resource management philosophies of the time. For example, during the 1970 's to early 1980 's, wellsites were often located within portions of some sensitive areas, such as within the Manyberries Badlands Sensitive Area, that are rated as having very high to high sensitivity to disturbance. The rationale of the time was that poorly vegetated, actively eroding areas under normal, "natural" conditions would be suitable wellsite locations, since the areas were already actively eroding anyway, and it would keep disturbances away from well-vegetated grassland areas (J. Clark, pers. comm., T. Hood, pers. comm.). In contrast, current management philosophies are quite different. Land and resource managers now encourage petroleum activities to be directed away from eroding areas and on to well-vegetated grassland sites, in order to discourage the acceleration of erosional processes, to maintain aesthetic values, to protect important wildlife species, and to ensure better reclamation success by locating these disturbances on productive, vegetated sites (J. Clark, pers. comm.). 
It should also be noted that sensitivity ratings as presented on Map 4 should only serve as a general indication of sensitivity within each polygon. Site-specific investigations are recommended prior to development activity at any particular site. 


\subsection{DISTURBANCE FEATURES}

The impact of man's activities on the Eagle Butte Sensitive Area is readily observed from both the ground and from aerial photographs. Large scale disturbances have been caused by the active cultivation of land and the creation of tame or "improved" pasture. Linear disturbances have resulted from the development of petroleum and range resources within the area. Disturbance features are depicted on Map 5.

It appears that land totalling nearly 61 quarter sections has previously been broken within the Eagle Butte Sensitive Area for agricultural purposes. This equates to approximately 12 percent of the entire study area. Cultivation for the most part has been limited to lands with simple slopes of low relief, as found on the Cypress Hills Plateau in the southeastern portion of the study area, within the valley bottoms of Medicine Lodge Coulee and Manyberries Creek valley, and on localized relatively level areas scattered through the remainder of the study area. Cultivated units shown on Map 5 represent lands currently under cultivation, improved pasture areas, and areas which have been cultivated in the past but which are now reverting to native prairie.

Associated with this agricultural development are 20 permanent farm residences, trails, drainage ditches, 38 dugouts, 43 reservoirs and 11 dry dams'. While none of these disturbances are significant by themselves, combined they result in a relatively extensive disturbance pattern throughout the area. For the most part, the farm residences, trails and drainage ditches are in the vicinity of the cultivated fields.

1. Fencelines were not mapped due to time constraints prohibiting field checking to confirm whether tonal contrasts visible on aerial photography were the result of fenceline contrasts or other factors. To map confirmed fencelines and not other, unconfirmed fencelines would give a false picture of the extent of fencing in the study area. 
Water developments are commonplace in the study area. Dugouts have been constructed in some ephemeral wetlands and ephemeral drainages on upland areas. Occasional dugouts are found in close association with riparian areas in Medicine Lodge Coulee. Reservoirs are numerous in the study area, ranging from major reservoirs such as Michel Reservoir in Section 5 - Twp. 7 - Rge. 3 - W4M, to many small reservoirs created as a result of earth dams within many ephemeral and permanent drainage channels in the study area. Though the reservoirs are man-made, they can have important wildlife values associated with them, particularly as water sources and in the provision of habitat for wildlife species which require habitat conditions which can exist around watering areas, such as sparsely vegetated, trampled areas for example. As well, drainage canals have been constructed to drain some relatively large wetland areas located primarily in meltwater channels at the base of the Cypress Hills. A few small wetlands have been drained on upland areas.

An extensive road and trail network has been developed throughout the area in response to agricultural developments, cattle grazing and oil and gas activities. Approximately $106 \mathrm{~km}$ of roads and trails occur within the study area. Of this, there are approximately $61 \mathrm{~km}$ of major roads, including the paved Highway 41 and gravelled, municipality-maintained allweather roads. In addition, $18 \mathrm{~km}$ of minor roads were mapped in the study area. Minor roads include gravel access roads leading to wellsites or farmsites. Approximately $27 \mathrm{~km}$ of vehicle trails on mineral soil were mapped as well. Cattle trails, while not mapped, are readily apparent on the air photos.

A number of pipelines were mapped within central portions of the study area. These pipelines total approximately $19 \mathrm{~km}$ in length and appear to be revegetated. Four wellsites and two reclaimed wellsites were mapped within the study area. 
Five major pits have been identified within the study area, from which clays and gravels are being extracted.

In addition to the above disturbances, a cemetary exists within NE 20-7-3-W4M, and a bible camp is located in NE 28-7-3-W4M. 


\subsection{CHECKLISTS OF SIGNIFICANT FEATURES}

The following are descriptions or checklists of mappable significant features identified in the Eagle Butte Sensitive Area. Locations of these sites are presented in Map 3. As well, an overall sensitivity rating is given for each site or feature.

The significant features checklists are grouped by level of significance, from national to local significance. 
Site Name:

Cypress Hills (Map Unit CH)

Site Location: central and eastern portions of the study area, east and north of Medicine Lodge Coulee.

\section{Description:}

a complex which includes portions of the Cypress Hills Plateau, the western and northern flanks of the Cypress Hills including the eastern slopes of Medicine Lodge Coulee, bedrock-controlled upland areas north and east of Medicine Lodge Coulee, and meltwater channels at the base of the northern escarpment of the Cypress Hills within which occasional ephemeral and permanent wetland occur, including Elkwater Lake.

Cypress Hills Plateau portions of the unit were unglaciated by Laurentide glacial advances. Plateau is capped by the Cypress Hills Formation, a conglomerate of Tertiary gravels.

- numerous geological formations are exposed along the flanks of the Cypress Hills, more so than found in the remainder of southeastern Alberta.

numerous fresh water springs and perched wetlands.

hundreds of disjunct plant and animal species, including relict rough fescue grasslands and forests with similarities to western Cordilleran forests

\section{Significance: National}

undisturbed grassland areas are part of one of the few remaining, relatively large, contiguous tracts of native prairie left in Canada.

- rare flora which occur include marsh butterweed (Senecio foetidus), rare in Canada, and American pellitory (Parietaria pensylvanica), pine-drops (Pterospora andromedea), tall meadow rue (Thalicatrum dasycarpum), low yellow evening-primrose (Oenothera 
flava) and small-flowered hawk's-beard (Crepis occidentalis), all of which are rare in Alberta. Many other rare plant species may occur as well.

the unit encompasses portions of critical winter habitat for elk, mule deer, white-tailed deer and moose.

swift fox sighted in southern parts of the unit near Read Creek, also in Cypress Hills Provincial Park adjacent to the study area.

burrowing owl, classed as "Threatened" by COSEWIC and "Endangered Animal" by Alberta Fish and Wildlife Services, nesting in southeastern portion of the unit on uplands in the vicinity of Read Creek.

habitat for northern leopard frog, a species "Red-listed" by Alberta Fish and Wildlife Services, in the Sexton Creek area is regarded as the best in the province.

hibernacula for red-sided garter snakes occur in northern portion of the unit, immediately north of Cypress Hills Provincial Park.

sharp-tailed grouse leks on grassland plateaus above Sexton Creek.

two western painted turtles, classed as a "Blue-listed" species by Alberta Fish and Wildlife Services, occur in beaver pond near Sexton Creek.

one of only two areas in the study area providing habitat for introduced populations of red squirrel.

includes Eagle's Nest Candidate Natural Area, within which many of the ablove features are represented.

Sensitivity: $\quad$ Moderate to Very High 


\section{Comments:}

significant amount of cultivation exists in southern portions of the unit on the Cypress Hills Plateau, which devalues those portions as important examples of native mixed grass prairie. However, these areas can still be considered as hydrogeologically important due to the underlying conglomerate of the Cypress Hills Formation. Precipitation falling on the plateau percolating through the conglomerate recharges groundwater which discharges in numerous springs along the flanks of the Cypress Hills. As such, development activities on the Cypress Hills Plateau could have implications with regard to their effect on groundwater quality.

rough fescue grasslands are particularly sensitive to heavy grazing pressures.

\section{References:}

- 1995 field investigations

- Packer and Bradley (1984), Wallis (1991, 1990), Biota Consultants Ltd. and Komex International (1992) for rare flora and fauna

Roed (n.d.), Westgate (1968), Borneuf (1976) for geology and hydrogeology

Breitung (1954), Newsome and Dix (1968), Coupland and Brayshaw (1953), Coupland (1961), Moss and Campbell (1947) for vegetation Alberta Fish and Wildlife Division (1991)

COSEWIC (1995)

Willoughby (1992), Willms et al. (1985) for grazing responses

LANDSAT-5 TM image 52763-173550 September 24, 1991 
Site Name:

Site Location:

\section{Eagle Butte Uplands (Map Unit EBU)}

western portions of the study area west of Medicine Lodge Coulee.

\section{Description:}

consists of bedrock-controlled uplands, including Eagle Butte, west of Medicine Lodge Coulee, and western slopes of Medicine Lodge Coulee.

geologically diverse, with economically important formations such as the Whitemud Formation from which clay is mined for industrial ceramic production.

conglomerate capping Eagle Butte is lithologically similar to conglomerate of the Cypress Hills Formation, but is of a different age and not part of the Cypress Hills Formation.

hundreds of disjunct plant species, including relict rough fescue grasslands and forests with similarities to western Cordilleran forests

pennyroyal (Hedeoma hispidum), a plant species which is rare in Alberta, occurs in the unit.

critical winter and summer range for elk, mule deer, white-tailed deer and moose. sharp-tailed grouse leks on upland.

one of only two areas in the study area providing habitat for introduced populations of red squirrel (along with hills north of Spruce Coulee Reservoir, all others are within Cypress Hills Provincial Park).

nesting areas for Baird's sparrow, a species classed as "Red-listed" by Alberta Fish and Wildlife Services.

\section{Significance: National}

undisturbed grassland areas are part of one of the few remaining, relatively large, contiguous tracts of native prairie left in Canada. 
Sensitivity: $\quad$ Moderate to Very High

\section{Comments:}

rough fescue grasslands are particularly sensitive to heavy grazing pressures.

\section{References:}

- 1995 field investigations

- Packer and Bradley (1984), Wallis (1991, 1990), Biota Consultants Ltd. and Komex International (1992) for rare flora and fauna

- $\quad$ Roed (n.d.), Westgate (1968), Borneuf (1976) for geology and hydrogeology

- Breitung (1954), Newsome and Dix (1968), Coupland and Brayshaw (1953), Coupland (1961), Moss and Campbell (1947) for vegetation

Alberta Fish and Wildlife Division (1991)

COSEWIC (1995)

Willoughby (1992), Willms et al. (1985) for grazing responses

LANDSAT-5 TM image 52763-173550 September 24, 1991 
Site Name: $\quad$ Hummocky Moraine (Map Unit HM)

Site Location: $\quad$ extreme northern portion of the study area.

\section{Description:}

includes areas of hummocky disintegration moraine, with minor areas of ridged end moraine, immediately north of the complex of bedrock-controlled uplands, meltwater channels and northern slopes of the Cypress Hills which comprise the Cypress Hills unit in the study area.

numerous ephemeral and permanent wetlands within depressions.

significant for waterfowl and shorebird staging habitats in the study area, providing the only complexes of standing water, even if only ephemeral, in the study area.

sharp-tailed grouse leks north of Elkwater Lake.

nesting areas for Baird's sparrow, a species classed as "Threatened" by COSEWIC and "Red-listed" by Alberta Fish and Wildlife Services.

\section{Significance: National}

undisturbed grassland areas are part of one of the few remaining, relatively large, contiguous tracts of native prairie left in Canada.

Sensitivity: $\quad$ Moderate to High

\section{References:}

1995 field investigations

Packer and Bradley (1984), Wallis (1991, 1990), Biota Consultants Ltd. and Komex International (1992) for rare flora and fauna

Roed (n.d.), Westgate (1968), Borneuf (1976) for geology and hydrogeology 
- Breitung (1954), Newsome and Dix (1968), Coupland and Brayshaw (1953), Coupland (1961), Moss and Campbell (1947) for vegetation

- $\quad$ Alberta Fish and Wildlife Division (1991)

- $\quad$ COSEWIC (1995)

- $\quad$ LANDSAT-5 TM image 52763-173550 September 24, 1991 
Site Name: Medicine Lodge Coulee - Lodge Creek (Map Unit MLC-LC)

Site Location: dissecting the southwestern portion of the study area.

\section{Description:}

consists of the riparian areas and floodplain of Lodge Creek and its tributaries within southern portions of Medicine Lodge Coulee in the study area.

- $\quad$ Lodge Creek is part of the Missouri River drainage system.

- $\quad$ sharp-tailed grouse leks exist in unit.

- critical mule deer summer and winter ranges.

pronghorn antelope habitat in extreme southern portion of Lodge Creek in the study area.

\section{Significance: $\quad$ Provincial}

riparian areas of Lodge Creek and an un-named tributary leading to Michel Reservoir provide habitat for northern leopard frog, a species "Red-listed" by Alberta Fish and Wildlife Services and confirmed in the unit.

Sensitivity: $\quad$ Moderate to High

\section{Comments:}

- Riparian areas are among the only sources of well-developed, vertically structured vegetation communities in lower elevation grasslands in the study area, providing valuable habitat for many species of fauna.

portions of the Lodge Creek floodplain have been disturbed due to cultivation and farmsites. 


\section{References:}

1995 field investigations

Wallis (1991)

Alberta Fish and Wildlife Division (1991) 
Site Name:

\section{Medicine Lodge Coulee - Bullshead Creek (Map Unit MLC-BC)}

Site Location: dissecting the northwestern portion of the study area.

\section{Description:}

consists of the headwaters, riparian areas and floodplain of Bullshead Creek within northern portions of Medicine Lodge Coulee in the study area.

Bullshead Creek is part of the South Saskatchewan drainage system.

some mule deer and white-tailed deer winter use but this is likely more significant in the upper reaches of Bullshead Creek.

Wallis (1991) cited ferruginous hawk and golden eagle nests but, again, this is for the upper reaches.

\section{Significance: Regional}

mousetail (Myosurus aristatus), a plant species rare in Alberta, occurs in undisturbed portions of the unit on saline alluvial fans.

unit includes the headwaters of Bullshead Creek.

wildlife use is limited due to the high level of impact resulting from agricultural disturbance.

Sensitivity: $\quad$ Low to High

Comments:

Riparian portions of unit are considered highly sensitive to disturbance, floodplain portions generally rated as having low sensitivity. 
- Riparian areas are among the only sources of well-developed, vertically structured vegetation communities in lower elevation grasslands in the study area, providing valuable habitat for many species of fauna.

- $\quad$ significant portions of the Bullshead Creek floodplain have been disturbed due to cultivation and farmsites.

\section{References:}

- 1995 field investigations

- Wallis (1991)

- Westgate (1968)

- $\quad$ Borneuf (1976) 


\section{Site Name: $\quad$ Manyberries Creek Valley (Map Unit MCV)}

Site Location: dissecting the extreme southwestern portion of the study area.

\section{Description:}

consists of the headwaters, riparian areas and floodplain of Manyberries Creek in the southwestern corner of the study area.

Manyberries Creek is part of an internal drainage system centred on Pakowki Lake.

\section{Significance: Local}

unit includes the headwaters of Manyberries Creek

wildlife use is limited due to the high level of impact resulting from agricultural disturbance.

Sensitivity: $\quad$ Moderate to High

\section{Comments:}

Riparian portions of unit are considered highly sensitive to disturbance, floodplain portions generally rated as having moderate sensitivity due to salinity.

significant portions of the Manyberries Creek floodplain have been disturbed due to cultivation and farmsites.

\section{References:}

1995 field investigations

Westgate (1968)

Borneuf (1976) 


\subsection{DATA GAPS}

Significant features identification, particularly of rare flora and fauna, is best accomplished by intensive surveys designed for this purpose to identify populations in the field. Field time allocated for the project was insufficient to conduct a thorough investigation of such features. Based on results from 1995 field study and the work of previous researchers, the potential exists for additional significant biological or physical features to be identified and described within te study area.

Wildlife population surveys and habitat surveys encompassing the study area have been conducted by Alberta Fish and Wildlife Services. These surveys have consisted of aerial surveys and pellet group transects, focusing primarily on game species such as elk, mule deer, white-tailed deer and moose. However, there is currently a lack of radio-telemetry data for the analysis of elk movement patterns in the Cypress Hills area. As well, information is lacking regarding the genetic nature of the Cypress Hills populations of elk and moose. These populations can be considered as "island" populations, even though rare and sporadic movements of moose into the Milk River area have been reported. The moose population of Cypress Hills apparently originated from four individuals.

Long-term climate data is lacking for the Cypress Hills. A ten-year climate study begun by Holmes $(1970,1969)$ remained uncompleted due to his accidental death. One climate station is maintained on the Cypress Hills Plateau, near Highway 41 south of Cypress Hills Provincial Park. However, this station is only equipped for the collection of temperature data, including daily temperature and monthly maximum and minimum temperatures. As well, this data is in "raw", unanalysed form (R. Eade, pers. comm.). 
Recent soil survey information is lacking for the study area. The most recent soil survey which included the entire study area was conducted by Wyatt et al. (1941), though more recent soils information for minor portions of the study area has been collected by Greenlee (1981) and Leskiw (1990).

Published studies are also limited regarding prehistoric and palaeontological sites within the study area. However, the bedrock formations exposed in the Eagle Butte Sensitive Area are fossiliferous, and a referral to Resource Management Program Unit, Cultural Facilities and Historical Resources Division, Alberta Community Development would be prudent prior to site-specific development activities in the study area.

This study will therefore best be used as an initial source to "flag" potential areas of concern related to any proposed development activity such as for oil and gas exploration and development. Detailed field study will be required to confirm site-specific occurrences of significant flora, fauna, cultural and palaeontological resources within the Manyberries Sensitive Area. 


\subsection{LITERATURE CITED}

Achuff, P.L. 1992. Natural regions and subregions of Alberta - a revised classification for protected areas management. Alberta Parks Service, Alberta Tourism, Parks and Recreation and Natural and Protected Areas, Alberta Forestry, Lands and Wildlife, Edmonton. 85 pp.

Alberta Environmental Protection. 1994. Ecological land survey site description manual. Alberta Land and Forest Services and Canadian Forest Service, Edmonton. 166 pp.

---a---. 1993. Alberta plants and fungi - master species list and species group checklists. Pub. No.: Ref. 75.

AFWD (Alberta Fish and Wildlife Division). 1991. The status of Alberta wildlife. Alberta Forestry, Lands, and Wildlife. Fish and Wildlife Division. Edmonton, Alberta. 49 pp.

1990. Alberta's threatened wildlife - leopard frog. Alberta Forestry, Lands and Wildlife; Fish and Wildlife Division. Edmonton, AB. 4 pp.

Anonymous. 1992. Petroleum activity on native prairie - guidelines for surface disturbances. Prepared by Alberta Environment, Alberta Forestry, Lands and Wildlife, Alberta Energy, and Energy Resources Conservation Board. Calgary, Alberta. 9 pp.

Argus, G.W. and K.M. Pryer. 1990. Rare vascular plants in Canada: our natural heritage. Canadian Museum of Nature, Ottawa. 191 pp. + maps.

Barinaga, M. 1990. Where have all the froggies gone? Science 247: 1033-1034.

Beaty, C.B. 1975. The Landscapes of Southern Alberta: a regional geomorphology. University of Lethbridge. $95 \mathrm{pp}$.

Bentz, J.A and A. Saxena. 1993. Significant ecological features inventory of the Lesser Slave Lake Integrated Resource Planning Area. Prepared for Resource Information Division, Alberta Environmental Protection, Edmonton, Alberta by Geowest Environmental Consultants Ltd., Edmonton, Alberta. $128 \mathrm{pp}$.

Biota Consultants and Komex International Ltd. 1992. Eagles Nest Candidate Natural Area special features and range inventory, 1992. Draft report. Alberta Forestry, Lands and Wildlife, Land Information Services Division, Edmonton. 84 pp. 
Bishop, C.A. and K.E. Pettit (eds). 1992. Declines in Canadian amphibian populations: Designing a national monitoring strategy. Occ. Pap. No. 76. Canadian Wildlife Service. Ottawa, ON. 120 pp.

Blaustein, A.A. and D.B. Wake. 1990. Declining amphibian populations: A global phenomenon. Trends Ecol. Evol. 5: 203-204.

Bonnichsen, R. and S.J. Baldwin. 1978. Cypress Hills ethnohistory and ecology: a regional perspective. Archaeological Survey of Alberta Occasional Paper No. 10. Alberta Culture, Historical Resources Division, Edmonton. 87 pp.

Borneuf, D.M. 1976. Hydrogeology of the Foremost Area, Alberta. Report 74-4, Alberta Research Council, Edmonton. 26 pp. + maps.

Bradshaw, D.A., A. Saxena, D.J. O'Leary and J.A. Bentz. 1995. Biophysical overview, significant, sensitive and disturbance features of the Manyberries Badlands Sensitive Area. Prepared for Land Information Division, Alberta Environmental Protection by Geowest Environmental Consultants Ltd.

1994. Biophysical overview, significant, sensitive and disturbance features of the Manyberries Badlands Sensitive Area. Prepared for Land Information Division, Alberta Environmental Protection by Geowest Environmental Consultants Ltd.

Breitung, A.J. 1954. A botanical survey of the Cypress Hills. Can. Field. Nat. 68:55-92.

Case, R.M. 1983. Pocket gophers. In: Prevention and Control of Wildlife Damage. Great Plains Agricultural Council and University of Nebraska. Lincoln, NB.

Coady, J.W. 1982. Moose. Pages 902-922 in: Chapman, J.A. and G.A. Feldhamer (eds). Wild Mammals of North America. Johns Hopkins University Press. Baltimore, MD.

Coote, D.R. and W.W. Pettapiece. 1989. Wind erosion risk: Alberta. Land Resource Research Centre, Agriculture Canada, Research Branch. Publication 5255/B. Contribution No. 87-08. 11 pp. + map.

COSEWIC. 1995. Species at risk in Canada. Committee on the Status of Endangered Wildlife in Canada. Ottawa, ON. 
Coupland, R.T. 1961. A reconsideration of grassland classification in the Northern Great Plains of North America. J. Ecol. 49:135-167.

1950. Ecology of mixed prairie in Canada. Ecological Monographs 20(4): 271 -

315.

and T.C. Brayshaw. 1953. The fescue grassland in Saskatchewan. Ecology 34:386-405.

Dale, B.C. 1983. Habitat relationships of seven species of passerine birds at Last Mountain Lake, Saskatchewan. M.Sc. Thesis. University of Regina. Regina, SK. 98 pp.

Dale, B.C. 1993. Productivity of endemic grassland passerines in haylands. Pages 27-32 in: Holroyd, G.L., H.L. Dickson, M. Regnier, and H.C. Smith (eds). Proc. of the Third Prairie Conserv. and Endang. Spec. Wkshp. Nat. Hist. Occ. Paper No. 19. Provincial Museum of Alberta. Edmonton, AB.

DeSmet, K. and W.S. Miller. 1989. Report on the status of the Baird's sparrow in Canada. Prepared for the Committee on the Status of Endangered Wildlife in Canada. Ottawa, ON. $28 \mathrm{pp}$.

Dickinson, D. 1968. A preliminary survey of elk and moose populations in the Cypress Hills. Wildl. Invest. Prog. Rept. Alberta Department of Lands and Forests, Fish and Wildlife Division. Lethbridge, AB. 16 pp.

Dickinson, D. 1969. Forage resources and utilization in the Cypress Hills Provincial Park. Wildl. Invest. Prog. Rept. Alberta Department of Lands and Forests, Fish and Wildlife Division. Lethbridge, AB. 14 pp.

Dickinson, D. 1991. Wild ungulates of the Cypress Hills: A review of information. Prepared for Alberta Forestry, Lands and Wildlife; Fish and Wildlife Division. Edmonton, AB. $70 \mathrm{pp}$.

Downs, A. 1981. The Cypress Hills of Alberta and Saskatchewan. Frontier Series No. 22. Heritage House Publishing Co. Surrey, BC. 
Eagles, P. 1984. The Planning and Management of Environmentally Sensitive Areas. Longman: London and New York.

Ecoplans Limited. 1982. Forest and fire management plan for Cypress Hills Provincial Park, Alberta. Prepared for Alberta Recreation and Parks. Edmonton, AB.

Edwards, B.F. 1974. A resource planning study of the wildlife of Cypress Hills Provincial Park, Alberta. M.Sc. Thesis. University of Calgary. Calgary, AB. 137 pp.

Environment Canada. 1993. Canadian climate normals 1961-90: Prairie provinces. Environment Canada, Atmospheric Environment Service. 266 pp.

Epp, H.T. 1991. Ecological process management for maintaining biological diversity on the Canadian prairies. Pages 72-77 in: Holroyd, G.L., G. Burns, and H.C. Smith (eds). Proc. of the 2nd Enadang. Species and Prairie Conserv. Wkshp. Nat. Hist. Occ. Paper No. 15. Provincial Museum of Alberta. Edmonton, AB.

Fargey, P.J. and A.W.L. Hawley. 1989. Seasonal patterns of forage selection by wapiti in relation to land reclamation. AECV89-R3. Alberta Environmental Center. Vegreville, AB. $112 \mathrm{pp}$.

Greenlee, G.M. 1981. Soil survey of Cypress Hills, Alberta and interpretation for recreational use. Earth Sciences Report 80-4. Alberta Institute of Pedology Number M-78-1. Alberta Research Council, Edmonton. 68 pp. + maps.

Gudmundson, L.D. 1993. Wildlife population surveys of Cypress Hills Provincial Park and adjacent areas, 1992/93. Alberta Environmental Protection, Fish and Wildlife Division. Edmonton, $\mathrm{AB}$.

Gudmundson, L.D. 1995. Wildlife population survey of Cypress Hills Provincial Park and adjacent area. Alberta Forestry, Lands and Wildlife; Fish and Wildlife Division. Lethbridge, AB. $11 \mathrm{pp}$.

Gunson, J.R. 1991. Management plan for elk in Alberta. Wildlife Management Planning Series No. 8. Alberta Forestry, Lands and Wildlife. Edmonton, AB. 
Halladay, I.R. 1965. A general survey of the natural history of the Cypress Hills. Pages 37 54 in: The 15th Annual Field Conference Guidebook: Cypress Hills Plateau. Alberta Society of Petroleum Geologists. Edmonton, AB.

Herrero, S., C. Mamo, L. Carbyn, and M. Scott-Brown. 1991. Swift fox reintroduction into Canada. Pages 246-252 in: Holroyd, G.L., G. Burns, and H.C. Smith (eds). Proc. of the 2nd Endang. Spec. and Prairie Conserv. Wkshp. Nat. Hist. Occ. Pap. No. 15. Provincial Museum of Alberta. Edmonton, AB.

Holmes, R.M. 1970. "Oasis effects" caused by the Cypress Hills. in: Proceedings of the Third Forest Microclimate Symposium, Kananaskis Forest Experiment Station, Seebe, Alberta, September 23-26, 1969. Canadian Forestry Service, Department of Fisheries and Forestry, Alberta/Territories Region, Calgary, Alberta. pp. 159-180.

1969. A study of the climate of the Cypress Hills. Weather 24:324-330.

Hunt, H.M. and E.R. Wiltse. 1980. Autumn diets of elk in three areas of Saskatchewan. Blue Jay 38(2): $125-129$.

Hunter, M.L. 1990. Wildlife, Forests, and Forestry - Principles of Managing Forests for Biological Diversity. Prentice Hall. New Jersey. 370 pp.

Johnston, A., S. Smoliak and R.A. Wroe. 1975. Poisonous and injurious plants of Alberta. Alberta Agriculture, Edmonton. 60 pp.

Johnson, R., C. Ziebell, D. Patton, P. Folliott, and R. Hamre (eds). 1985. Proc. of the 1st North Amer. Riparian Conf., Tucson, Arizona. Gen. Tech. Rep. No. RM-120. United States Forest Service, Rocky Mountain Forest and Range Experiment Station. Fort Collins, CO.

Keith, R.C. 1977. A preliminary study of the winter ecology of elk in the Cypress Hills of Saskatchewan. M.Sc. Thesis. University of Regina. Regina, SK. 205 pp.

Kocaoglu, S.S. 1990. Physical land classification methodology. Alberta Forestry, Lands and Wildlife, Land Information Branch, Resource Inventory Section. 41 pp.

Kramer, A. 1971. Notes on the winter ecology of mule and white-tailed deer in the Cypress Hills, Alberta. Can. Field-Nat. 85(2): 141-145. 
Kufeld, R.C. 1973. Foods eaten by the Rocky Mountain elk. J. Range Manage. 26(2): 106113.

Lane, J. 1968. Baird's sparrow. Pages 745-765 in: Austin, O.L. (ed). Life Histories of North American Cardinals, Grosbeaks, Buntings, Towhees, Finches, Sparrows, and Allies. Dover Publications. New York, NY.

Lee, P.G. 1979. Resource partitioning by elk and cattle: Cypress Hills Provincial Park, Alberta. M.Sc. Thesis. University of Alberta. Edmonton, AB. 254 pp.

Leskiw, L. 1990. Biophysical analysis of the Cypress Hills recreation lease. Prepared for Resource Information Branch, Alberta Forestry, Lands and Wildlife by Can-Ag Enterprises Ltd. Edmonton. 20 pp. + map.

Lewis, G.M. and E.A. Johnson. 1980. A study of the ecology of grassland communities and the effect of grazing in the Cypress Hills Provincial Park, Alberta. Parts I and II. Prepared for Alberta Recreation and Parks, Provincial Parks Division.

Lombard North Group. 1973. Resource inventory: Cypress Hills Provincial Park. Alberta Department of Public Works and Alberta Department of Lands and Forests. Edmonton, AB.

Looman, J. 1983. 111 range and forage plants of the Canadian prairies. Agriculture Canada, Research Branch. Publication 1751. Supply and Services Canada: Ottawa. 255 pp.

Lothian, W.F. 1987. A brief history of Canada's national parks. Indian and Northern Affairs Canada, Parks Canada. Ottawa, ON. 156 pp.

MacAuley, D. 1995. Cypress Hills Provincial Park management plan. Alberta Environmental Protection, Alberta Parks Services. Vulcan, AB. 207 pp.

McCorquedale, B. 1965. Some particular aspects of the biota of the Cypress Hills. Pages 55-65 in: The 15th Annual Field Conference Guidebook, Cypress Hills Plateau. Alberta Society of Petroleum Geologists. Edmonton, AB.

Morgantini, L.E. and W.B. Russell. 1983. An assessment of three selected elk winter ranges in the Rocky Mountains region. Prepared by Wildland Resources Consultants Ltd. for Alberta Fish and Wildlife Division. Edmonton, AB. 265 pp. 
Moss, E.H. 1983. Flora of Alberta. Second edition. Revised by J.G. Packer. University of Toronto Press: Toronto. 687 pp.

and J.A. Campbell. 1947. The fescue grassland of Alberta. Can. J. Research 25:209-227.

1944. The prairie and associated vegetation of southwestern Alberta. Can. J. Research 22:11-31.

Newsome, R.D. and R.L. Dix. 1968. The forests of the Cypress Hills, Alberta and Saskatchewan, Canada. Amer. Midland Nat. 80(1):118-185.

Nietfeld, M.T. 1983. Foraging behavior of wapiti in the boreal mixedwood forest, central Alberta. M.Sc. Thesis. University of Alberta. Edmonton, AB.

Nietfeld, M.T., J. Wilk, K. Woolnough, and B. Hoskin. 1984. Wildlife habitat requirement summaries for selected wildlife species in Alberta. Alberta Energy and Natural Resources, Fish and Wildlife Division. Edmonton, AB.

O'Leary, D., J. Bentz, D. Ealey and A. Schwabenbauer. 1993. Inventory of environmentally sensitive and significant natural areas, City of Edmonton. Prepared for Planning and Development, City of Edmonton by Geowest Environmental Consultants Ltd., Edmonton, Alberta. 299 pp.

Packer, J.G. and C.E. Bradley. 1984. A checklist of the rare vascular plants in Alberta. Provincial Museum of Alberta Natural History Occasional Paper No. 5. Alberta Culture. 112 pp.

Public Lands Division. 1990. Prairie Conservation Action Plan implementation. Public Lands Division, Alberta Forestry, Lands and Wildlife, Edmonton.

Quinn, M.S. 1991. Conservation of herpetofauna in the Prairie Provinces. Pages 197-198 in: Holroyd, G.L., G. Burns, and H.C. Smith (eds). Proc. of the Second Endang. Spec. and Prairie Conserv. Wkshp. Nat. Hist. Occ. Paper No. 15. Provincial Museum of Alberta. Edmonton, $\mathrm{AB}$. 
Renecker, L.A. and R.J. Hudson. 1992. Morphology, bioenergetics, and resource use. Pages 187-214 in: Stelfox, J.B. (ed). Alberta's Hoofed Mammals: Their Ecology, Status, and Management. Line Pine Publishing. Edmonton, AB.

Ressler, B. (no date). Cypress Hills Provincial Park: Introduction to the guidebook. Alberta Recreation and Parks, Cypress Hills Provincial Park. Elkwater, AB. 57 pp.

Risser, P.G. 1988. Diversity in and among grasslands. Pages 176-180 in: Wilson, E.O. (ed). Biodiversity. National Academy Press. Washington, DC.

Roberts, W. 1981. What happened to the frogs? Alberta Nat. 11(1): 1-4.

Roberts, W. 1992. Declines in amphibian populations in Alberta. Pages 14-16 in: Bishop, C.A. and K.E. Pettit (eds). Declines in Canadian Amphibian Populations: Designing a National Monitoring Strategy. Occ. Pap. No. 76. Canadian Wildlife Service. Ottawa, ON.

Roed, M.A. (no date). Guide to the geology: Cypress Hills Provincial Park, Alberta, Canada. $39 \mathrm{pp}$.

Russell, A.P. and A.M. Bauer. 1993. The Amphibians and Reptiles of Alberta. University of Alberta Press. Edmonton, AB. 264 pp.

Salt, W.R. and J.R. Salt. 1976. The Birds of Alberta with Their Ranges in Saskatchewan and Manitoba. Hurtig Publishers. Edmonton, AB.

Scace, R.C. 1978. Again the oasis: A century of landscape change in the Cypress Hills. Pages 31-48 in: Davies, W.A. (ed). Nature and Change on the Canadian Plains. University of Regina, Canad. Plains Res. Cent. Regina, SK.

Seburn, D. 1993. Handbook for monitoring the amphibians of Alberta. Alberta Environmental Protection, Fish and Wildlife Services. Edmonton, AB. 64 pp.

Semenchuk, G.P. 1992. Atlas of Breeding Birds of Alberta. Federation of Alberta Naturalists. Edmonton, AB. 390 pp.

Shetsen, I. 1987. Quaternary geology, southern Alberta. Alberta Research Council, Edmonton. Map. 
Skovlin, J.M. 1982. Habitat requirements and evaluations. Pages 369-414 in: Thomas, J.W. and D.E. Toweill (eds). Elk of North America: Ecology and Management. The Wildlife Management Institute. Washington, DC.

Smith, W. 1993. Wildlife habitat and use study - Eagle Butte / Cypress Hills, Alberta. Prepared for Roan Resources Ltd. Calgary, AB. 35 pp.

Soper, J.D. 1964. The Mammals of Alberta. Government of Alberta, Queen's Printer. Edmonton, $\mathrm{AB}$.

Stewart, R.E. 1975. Breeding Birds of North Dakota. Tri-College Center for Environmental Studies. Fargo, ND. 295 pp.

Stubbendieck, J., S.L. Hatch and K.J. Hirsch. 1986. North American Range Plants. Third edition. University of Nebraska Press: Lincoln. 465 pp.

Tajek, J., W.W. Pettapiece and K.E. Toogood. 1985. Water erosion potential of soils in Alberta: estimates using a modified USLE. Agriculture Canada, Research Branch. Technical Bulletin No. 1985-29, Ottawa. 27 pp. + map.

U.S.D.A. 1937. Range Plant Handbook. Reprinted 1988. United States Department of Agriculture, Forest Service. Dover Publications, Inc.: New York. 816 pp.

Wallis, C. 1992. Significant features of the Cypress Hills. Pages 1-11 in: Dickinson, D., D.A. Gauthier, and B. Mutch (eds). Proc. of the Cypress Hills For. Manage. Wkshp. The Society of Grassland Naturalists. Medicine Hat, AB.

------. 1991. Environmentally significant areas of the M.D. of Cypress No. 1. Prepared for Alberta Forestry, Lands and Wildlife, Resource Information Branch and Southeast Regional Planning Commission, Medicine Hat by Cottonwood Consultants Ltd. 76 pp.

1990. Reconnaissance special features assessment SE 28 and NE 21-7-3-W4. Prepared by Cottonwood Consultants Ltd. for Natural and Protected Areas Program, Alberta Forestry, Lands and Wildiife, Edmonton. 7 pp.

1986. An overview of reptiles and amphibians in Alberta's grassland and parkland region. Prepared for World Wildlife Fund Canada. Toronto, ON. 
1979. Significant plants of the Cypress Hills. Species list. 6 pp.

C. Bradley, M. Fairbarns and V. Loewen. 1987. The rare flora of Alberta. Volume 3: Species summary sheets. Alberta Forestry, Lands and Wildlife, Edmonton. Publ. No. T/155.

and C. Wershler. 1980. A habitat and biogeographic analysis of the birds of the Cypress Hills. Unpubl. manuscript.

Wershler, C., 1990. Status of the Baird's sparrow in Alberta - 1989. Prepared for World Wildlife Fund Canada and Alberta Fish and Wildlife Division. Edmonton, AB.

Western Ecological Services. 1980. A grazing management plan for Cypress Hills Provincial Park. Prepared for Alberta Recreation and Parks, Provincial Parks Division. Edmonton, AB. $71 \mathrm{pp}$.

Westgate, J.A. 1968. Surficial geology of the Foremost - Cypress Hills area, Alberta. Research Council of Alberta, Bulletin 22. 122 pp. + maps.

Willoughby, M. 1992. Species diversity and how it is affected by livestock grazing in Alberta. Range Notes No. 13. Alberta Forestry, Lands and Wildlife, Public Lands Division. $3 \mathrm{pp}$.

Willms, W.D., S. Smoliak and J.F. Dormaar. Effects of stocking rate on a rough fescue grassland vegetation. J. Range Manage. 38:220-225.

Wishart, W. 1986. The Wainwright deer herd (1966-1984): A comparative study of whitetailed and mule deer. Alberta Fish and Wildlife Division. Edmonton, AB. 59 pp.

WWFC (World Wildlife Fund Canada). 1988. Prairie conservation action plan. World Wildlife Fund Canada. Toronto, Ontario.

Wyatt, F.A., J.D. Newton, W.E. Bowser and W. Odynsky. 1941. Soil survey of the Milk River Sheet. University of Alberta, College of Agriculture, Bulletin No. 36. 105 pp. + maps. 


\section{PERSONAL COMMUNICATIONS}

L. Carbyn, Wildlife Research Biologist, Canadian Wildlife Service, Edmonton.

J. Clark, Wildlife Technician, Alberta Fish and Wildlife Division, Lethbridge.

R. Eade, Meteorological Technician, Resource Data Division, Alberta Environmental Protection, Edmonton.

D. Eslinger, Wildlife Biologist, Alberta Fish and Wildlife Division, Medicine Hat.

T. Hood, Public Lands, Alberta Agriculture, Food and Rural Development, Medicine Hat.

J. Taggart, Wildlife Technician, Alberta Fish and Wildlife Division, Medicine Hat.

D. Wetzel, Alberta Community Development, Cultural Facilities and Historical Resources Division, Resource Management Programs Unit, Edmonton.

M. Woodhouse, Alberta Community Development, Cultural Facilities and Historical Resources Division, Resource Management Programs Unit, Edmonton. 


\section{APPENDIX A}

\section{SIGNIFICANT FLORAL FEATURES OF THE EAGLE BUTTE SENSITIVE AREA}





\section{APPENDIX A \\ SIGNIFICANT FLORAL FEATURES OF THE EAGLE BUTTE SENSITIVE AREA}

During the course of 1995 field study, five provincially rare plant species, as defined by Packer and Bradley (1984) and Wallis et al. (1987), two plant species with restricted or local distribution in Alberta, and three plant species representing range extensions, were found in the study area. However, this does not mean that other rare plant species do not occur in the Eagle Butte Sensitive Area. Successful identification of rare plant populations is best accomplished though intensive survey methods designed to achieve this purpose. Numerous rare or uncommon plant species have been found by researchers in the Cypress Hills area in general (Wallis 1991, 1990 and 1979, Biota Consultants and Komex International Ltd. 1992). Similar habitats exist in many cases within the study area, and it is reasonable to assume that intensive survey would locate further rare plant populations within the study area.

The following species list of flora found within Eagle Butte Sensitive Area, or which may occur in the study area, includes plants considered notable due to four criteria:

- $\quad$ nationally rare (Argus and Pryer 1990)

- $\quad$ provincially rare (Packer and Bradley 1984)

- $\quad$ restricted or local distribution in Alberta (Wallis 1990)

- range extension, based on distribution maps of Moss (1983), not reported in the Cypress Hills area by previous researchers as mentioned above

It is expected that this list is by no means complete, and detailed site investigations would be required to determine further occurrences of rare plant populations in the study area. 
However, the list does serve to illustrate the importance of the Eagle Butte Sensitive Area overall in providing habitat or potential habitat for a significant amount of rare plant species.

Occurrence or potential occurrence of each species is indicated as follows:

- confirmed within the study area by GEOWEST personnel during 1995 field studies

- confirmed within the study area by previous researchers

- found in the vicinity of the study area by previous researchers. May occur within the study area

\section{Nationally Rare Plants}

- Erigeron radicatus (dwarf fleabane) - may occur on xeric ridges and slopes.

- Halimolobos virgata (halimolobos) - may occur on eroded slopes and dry benches, or sandy alkaline soil.

- Lilaea scilloides (flowering-quillwort) - may occur on slough margins and mudflats or dry creek beds.

- Lomatium cous (biscuit-root) - may occur on xeric grassland slopes, particularly at higher elevations in the study area just below the Cypress Hills plateau. 
- Orchis rotundifolia var. lineata (round-leaved orchid) - may occur in moist woods in the vicinity of springs.

Senecio foetidus (marsh butterweed) - found by Wallis (1990) in Eagle's Nest Candidate Natural Area as occasional on moist valley slopes.

- Trisetum wolfii (awnless trisetum) - may occur in moist woods.

\section{Provincially Rare Plants}

- Crepis occidentalis (small-flowered hawk's-beard) - found by Wallis (1990) in Eagle's Nest Candidate Natural Area as a small stand of thirty plants on eroding slopes.

- Hedeoma hispida (pennyroyal) - found in the Coulee Slopes community type as occasional on an east-facing, well-drained, submesic lower slope of Medicine Lodge Coulee.

- Melica subulata (Alaska onion grass) - may occur in moist coniferous woods.

- Mimulus guttatus (yellow monkeyflower) - may occur in riparian areas and in the vicinity of springs.

- Myosurus aristatus (mousetail) - found in the Alluvial Fan \Floodplain community type in Medicine Lodge Coulee in the vicinity of Bullshead Creek, occurring as frequent on a subhygric, saline, sandy clay alluvial fan. 
- Oenothera flava (low yellow evening-primrose) - found by Wallis (1990) in Eagle's Nest Candidate Natural Area as five plants in eroding shales.

- Parietaria pensylvanica (American pellitory) - found within the Shrub Draw community type as frequent in association with a mesic round-leaved hawthorn (Crataegus rotundifolia) and wild red raspberry (Rubus idaeus) thicket transitional between forest and grassland on north slope of the Cypress Hills, north-east of Reesor Lake.

- Polygonum watsonii (Watson's knotweed) - may occur on moist meadows or flats.

- Pterospora andromedea (pine-drops) - found as few in a lodgepole pine-pine grass stand below the Cypress Hills Plateau, on a north-facing, well-drained, subxeric, sandy clay loam slope.

- Thalictrum dasycarpum (tall meadow rue) - found as occasional to frequent in mesic to subhygric balsam poplar stands on north-facing slopes and valley bottoms.

- Veronica catenata (water speedwell) - may occur in the vicinity of springs, spring-fed streams or muddy shorelines.

\section{Restricted or Local Distribution in Alberta}

- Arenaria congesta (rocky-ground sandwort) - ubiquitous within the study area on subxeric, well drained, south-facing slopes within the Eroding Slopes, Coulee Slopes, Native Grassland and Hummocky Grassland community types. Also found by Wallis 
(1990) as abundant along upland edge within the Eagle's Nest Candidate Natural Area.

- Crataegus douglasii (Douglas hawthorn) - also found by Wallis (1990) and Biota Consultants and Komex International Ltd. (1992) in Eagle's Nest Candidate Natural Area. Occurs on moist valley slopes in tall shrub communities, depressions and aspen forest.

- Veronica serpyllifolia (thyme-leaved speedwell) - found by Wallis (1990) in Eagle's Nest Candidate Natural Area as a large population alongside a creek.

\section{Range Extensions}

- Calla palustris (water arum) - found as few in the Ephemeral Wetland community type, in a hydric, brown moss-spiked water milfoil-pondweed stand at the margin of standing water.

- Opuntia fragilis (brittle prickly-pear) - found as occasional within the Alluvial Fan \Floodplain community type on a xeric, saline, sandy clay loam alluvial fan within Manyberries Creek Coulee.

- Ranunculus pensylvanicus (bristly buttercup) - found as few to occasional in the Riparian community type in subhygric to hygric, sandy clay loam, intermittent stream drainages 

APPENDIX B

PLATES 
- 


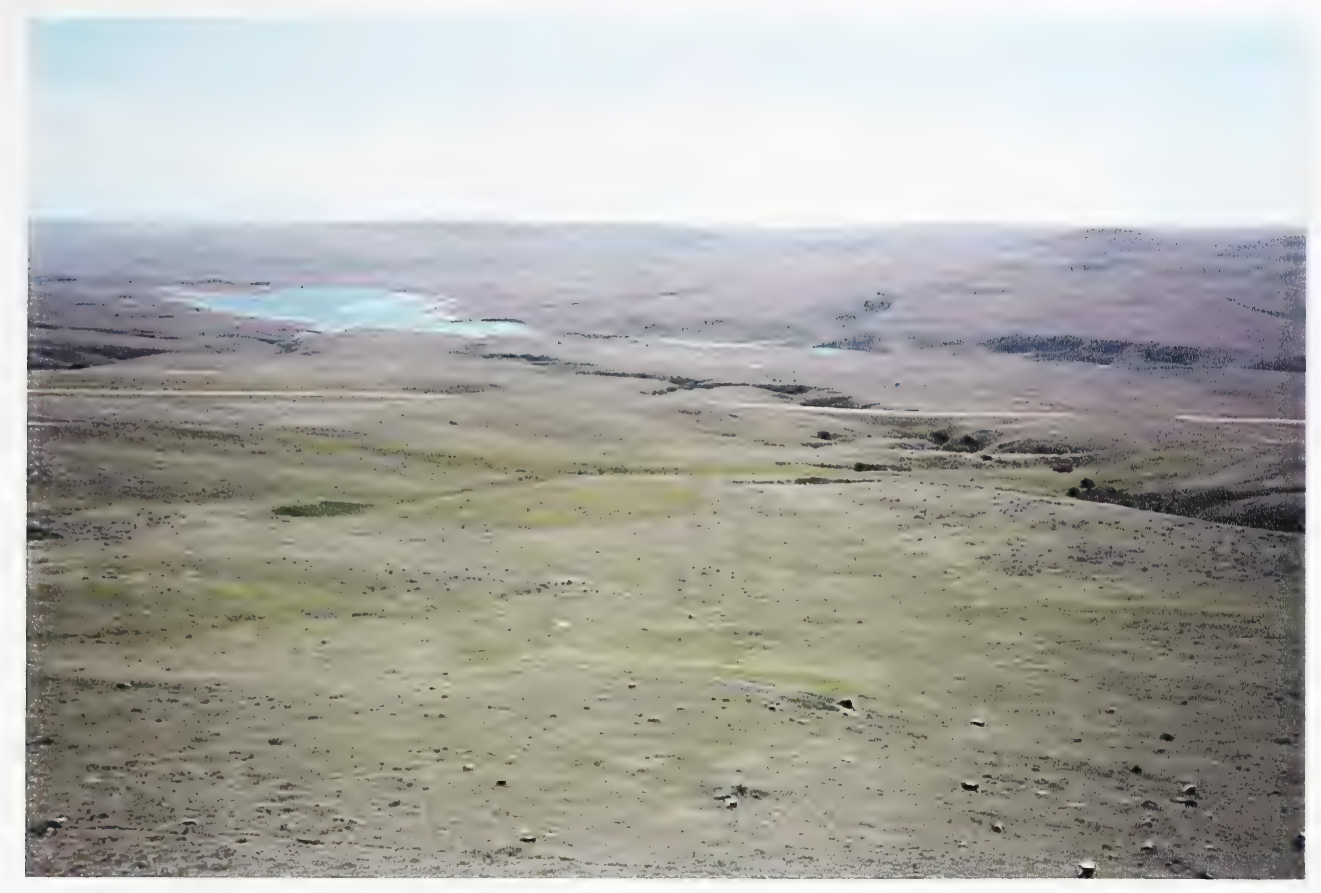

Plate 1: Mixed grass prairie in the southern portion of the study area within a tributary branch of Medicine Lodge Coulee, looking south. Much of the area in the photo represents the Coulee Slopes community type as it occurs on moderate slopes. The Shrub Draw community type can also be seen, occurring within the numerous ephemeral drainage draws which dissect the Coulee Slopes community type. Note heavy cattle grazing pressure here. The Native Grassland community type is extensive on uplands in the background.

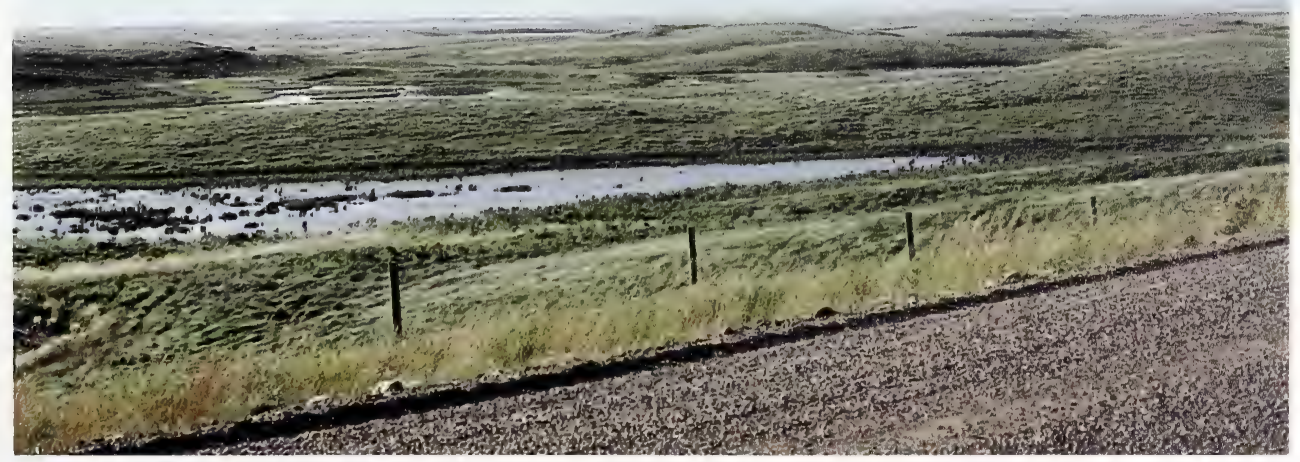

Plate 2: Hummocky Grassland community type on hummocky disintegration moraine which occurs extensively along the northern boundary of the study area. Ephemeral and permanent wetlands are common within depressional areas, providing significant waterfowl and shorebird staging habitats. 



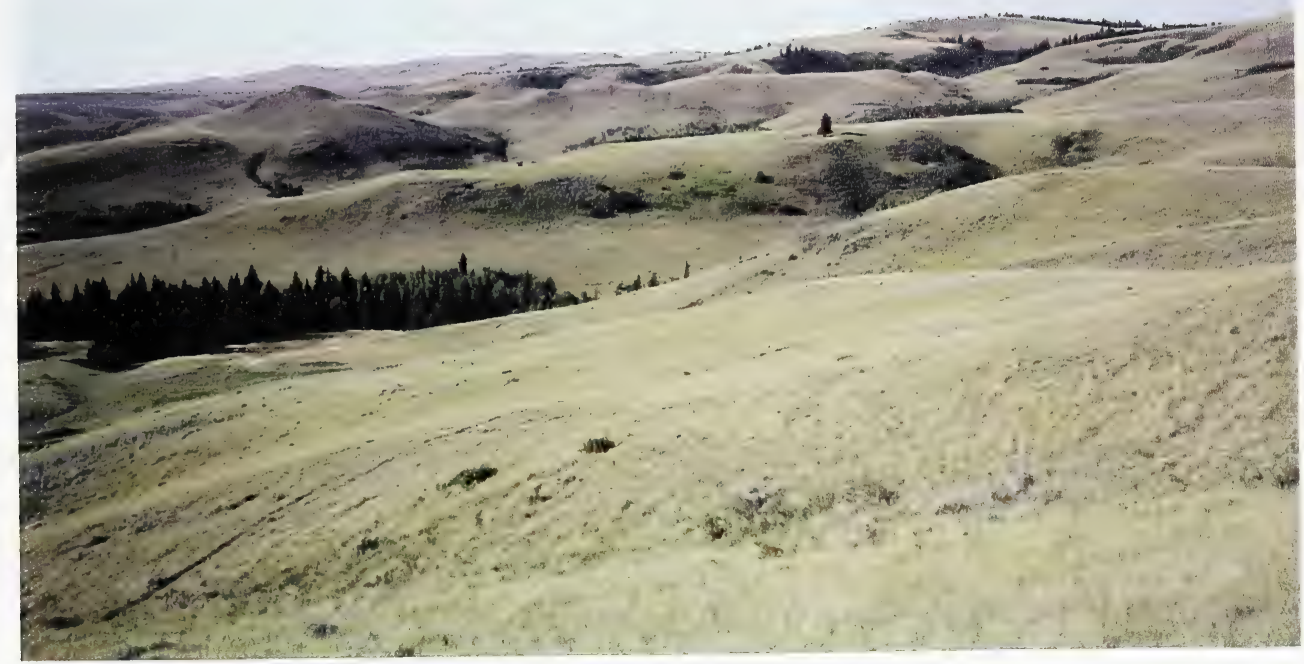

Plate 3: Bedrock-controlled uplands at higher elevations in the study area south of Eagle Butte, looking north-west. Complex topography and local climatic effects resulting from rapid elevational gradients in the Cypress Hills area contribute to a highly variable complex of moisture regimes which is reflected in the vegetation of the area. Forest communities can extend far into grassland areas along sheltered draws and riparian areas. Shrub communities, such as that visible at photo center, occur where moisture regimes are favourable due in part to aspect or groudwater seepage. Grassland communities here are transitional between mixed grass communities dominated by western porcupine grass (Stipa curtiseta) at lower elevations and the Festuca scabrella Association common to higher elevations.

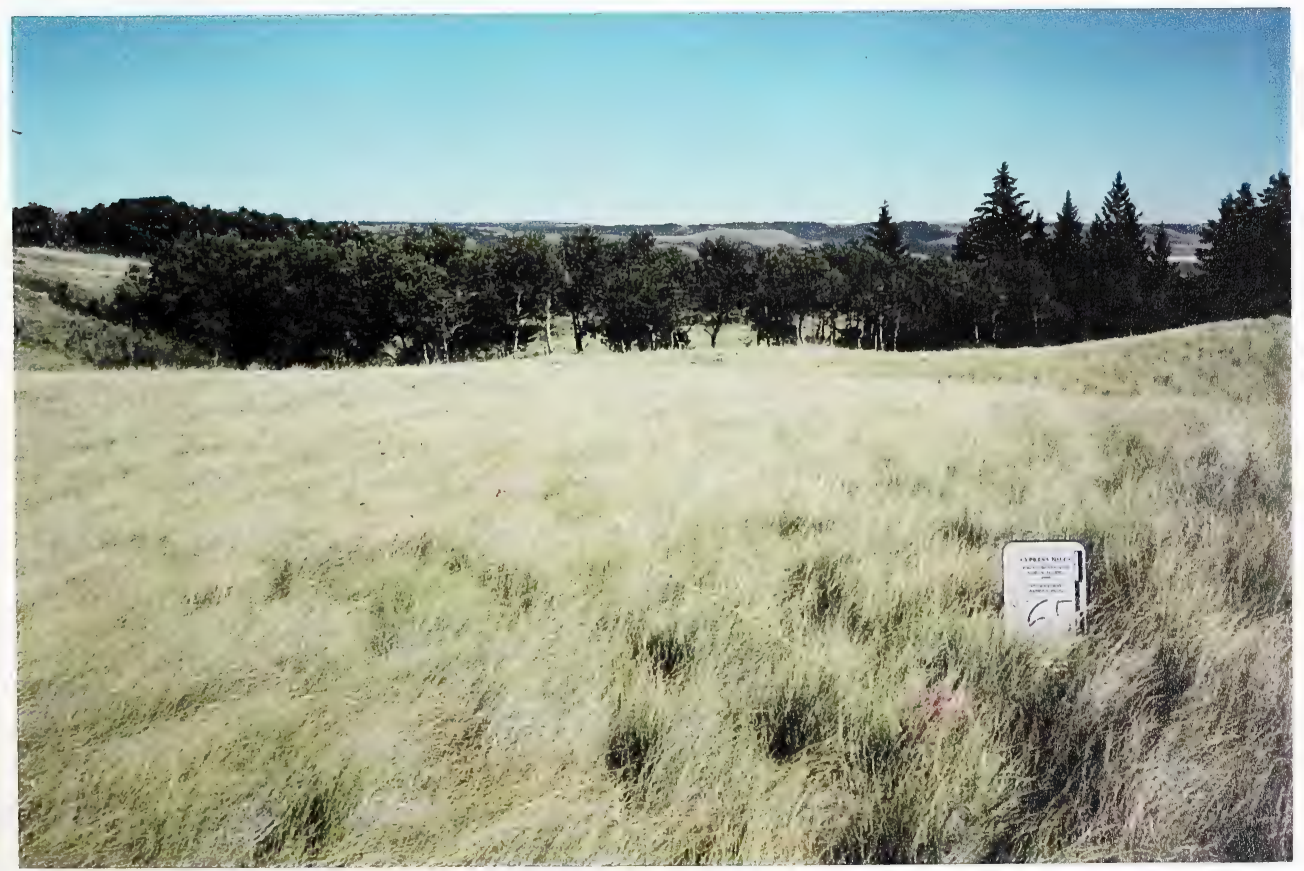

Plate 4: Rough fescue - western porcupine grass community in the vicinity of Eagle Butte, with aspen (Populus tremuloides) and white spruce (Picea glauca) forest communities in the background. Such grassland-forest mosaics are common in the study area, providing habitat diversity and significant edge habitat important for many wildlife species. 



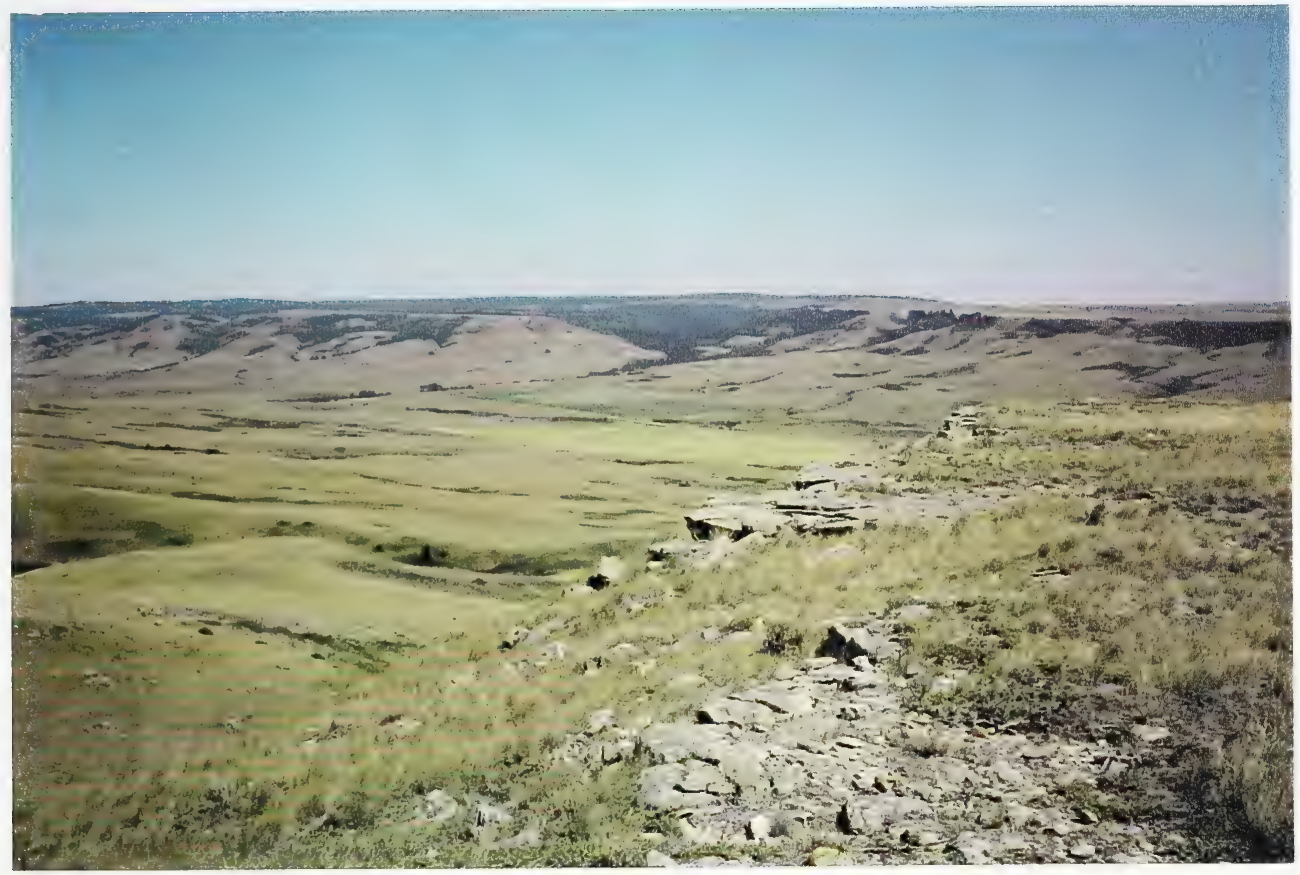

Plate 5: Medicine Lodge Coulee, looking north-east, with the Cypress Hills Plateau in the background. The Eroded Slopes community type (note bedrock outcrops) in the foreground intergrades here to the Coulee Slopes and Shrub Draw community types. Note cultivated areas within the coulee bottom, common in the study area.

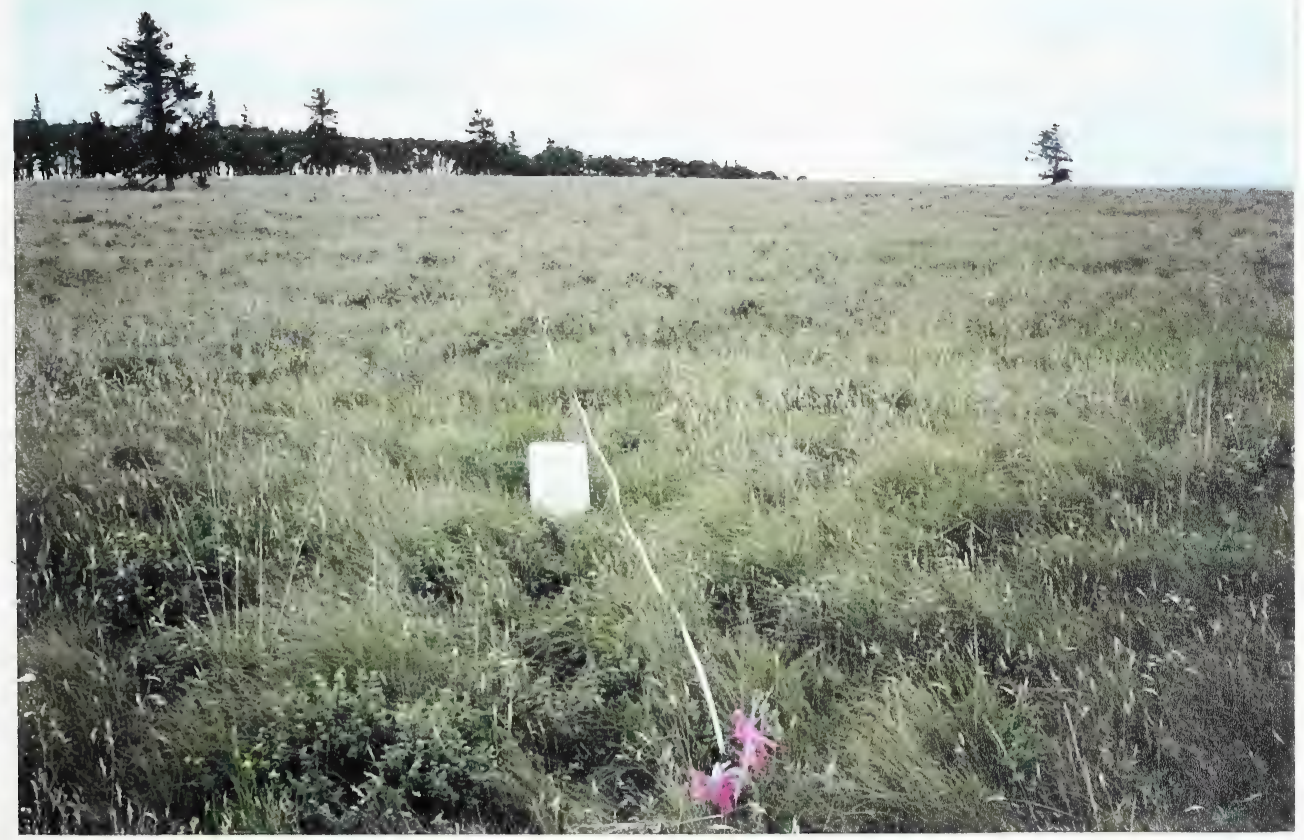

Plate 6: Native Grassland community type, in this case, a rough fescue - California oat grass (Festuca scabrella - Danthonia californica) community in the study area on the Cypress Hills Plateau near the southern boundary of Cypress Hills Provincial Park. Note the common occurrence of shrubby cinquefoil (Potentilla fruticosa), a shrub species characteristic of rough fescue grasslands in the Cypress Hills and in the foothills of south-western Alberta, but notably absent from rough fescue grasslands in the "aspen grovelands" of central Alberta and west-central Saskatchewan. 



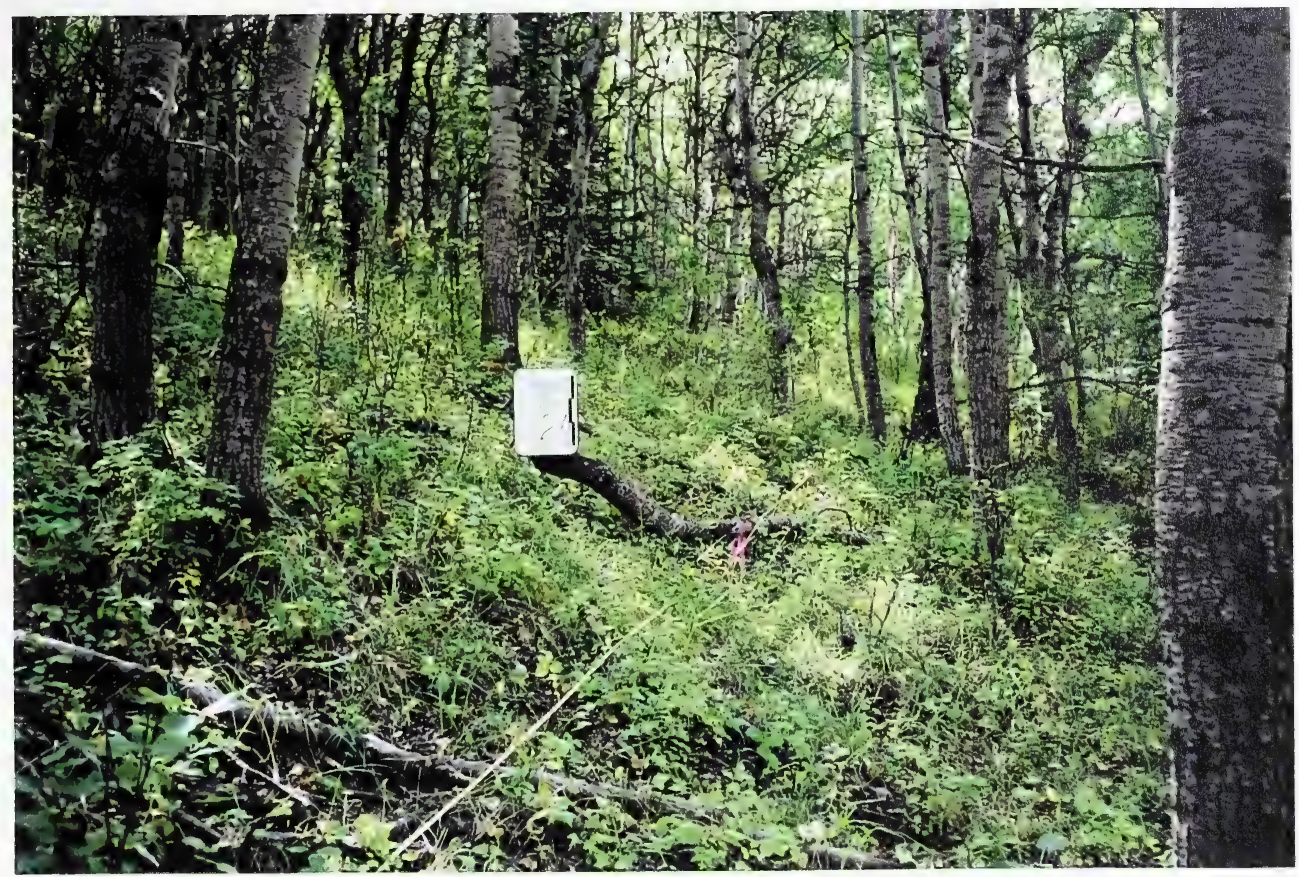

Plate 7: Mixed Wood community type, dominated at this site by aspen with regenerating white spruce in the background, located on north-facing slopes of the Cypress Hills in the northern portion of the study area. The well-developed vertical structure of such stands provide important food and cover values for wildlife.

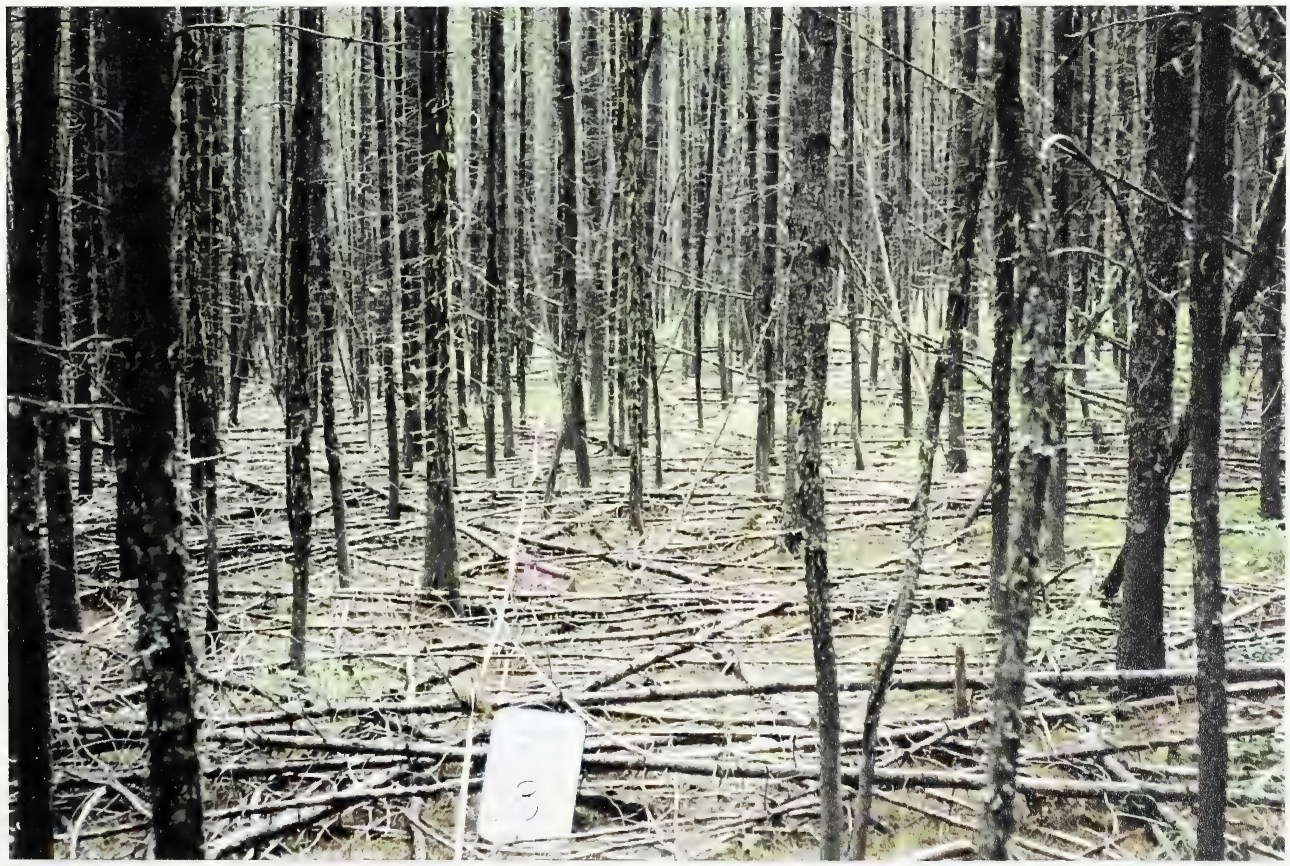

Plate 8: Lodgepole pine - pine reed grass (Pinus contorta - Calamagrostis rubescens) stand within the Coniferous Forest community type in the study area immediately south of Cypress Hills Provincial Park. Note the poorly-developed vertical structure of such stands as compared the mixed wood stand depicted in Plate 7. Pine-drops (Pterospora andromedea), rare in Alberta, occurs at this particular site. 



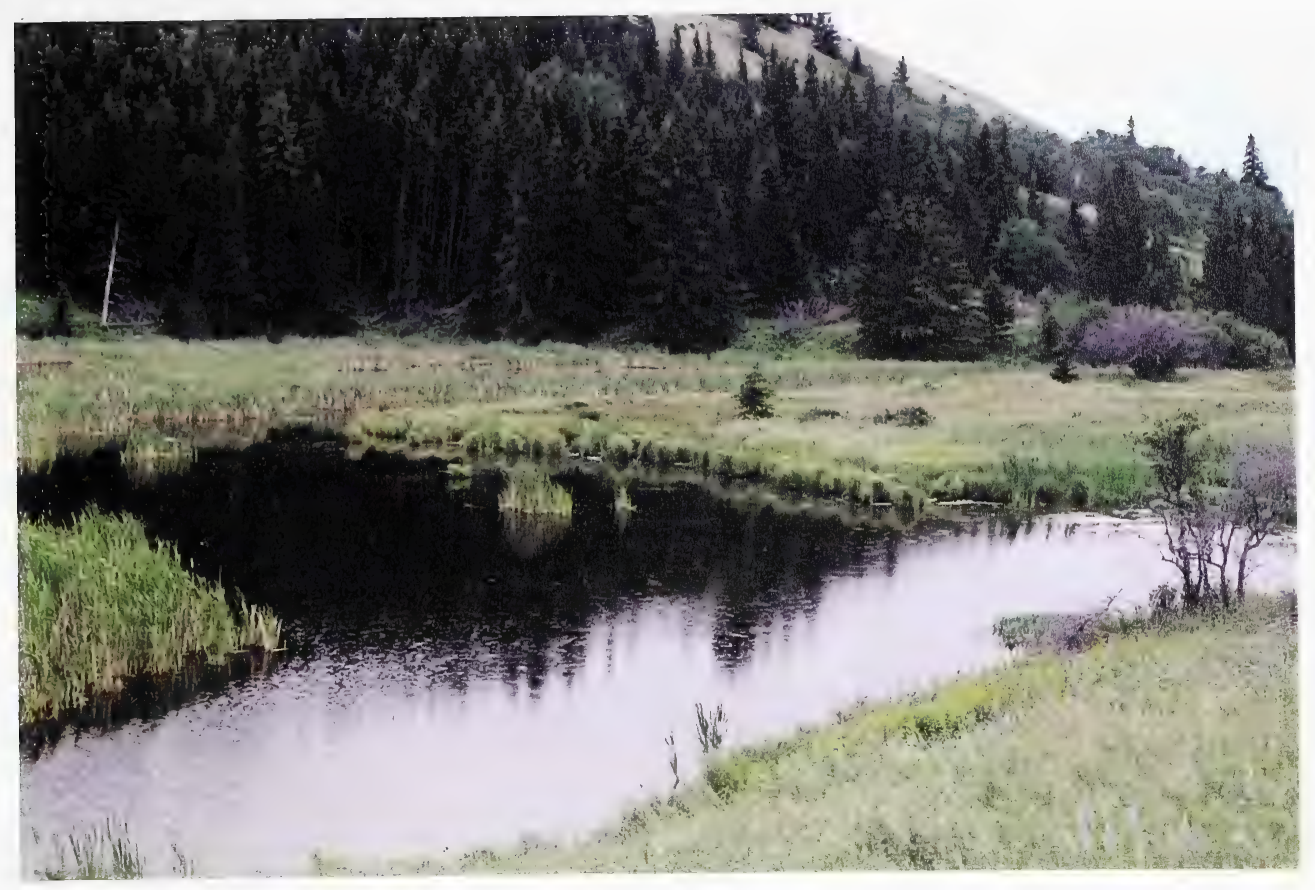

Plate 9: Wetland created by beaver activity. Many of the wetlands along the flanks of the Cypress Hills are spring-fed and provide important habitat for significant plant and animal species including northern leopard frog, a herptile classed as "Red-listed" by Alberta Fish and Wildlife Services (see Plate 10 below).

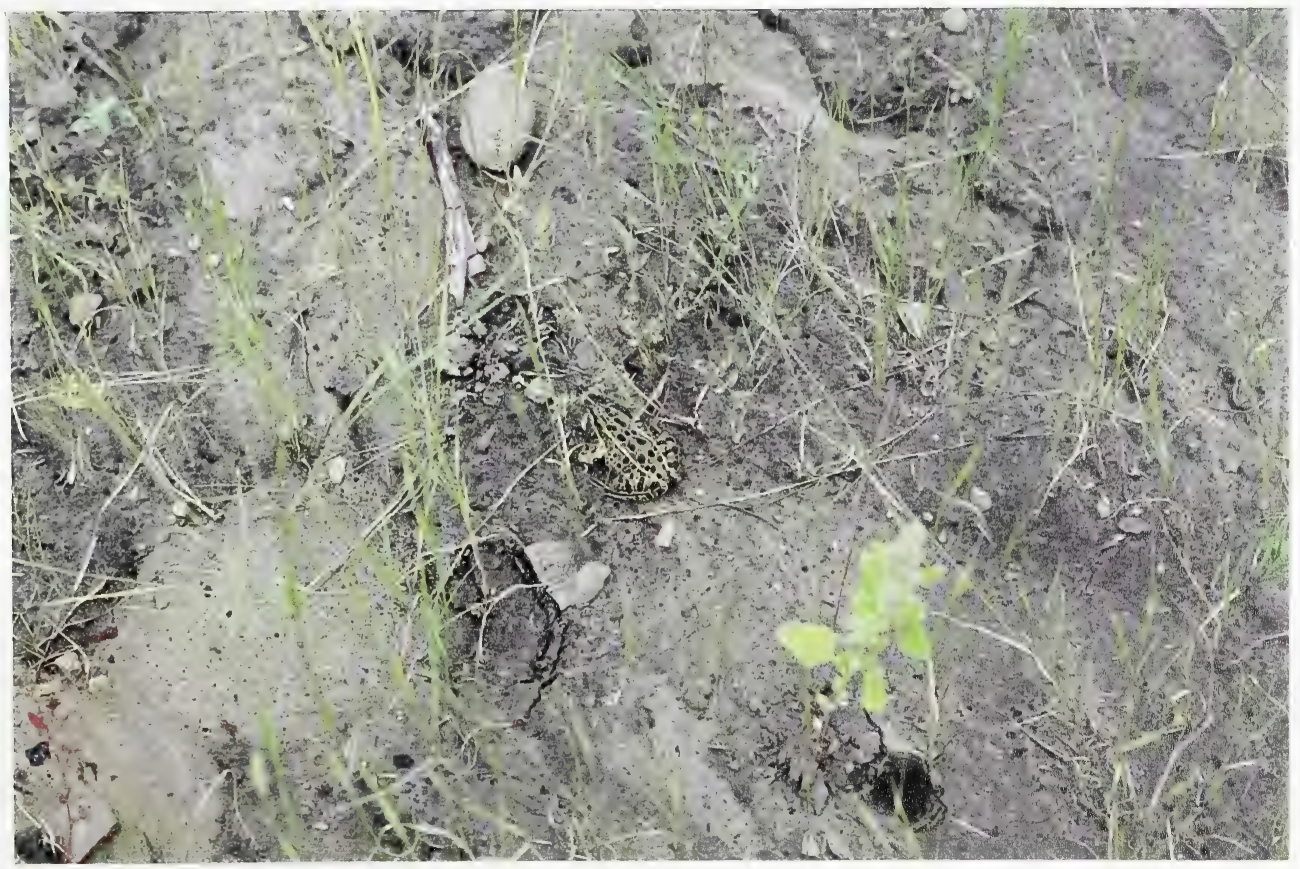





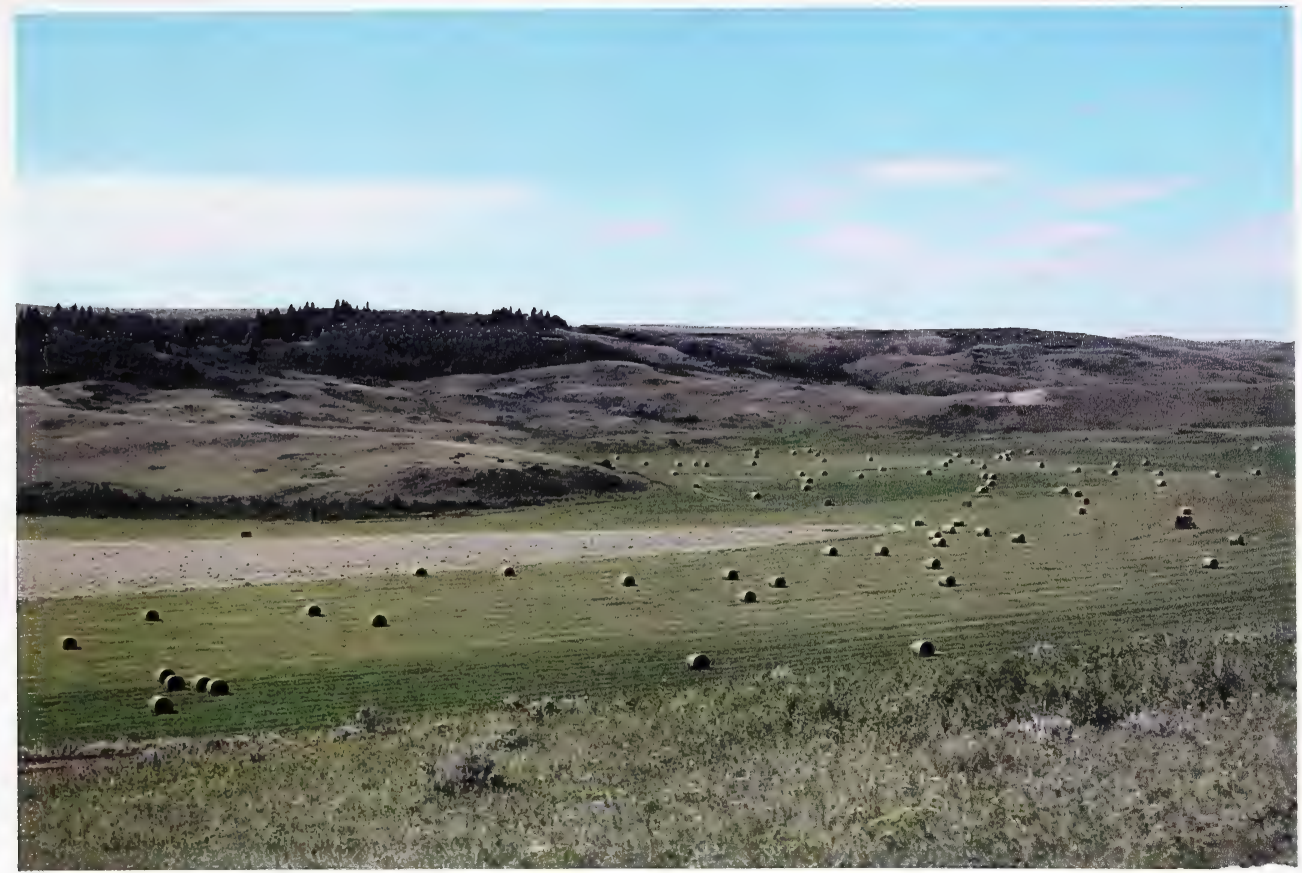

Plate 11: Haying on Fly Lake. Many large ephemeral wetlands are hayed in the study area. Drainage canals have often been constructed within them, including at Fly Lake.

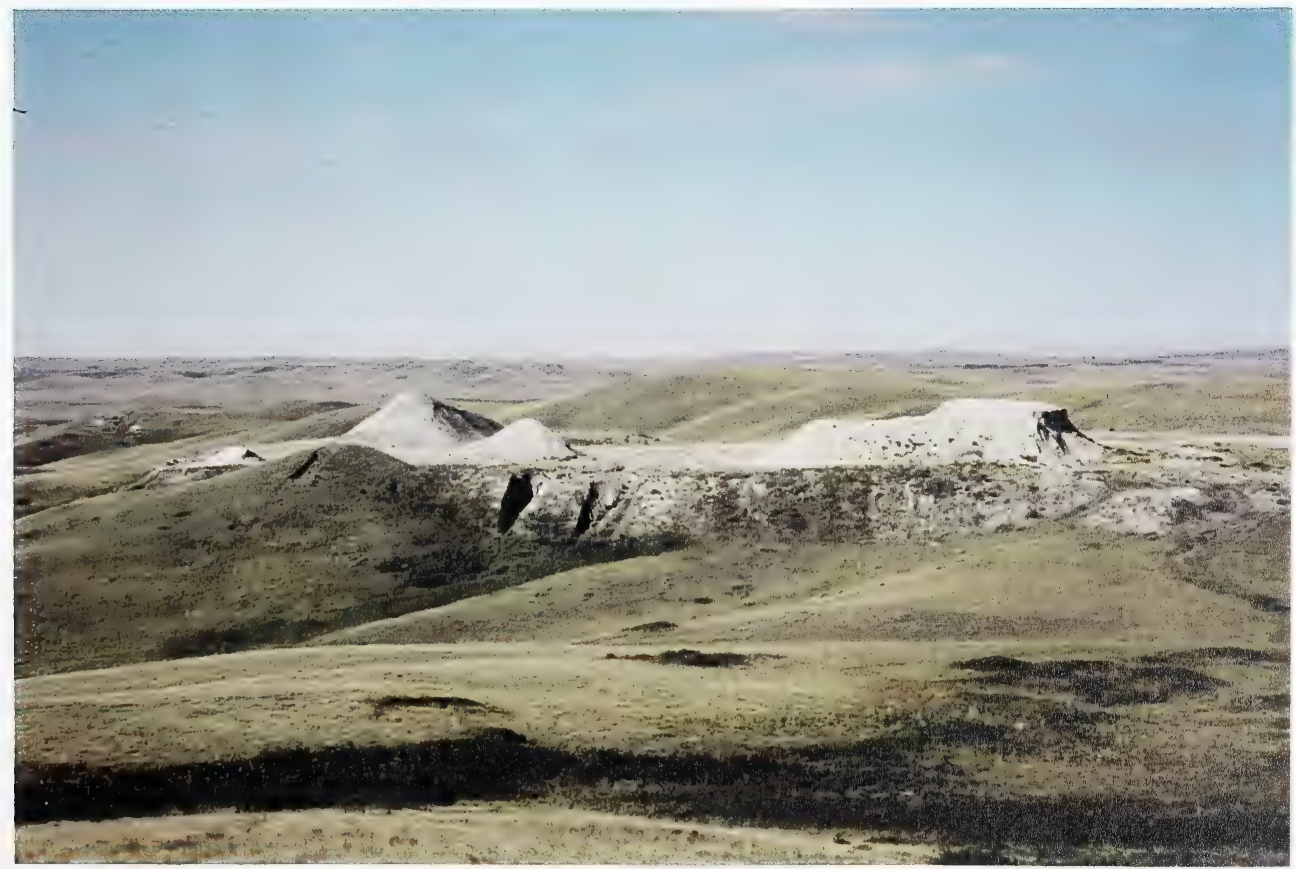

Plate 12: $\quad$ Quarry on bedrock-controlled uplands in the study area west of Medicine Lodge Coulee near the western boundary of the study area. In addition to the five major quarries present within the study area, other disturbance features include cultivation, water developments such as reservoirs and dugouts, roads and vehicle trails, wellsites and pipelines. Disturbances from oil and gas activity has been relatively minor in the study area when compared to other sensitive areas such as the Manyberries Badlands Sensitive Area, for example. 





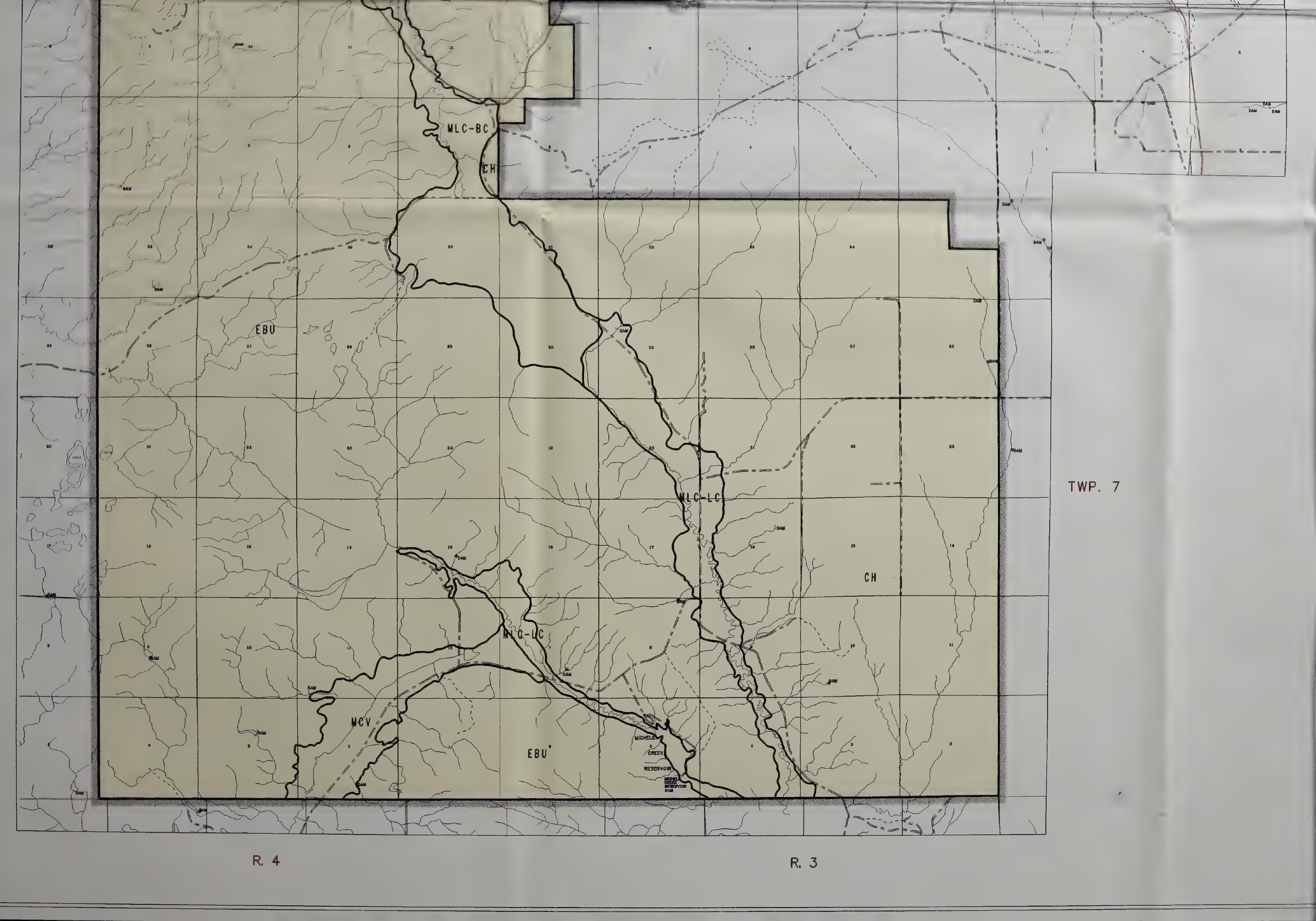


EAGLE BUTTE E.S.A.

SIGNIFICANT FEATURES MAP

SCALE $1: 50,000$

S I GNI F I CANT FEATURES

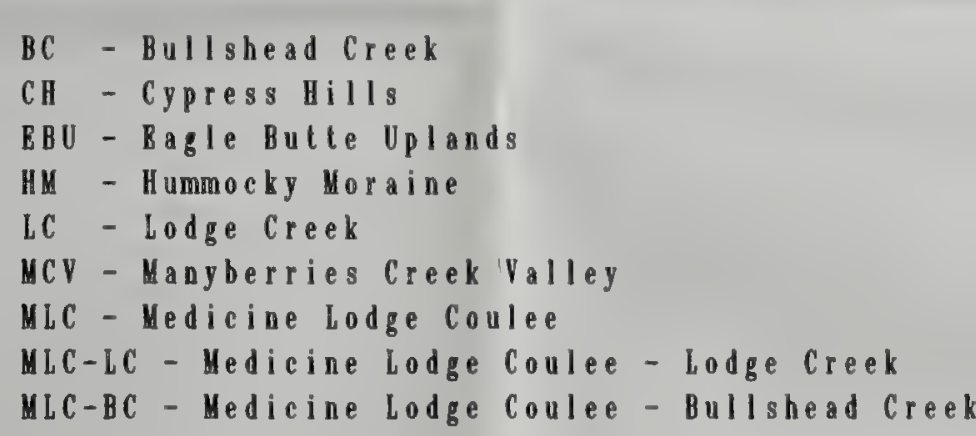

TOPOGRAPHIC BASE FEATURES

TWP. 7

\begin{tabular}{|c|c|}
\hline & $\begin{array}{l}\text { Major Highray } \\
\text { Secondary Highway }\end{array}$ \\
\hline & Roads \\
\hline & Truck Trails \\
\hline & Hydrography \\
\hline & Section Lines \\
\hline
\end{tabular}




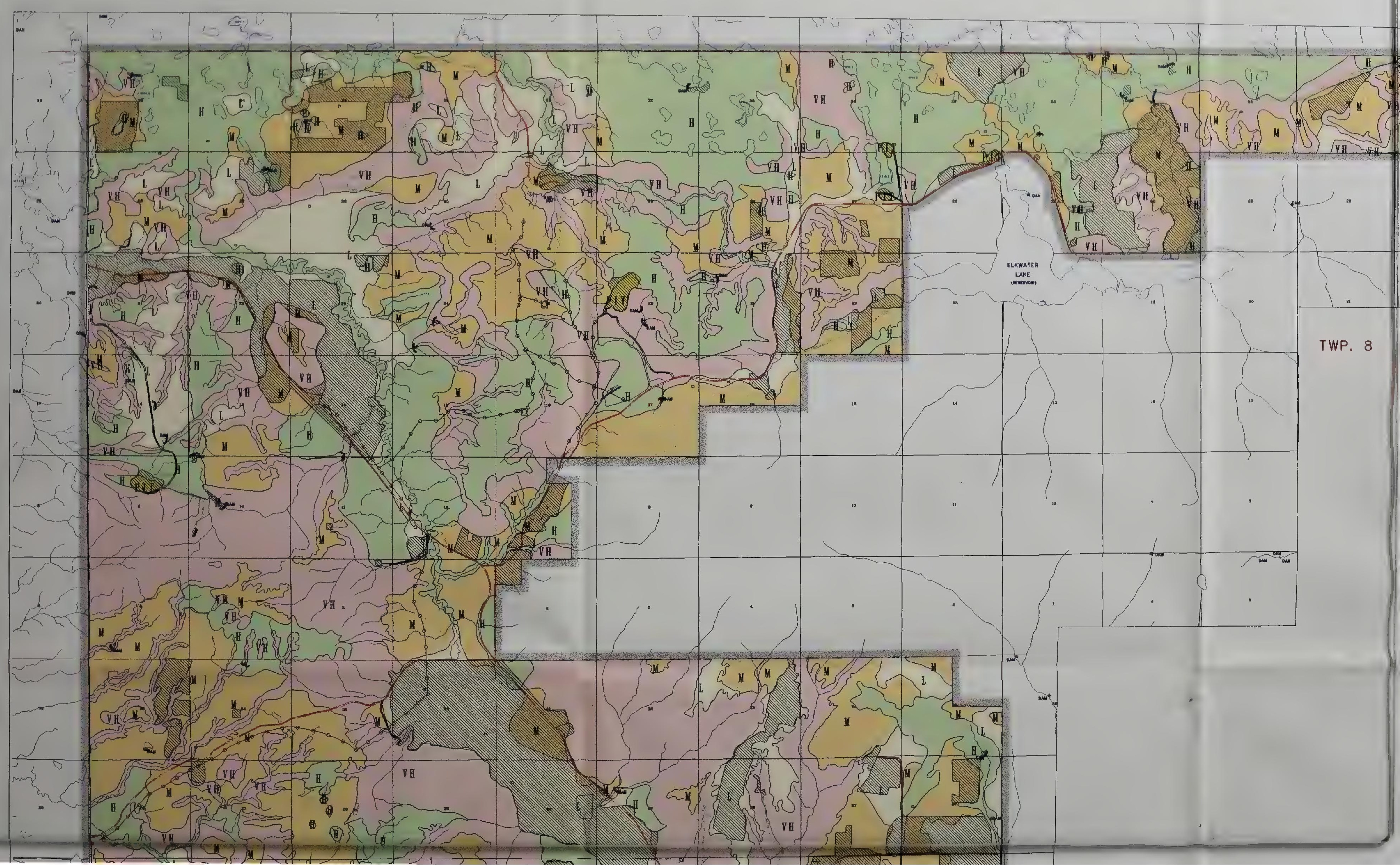




\section{EAGLE BUTTE E.S.A.}

SENSITIVE AREAS AND D ISTURBANCE MAP

D I STURBANCE FEATURES

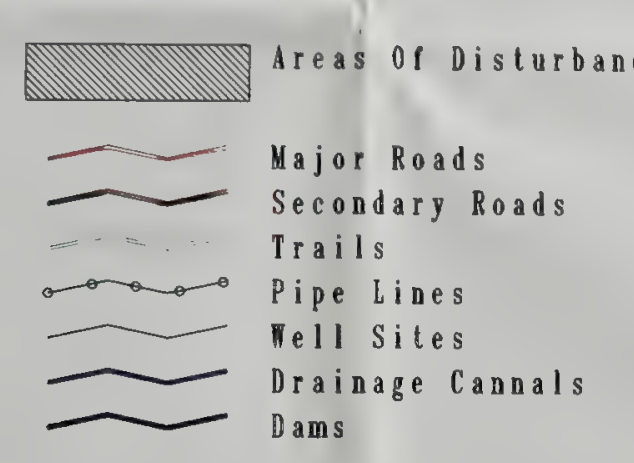

SENS I T I I TY RATINGS

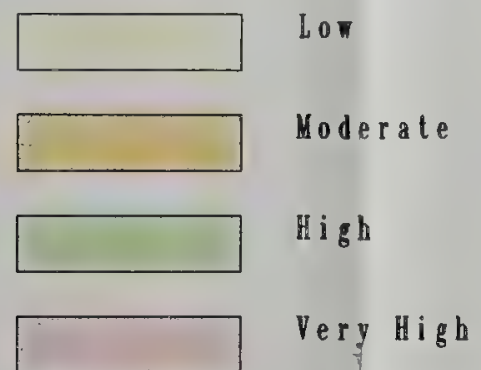

SCALE $1: 50,000$

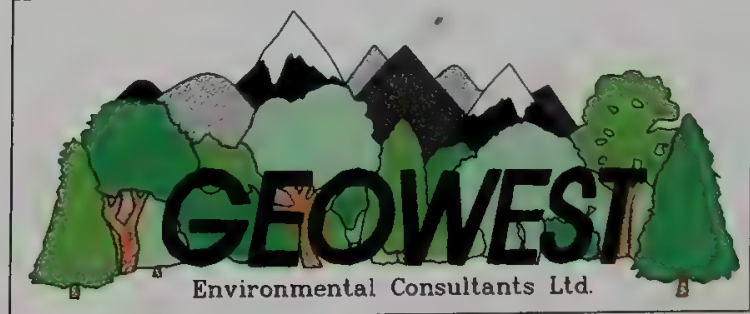

Norember 10, 1998 


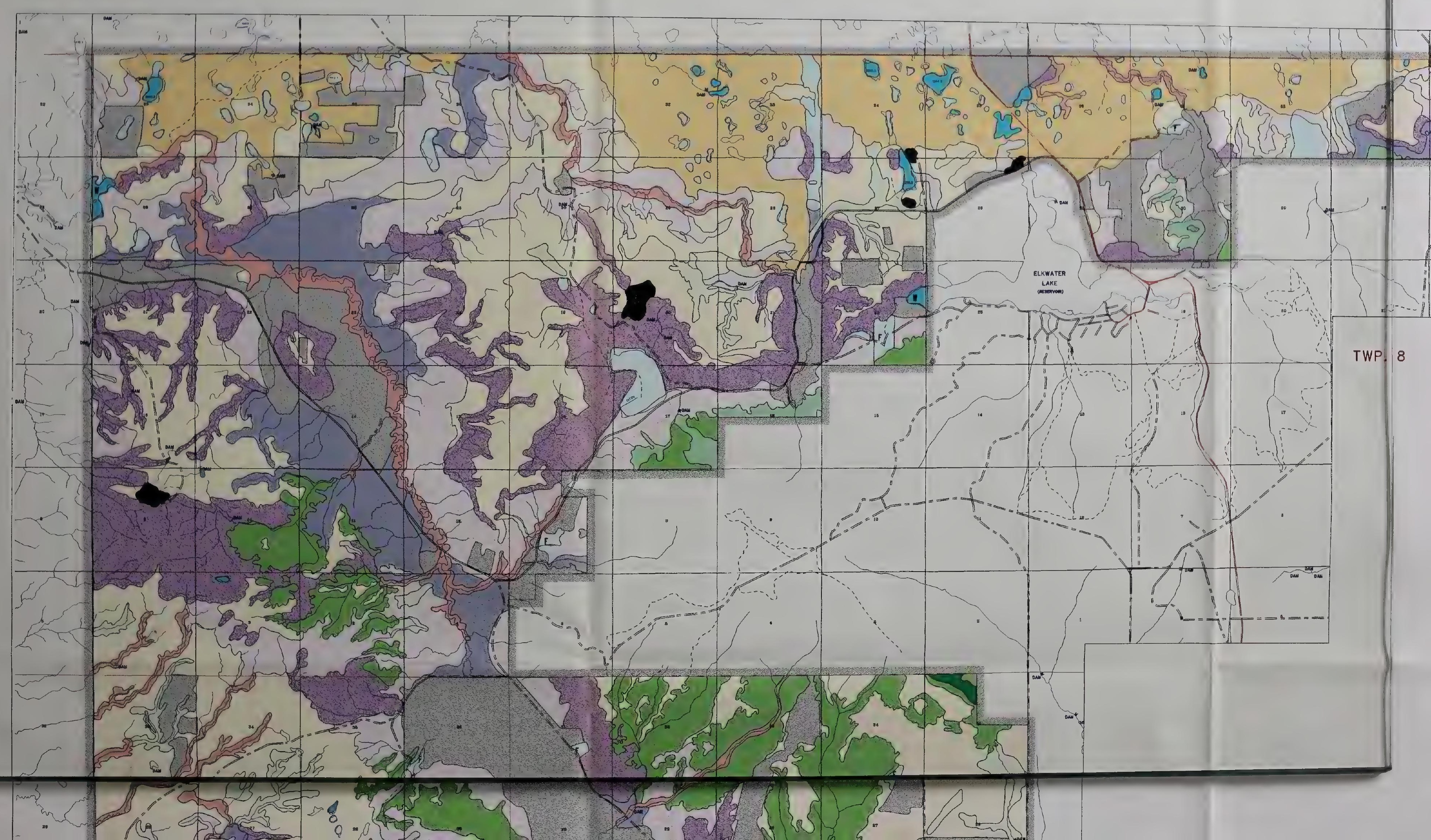





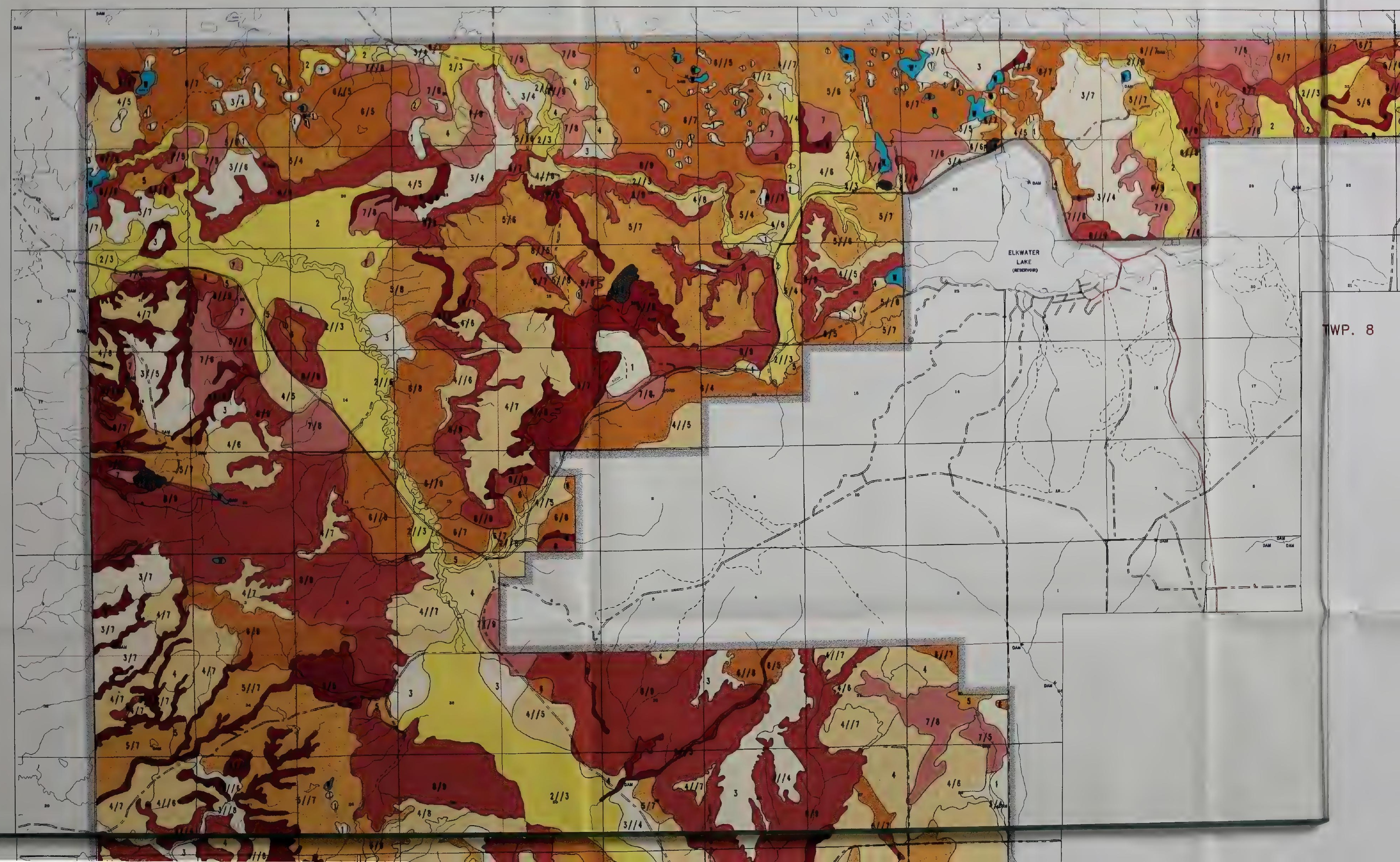




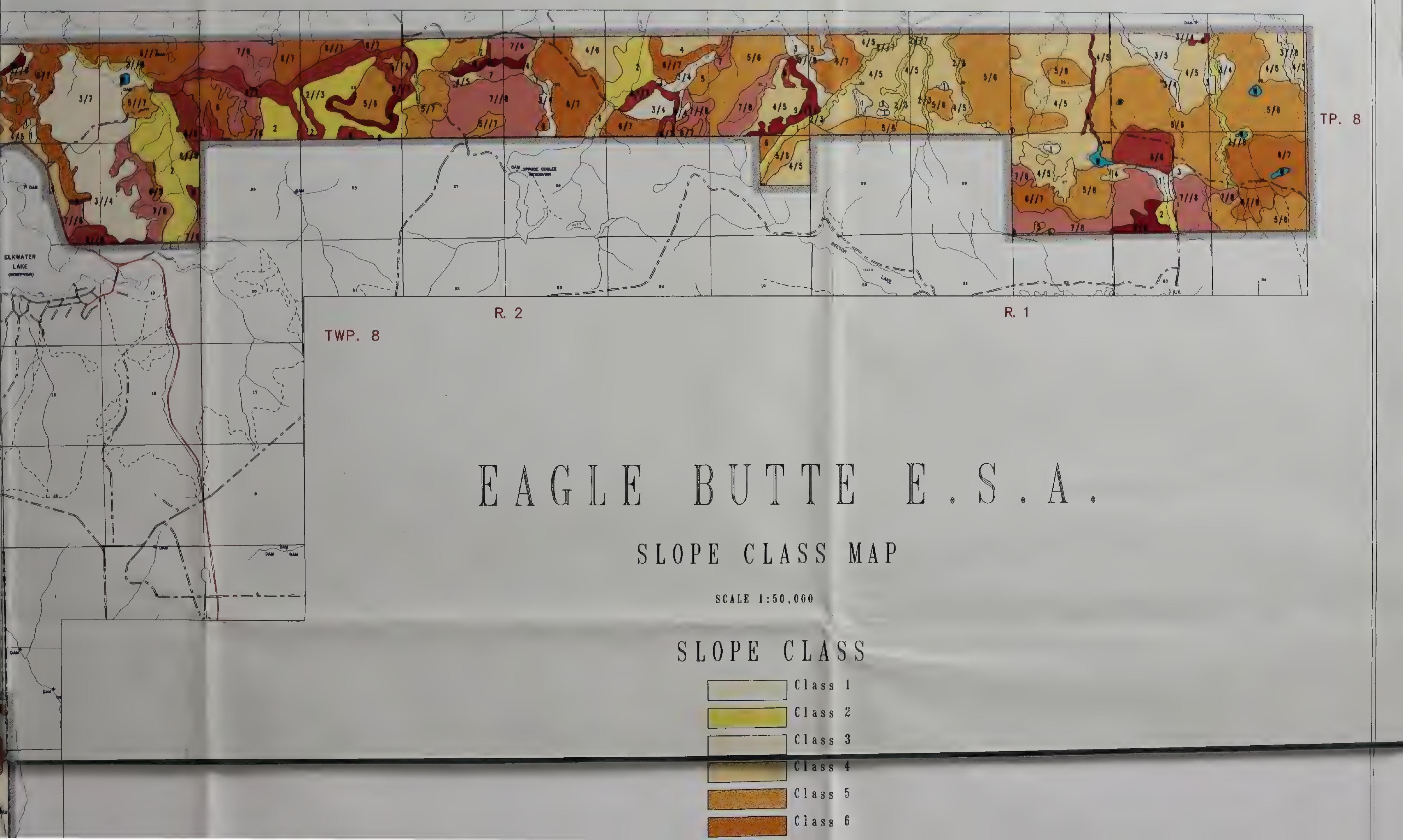




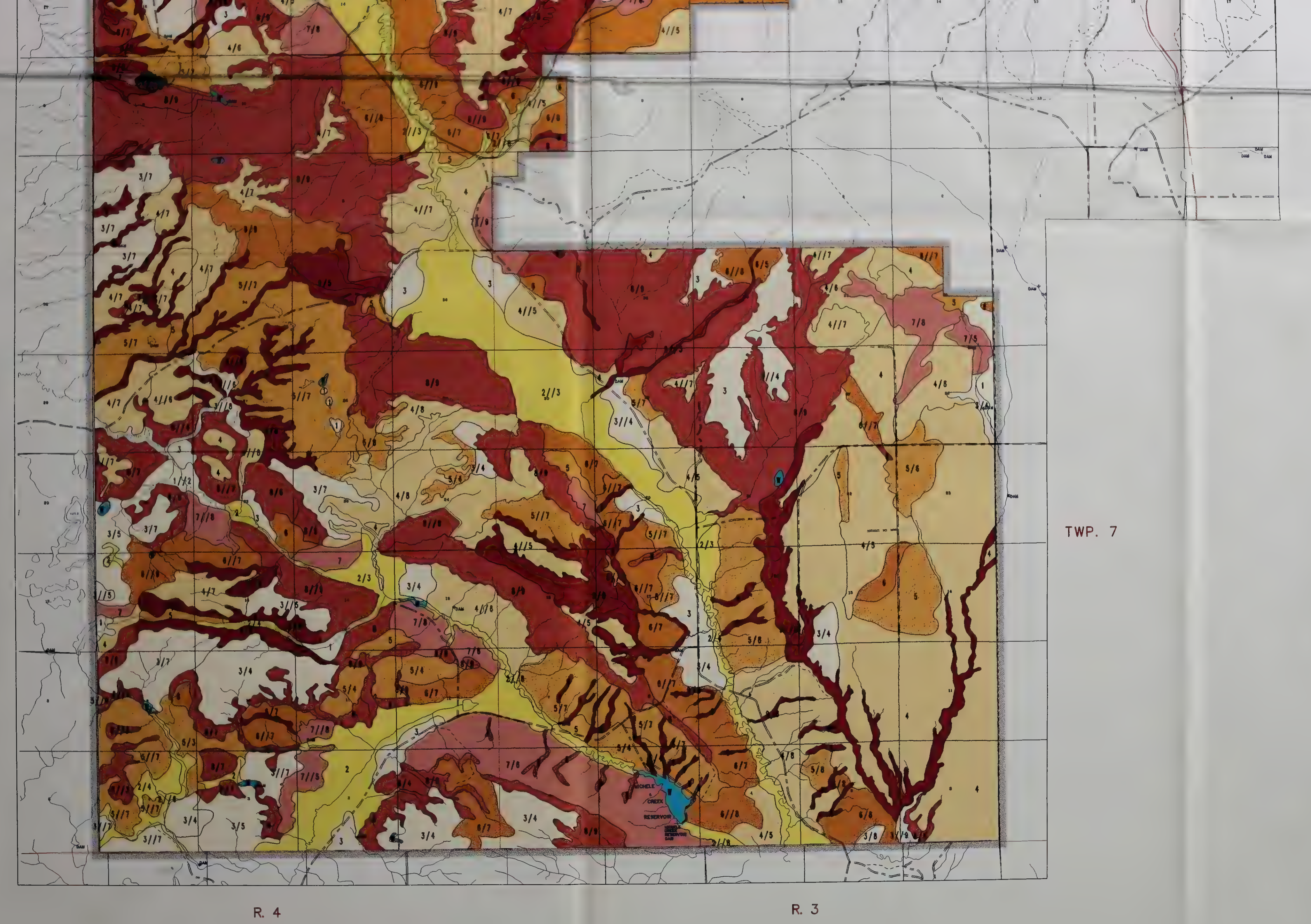




\section{EAGLE BUTTE E.S.A.}

SLOPE CLASS MAP

SCALE $1: 50,000$

\section{SLOPE CLASS}

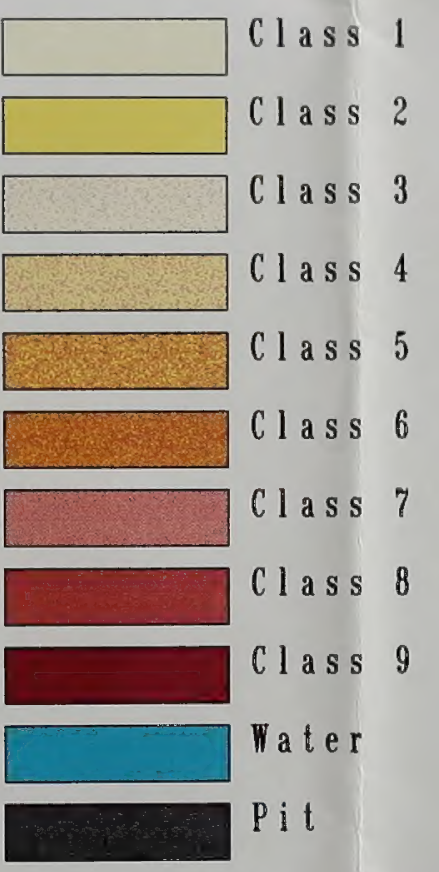

Dominant Slope is Shaded

$4 / 7=60 \%$ of $4,40 \%$ of

$4 / / 7=60 \%$ or

TOPOGRAPHIC BASE FEATURES

Major Highway

Secondary Highway

Roads

Truck Trails

Hydrography

Section Lines 


